\title{
Vector valued spherical functions and Macdonald-Koornwinder polynomials
}

\author{
Alexei A. Oblomkov and Jasper V. Stokman
}

\begin{abstract}
We interpret the five-parameter family of Macdonald-Koornwinder polynomials as vector valued spherical functions on quantum Grassmannians.
\end{abstract}

\section{Introduction}

The representation theoretic construction of (quantum) conformal blocks in certain conformal field theories is closely related to harmonic analysis on (quantum) symmetric spaces of group type, see, e.g., [FR92, EV00]. A striking consequence is the interpretation of $A$-type Macdonald polynomials as vector valued spherical functions for the quantum analogue of the symmetric pair $(\mathrm{U}(n) \times \mathrm{U}(n), \operatorname{diag}(\mathrm{U}(n)))$ of group type on the one hand (see [EK94]), and as quantum conformal blocks on the other hand (see [EV00]). With these interpretations many properties of $A$-type Macdonald polynomials, such as the Macdonald-Ruijsenaars difference equations, quantum KhnizhnikZamolodchikov equations, dualities and orthogonality relations, obtain their natural representation theoretic and conformal field theoretic interpretations. In this paper we consider the harmonic analytic part of these constructions for the quantum analogues of the symmetric pair $(U, K)=$ $(\mathrm{U}(2 n), \mathrm{U}(n) \times \mathrm{U}(n))$. This leads to the interpretation of the five-parameter family of MacdonaldKoornwinder polynomials as vector valued spherical functions.

In [Obl04] the classical analogue of our main result was established. It yields the interpretation of $B C$-type Heckman-Opdam polynomials as the restriction to the maximal torus of regular vector valued functions

$$
f: U \rightarrow \mathbb{C} \operatorname{det}^{-\kappa_{1}} \otimes S^{n \kappa}\left(\mathbb{C}^{n}\right) \operatorname{det}^{\kappa_{1}-\kappa}, \quad \kappa_{1} \in \mathbb{Z}, \kappa \in \mathbb{Z}_{\geqslant 0}
$$

(with $S^{n \kappa}\left(\mathbb{C}^{n}\right)$ the homogeneous polynomials of degree $n \kappa$ ) which transform under the left (respectively right) regular $K$-action on $U$ according to the natural $K$-action on the image space (respectively the $K$-character $\operatorname{det}^{-\kappa_{2}} \otimes \operatorname{det}^{\kappa_{2}}$ for some $\kappa_{2} \in \mathbb{Z}$ ).

In this paper we define a continuous one-parameter family of quantum analogues of the symmetric pair $(U, K)$, following closely Letzter's [Let97, Let99, Let00, Let02, Let03] approach of constructing quantum symmetric pairs as coideal subalgebras. One may as well view this family of quantum symmetric pairs as a continuous one-parameter family of quantum analogues of the complex Grassmannian $U / K$. We proceed by defining the analogue of the $K$-representation $\mathbb{C} \operatorname{det}^{-\kappa_{1}} \otimes S^{n \kappa}\left(\mathbb{C}^{n}\right) \operatorname{det}^{\kappa_{1}-\kappa}$ for the associated coideal subalgebras. This allows us to define vector valued spherical functions for the one-parameter family of quantum symmetric pairs in essentially the same manner as in the classical case [Obl04], with the exception that we have now the additional

Received 18 December 2003, accepted in final form 20 January 2005, published online 1 September 2005.

2000 Mathematics Subject Classification 33D80 (primary), 33D52 (secondary).

Keywords: Macdonald-Koornwinder polynomials, vector valued spherical functions, quantum groups, quantum symmetric pairs.

The second author is supported by the Royal Netherlands Academy of Arts and Sciences (KNAW). The first author is supported by the NSF grant DMS-9988796 and the CRDF grant RM1-2545-MO-03.

This journal is (C) Foundation Compositio Mathematica 2005. 


\section{SPHERICAL FUnCTIONS AND MACDONALD-KOORNWINDER POLYNOMIALS}

freedom to choose different coideal subalgebras for the transformation behaviour under the left and right regular action, respectively. We relate the resulting vector valued spherical functions, which now depend on two continuous and three discrete parameters, to the five-parameter family of Macdonald-Koornwinder polynomials. The Macdonald-Koornwinder polynomials are Koornwinder's [Koo92] extension of the $B C$-type Macdonald polynomials that contain all Macdonald polynomials of classical type as special cases.

The interpretation of the five-parameter family of Macdonald-Koornwinder polynomials as vector valued spherical functions contains several known results as special cases. It entails the interpretation of a two-parameter subfamily of Macdonald-Koornwinder polynomials as zonal spherical functions on quantum Grassmannians, which was established by Noumi et al. [NDS97] (see also [DS99]). In fact, this special case plays an essential role in establishing our general result. In rank one we re-obtain the interpretation of the four-parameter family of Askey-Wilson polynomials as matrix coefficients of quantum sl(2) representations, established before by Koornwinder [Koo93] (zonal case), and by Noumi and Mimachi [NM90] and Koelink [Koe96] (general case).

The content of this paper is as follows. In $\S 1$ we give the main definitions and formulate the main result. In $\S 2$ we define the notion of expectation value for the quantum symmetric pairs under investigation and establish branching rules using deformation theory. In $\S 3$ we prove the zonal case by translating the main results of [NDS97] to our setup. In $\S 4$ we generalize the Chevalley restriction theorem to the setup of vector valued spherical functions. Its description involves the vector valued spherical function of the smallest degree, which we call the ground state (in the zonal case the ground state is the unit). The restriction of the ground state to the quantum torus is computed explicitly in $\S 5$. In $\S 6$ we establish the quantum Schur orthogonality relations for the vector valued spherical functions and, combined with the results of previous sections, we establish the explicit interpretation of the Macdonald-Koornwinder polynomials as vector valued spherical functions.

The construction of expectation values in $\S 2$ and the dynamical quantum group interpretation of the rank one results in [Sto03] hint at a natural interpretation of the Macdonald-Koornwinder polynomials as quantum conformal blocks. A more detailed study in this direction is the subject of future research.

\section{Formulation of the main result}

In this section we give definitions of the Macdonald-Koornwinder polynomials, the quantum symmetric pairs and the associated class of vector valued spherical functions, and we formulate the main result of the paper. We add insightful proofs of some of the intermediate results, but we postpone the more technical parts to later sections. We fix a positive integer $n \geqslant 1$ and a deformation parameter $0<q<1$ throughout the paper.

\subsection{Macdonald-Koornwinder polynomials}

Koornwinder [Koo92] extended the definition of the Macdonald polynomials [Mac00] associated to the non-reduced, irreducible root system $B C_{n}$ to a family of orthogonal polynomials depending on, besides the deformation parameter $q$, five additional coupling parameters. This family of polynomials, known nowadays as Macdonald-Koornwinder polynomials, reduces for $n=1$ to the celebrated four-parameter family of Askey-Wilson polynomials. In this subsection we recall their definition.

Denote by $\Lambda_{n}$ the lattice $\mathbb{Z}^{n}$ and let $\Lambda_{n}^{+} \subset \Lambda_{n}$ be the set of partitions of length $\leqslant n$. The dominance partial order on $\Lambda_{n}$ is defined by

$$
\lambda \leqslant \mu \Longleftrightarrow \sum_{i=1}^{j} \lambda_{i} \leqslant \sum_{i=1}^{j} \mu_{i}, \quad j=1, \ldots, n .
$$




\section{A. A. Oblomkov and J. V. Stokman}

The Weyl group $W=S_{n} \ltimes\{ \pm 1\}^{n}$ of $B C_{n}$, where $S_{n}$ is the symmetric group in $n$ letters, acts on $\Lambda_{n}$ by permutations and sign changes. Each $W$-orbit in $\Lambda_{n}$ intersects $\Lambda_{n}^{+}$exactly once.

Let $\mathbb{C}\left[u^{ \pm 1}\right]=\mathbb{C}\left[u_{1}^{ \pm 1}, \ldots, u_{n}^{ \pm 1}\right]$ be the algebra of Laurent polynomials in $n$ independent variables $u_{k}(1 \leqslant k \leqslant n)$, or, equivalently, the algebra of regular functions on the complex $n$-torus $T^{\mathbb{C}}=\left(\mathbb{C}^{*}\right)^{n}$. A basis of $\mathbb{C}\left[u^{ \pm 1}\right]$ is given by the monomials $u^{\lambda}=u_{1}^{\lambda_{1}} u_{2}^{\lambda_{2}} \cdots u_{n}^{\lambda_{n}}\left(\lambda=\left(\lambda_{1}, \lambda_{2}, \ldots, \lambda_{n}\right) \in \Lambda_{n}\right)$.

The Weyl group $W$ acts on $\mathbb{C}\left[u^{ \pm 1}\right]$ by permutations and inversions of the $u_{k}$. Let $\mathbb{C}\left[u^{ \pm 1}\right]^{W} \subset$ $\mathbb{C}\left[u^{ \pm 1}\right]$ be the subalgebra of $W$-invariant Laurent polynomials. The orbit sums $m_{\lambda}=\sum_{\mu \in W \lambda} u^{\mu}$ $\left(\lambda \in \Lambda_{n}^{+}\right)$form a linear basis of $\mathbb{C}\left[u^{ \pm 1}\right]^{W}$.

The Macdonald-Koornwinder polynomials form an orthogonal basis of $\mathbb{C}\left[u^{ \pm 1}\right]^{W}$ with respect to a particular scalar product on $\mathbb{C}\left[u^{ \pm 1}\right]^{W}$. The scalar product, which we now define first, depends on five additional coupling parameters $a, b, c, d$ and $t$. It is defined in terms of an absolutely continuous measure with respect to the normalized Haar measure on the natural compact real form $T=\mathbb{T}^{n}$ of $T^{\mathbb{C}}=\left(\mathbb{C}^{*}\right)^{n}$, where $\mathbb{T}$ is the unit circle in the complex plane. The corresponding weight function $\Delta$ is most conveniently expressed in terms of the $q$-shifted factorial,

$$
(a ; q)_{k}=\prod_{i=0}^{k-1}\left(1-a q^{i}\right), \quad \forall k \in \mathbb{Z}_{+} \cup\{\infty\}
$$

by $\Delta(u)=\Delta^{+}(u) \Delta^{+}\left(u^{-1}\right)$ with $\Delta^{+}(u)=\Delta^{+}(u ; a, b, c, d ; q, t)$ defined by

$$
\Delta^{+}(u)=\prod_{i=1}^{n} \frac{\left(u_{i}^{2} ; q\right)_{\infty}}{\left(a u_{i}, b u_{i}, c u_{i}, d u_{i} ; q\right)_{\infty}} \prod_{1 \leqslant i<j \leqslant n} \frac{\left(u_{i} / u_{j}, u_{i} u_{j} ; q\right)_{\infty}}{\left(t u_{i} / u_{j}, t u_{i} u_{j} ; q\right)_{\infty}} .
$$

Here $\left(a_{1}, \ldots, a_{s} ; q\right)_{k}=\prod_{j=1}^{s}\left(a_{j} ; q\right)_{k}$ is a short-hand notation for products of $q$-shifted factorials. If $a, b, c, d, t$ are real and

$$
|a|,|b|,|c|,|d|<1, \quad 0<t<1,
$$

then $\Delta(u)$ is a positive continuous weight function on $T$. Under these assumptions, we can define the Macdonald-Koornwinder polynomials as follows, see [Koo92].

Theorem 1.1. There exist unique $W$-invariant Laurent polynomials

$$
P_{\lambda}(u)=P_{\lambda}(u ; a, b, c, d ; q, t) \in \mathbb{C}\left[u^{ \pm 1}\right]^{W}, \quad \lambda \in \Lambda_{n}^{+}
$$

satisfying the two conditions

$$
\begin{gathered}
P_{\lambda}(u)=m_{\lambda}+\sum_{\mu \in \Lambda_{n}^{+}: \mu<\lambda} c_{\lambda \mu} m_{\mu}, \quad \text { some } c_{\lambda \mu} \in \mathbb{C}, \\
\int_{T} P_{\lambda}(u) \overline{P_{\mu}(u)} \Delta(u) \frac{d u}{u}=0, \quad \text { if } \lambda \neq \mu,
\end{gathered}
$$

where

$$
\frac{d u}{u}=\frac{d u_{1}}{u_{1}} \frac{d u_{2}}{u_{2}} \cdots \frac{d u_{n}}{u_{n}}
$$

is the Haar measure on $T$. We call $P_{\lambda}(u)$ the monic Macdonald-Koornwinder polynomial of degree $\lambda \in \Lambda_{n}^{+}$.

The theorem does not follow by a straightforward Gram-Schmidt type procedure since the dominance ordering is not a total ordering. The key tool in proving the theorem is an explicit self-adjoint difference operator which maps a symmetric monomial $m_{\lambda}$ to a linear combination of symmetric monomials $m_{\mu}$ involving only degrees $\mu \leqslant \lambda$ (see [Koo92]). In our setup, the self-adjoint operator arises as the radial part of the quadratic Casimir element for the corresponding quantum symmetric pair (see [NDS97, Theorem 3.3]). 


\section{SPHERICAL FUnCTIONS AND MACDONALD-KOORNWINDER POLYNOMIALS}

Remark 1.2. Theorem 1.1 is also valid for generic values of the parameters $a, b, c$ and $d$ outside the region (1.1) after deforming the compact torus $T$ in the definition of the orthogonality relations in a suitable way. In general, one loses positivity of the weight function, but for suitable values of the parameters (still violating the condition that the modulus of all the four parameters $a, b, c$ and $d$ is less than one), one can re-obtain a positive measure by shifting the deformed compact torus to $T$ while picking up residues, see [Sto00].

\subsection{The quantized universal enveloping algebra}

We introduce here the notation for the quantized universal enveloping algebra of $\mathfrak{g l}(m)$. For further details and standard facts, we refer to [NYM93] and [Nou96].

In the notation below we suppress the dependence on $m \in \mathbb{Z}_{>0}$ as much as possible. For most of our applications, $m$ will be either $2 n$ (in which case we write $\mathfrak{g}$ for the Lie algebra $\mathfrak{g l}(2 n)$ ) or $m$ will be $n$ (in which case we stick to the notation $\mathfrak{g l}(n))$. Let $\delta_{i, j}$ be the usual Kronecker delta function ( $=1$ if $i=j$, and $=0$ otherwise). The quantized universal enveloping algebra $U_{q}(\mathfrak{g l}(m))$ is the unital algebra over $\mathbb{C}$ generated by $K_{i}^{ \pm 1}(i=1, \ldots, m), x_{j}, y_{j}(j=1, \ldots, m-1)$, subject to the relations

$$
\begin{gathered}
K_{i} K_{j}=K_{j} K_{j}, \quad K_{i} K_{i}^{-1}=1=K_{i}^{-1} K_{i}, \\
K_{i} x_{j} K_{i}^{-1}=q^{\delta_{i, j}-\delta_{i, j+1}} x_{j}, \quad K_{i} y_{j} K_{i}^{-1}=q^{-\delta_{i, j}+\delta_{i, j+1}} y_{j}, \\
x_{i} y_{j}-y_{j} x_{i}=\frac{K_{i} K_{i+1}^{-1}-K_{i}^{-1} K_{i+1}}{q-q^{-1}} \delta_{i, j}, \\
x_{i} x_{j}=x_{j} x_{i} \quad \text { and } \quad y_{i} y_{j}=y_{j} y_{i}, \quad \text { when }|i-j| \geqslant 2, \\
x_{i}^{2} x_{j}-\left(q+q^{-1}\right) x_{i} x_{j} x_{i}+x_{j} x_{i}^{2}=0, \quad \text { when }|i-j|=1, \\
y_{i}^{2} y_{j}-\left(q+q^{-1}\right) y_{i} y_{j} y_{i}+y_{j} y_{i}^{2}=0, \quad \text { when }|i-j|=1 .
\end{gathered}
$$

The quantized universal enveloping algebra $U_{q}(\mathfrak{g l}(m))$ is a Hopf $*$-algebra, with co-multiplication

$$
\begin{aligned}
\Delta\left(x_{j}\right) & =x_{j} \otimes 1+K_{j} K_{j+1}^{-1} \otimes x_{j}, \\
\Delta\left(y_{j}\right) & =y_{j} \otimes K_{j}^{-1} K_{j+1}+1 \otimes y_{j}, \\
\Delta\left(K_{i}^{ \pm 1}\right) & =K_{i}^{ \pm 1} \otimes K_{i}^{ \pm 1},
\end{aligned}
$$

co-unit

antipode

$$
\varepsilon\left(x_{j}\right)=\varepsilon\left(y_{j}\right)=0, \quad \varepsilon\left(K_{i}^{ \pm 1}\right)=1,
$$

$$
S\left(x_{j}\right)=-K_{j}^{-1} K_{j+1} x_{j}, \quad S\left(y_{j}\right)=-y_{j} K_{j} K_{j+1}^{-1}, \quad S\left(K_{i}^{ \pm 1}\right)=K_{i}^{\mp 1}
$$

and $*$-structure

$$
x_{j}^{*}=q^{-1} y_{j} K_{j} K_{j+1}^{-1}, \quad y_{j}^{*}=q K_{j}^{-1} K_{j+1} x_{j}, \quad\left(K_{i}^{ \pm 1}\right)^{*}=K_{i}^{ \pm 1} .
$$

This $*$-structure corresponds classically to choosing the skew-hermitean matrices $\mathfrak{u}(m)$ as the (compact) real form of $\mathfrak{g l}(m)$. We use the (modified) Sweedler notation $\Delta(X)=\sum X_{1} \otimes X_{2}\left(X \in U_{q}(\mathfrak{g})\right)$ for the co-multiplication.

\subsection{The quantum analogues of the symmetric pair $(\mathfrak{g l}(2 n), \mathfrak{g l}(n) \times \mathfrak{g l}(n))$}

The realization of a two-parameter subfamily of the Macdonald-Koornwinder polynomials as zonal spherical functions on quantizations of the complex Grassmannian by Noumi et al. [NDS97], see also [DS99], plays a crucial role in the present paper. A one-parameter family of quantum Grassmannians is defined in [NDS97] in terms of invariance properties with respect to a one-parameter family of 


\section{A. A. Oblomkov and J. V. Stokman}

two-sided coideals, which are analogues of the Lie subalgebra $\mathfrak{k}=\mathfrak{g l}(n) \times \mathfrak{g l}(n) \subset \mathfrak{g}=\mathfrak{g l}(2 n)$. Although there is a natural way to define the analogue of the trivial representation of $\mathfrak{k}$ to the coideal setup, this is no longer the case for other representations. To get a grip on the non-trivial representations, we replace the two-sided coideals by coideal subalgebras of $U_{q}(\mathfrak{g})$, an idea which was suggested by Noumi [Nou96] and developed in full generality in a series of papers [Let97, Let99, Let00, Let02, Let03, Let04] by Letzter. For the quantum symmetric pair $(\mathfrak{g}, \mathfrak{k})=(\mathfrak{g l} l(2 n), \mathfrak{g l}(n) \times$ $\mathfrak{g l}(n))$ the coideal algebras have a particularly nice presentation in terms of generators and relations, closely resembling the relations of the Drinfeld-Jimbo quantized universal enveloping algebras (see [Let99, Let03]).

One of the more technical parts of this paper is the translation of the results of Noumi et al. [NDS97] into the language of right coideal algebras. Unfortunately, in doing so it turns out to be more convenient to work with a slightly modified version of Letzter's right coideal algebra. In this subsection we define the modified right coideal algebra for the symmetric pair $(\mathfrak{g}, \mathfrak{k})$, and we present some of its important properties.

Let $\left\{\epsilon_{i}\right\}_{i=1}^{2 n}$ be the standard orthonormal basis of the Euclidean space $\left(\mathbb{R}^{2 n},(\cdot, \cdot)\right)$ and denote

$$
\alpha_{j}=\epsilon_{j}-\epsilon_{j+1}, \quad j=1, \ldots, 2 n-1 .
$$

Definition 1.3. Let $\mathcal{A}$ be the unital, associative algebra over $\mathbb{C}$ with generators $\gamma_{i}^{ \pm 1}(i=1, \ldots, 2 n)$ and $\beta_{j}(j=1, \ldots, 2 n-1)$ satisfying the relations:

$$
\gamma_{i} \gamma_{j}=\gamma_{j} \gamma_{i}, \quad \gamma_{i} \gamma_{i}^{-1}=1=\gamma_{i}^{-1} \gamma_{i}, \quad \gamma_{i}=\gamma_{2 n+1-i}
$$

for $i, j=1, \ldots, 2 n$;

$$
\gamma_{i} \beta_{j}=q^{-\left(\epsilon_{i}+\epsilon_{2 n+1-i}, \alpha_{j}\right)} \beta_{j} \gamma_{i}
$$

for $i=1, \ldots, 2 n$ and $j=1, \ldots, 2 n-1$;

$$
\beta_{i} \beta_{j}-\beta_{j} \beta_{i}=q\left(\frac{\gamma_{i}^{-2}-\gamma_{i+1}^{-2}}{q-q^{-1}}\right) \delta_{i, 2 n-j}
$$

for $i, j=1, \ldots, 2 n-1$ with $|i-j| \geqslant 2$;

$$
q^{-1} \beta_{i}^{2} \beta_{i+1}-\left(q+q^{-1}\right) \beta_{i} \beta_{i+1} \beta_{i}+q \beta_{i+1} \beta_{i}^{2}=q \beta_{n+1} \gamma_{n}^{-2} \delta_{i, n}
$$

for $i=1, \ldots, 2 n-2$ and

$$
q \beta_{i}^{2} \beta_{i-1}-\left(q+q^{-1}\right) \beta_{i} \beta_{i-1} \beta_{i}+q^{-1} \beta_{i-1} \beta_{i}^{2}=q^{-1} \beta_{n-1} \gamma_{n}^{-2} \delta_{i, n}
$$

for $i=2, \ldots, 2 n-1$.

Observe that $\mathcal{A}$ becomes a $*$-algebra with $*$-structure defined by

$$
\gamma_{i}^{*}=\gamma_{i}, \quad \beta_{j}^{*}=\beta_{2 n-j}
$$

for $i=1, \ldots, 2 n$ and $j=1, \ldots, 2 n-1$.

Proposition 1.4. Let $\sigma \in \mathbb{R}$. The assignment

$$
\begin{aligned}
\pi_{\sigma}\left(\gamma_{i}^{ \pm 1}\right) & =K_{i}^{ \pm 1} K_{2 n+1-i}^{ \pm 1}, \\
\pi_{\sigma}\left(\beta_{j}\right) & =y_{j} K_{j+1}^{-1} K_{2 n-j}^{-1}+K_{j}^{-1} x_{2 n-j} K_{2 n-j}^{-1}, \\
\pi_{\sigma}\left(\beta_{n}\right) & =y_{n} K_{n+1}^{-1} K_{n}^{-1}+K_{n}^{-1} x_{n} K_{n}^{-1}+\left(\frac{q^{-\sigma}-q^{\sigma}}{q-q^{-1}}\right) K_{n}^{-2}
\end{aligned}
$$

for $i \in\{1, \ldots, 2 n\}$ and $j \in\{1, \ldots, 2 n-1\} \backslash\{n\}$ uniquely extends to an injective $*$-algebra homomorphism $\pi_{\sigma}: \mathcal{A} \rightarrow U_{q}(\mathfrak{g})$. 


\section{SPHERICAL FUnCTIONS AND MACDONALD-KOORNWINDER POLYNOMIALS}

Proof. This is essentially [Let03, Theorem 7.1] for the symmetric pair of type AIII (Case 2), see $[$ Let03, $\S 7]$. More specifically, following the proof of [Let97, Lemma 2.2] one shows that the assignment (1.8) uniquely extends to a unital $*$-algebra homomorphism $\pi_{\sigma}: \mathcal{A} \rightarrow U_{q}(\mathfrak{g})$ for $\sigma \in \mathbb{R}$. The injectivity of $\pi_{\sigma}$ follows by a straightforward modification of the proof of [Let97, Proposition 2.3].

Definition 1.5. Let $\sigma \in \mathbb{R}$. We call $\left(U_{q}(\mathfrak{g}), \mathcal{A}_{\sigma}\right)$ with $\mathcal{A}_{\sigma}=\pi_{\sigma}(\mathcal{A})$ a quantum analogue of the symmetric pair $(\mathfrak{g}, \mathfrak{k})=(\mathfrak{g l}(2 n), \mathfrak{g l}(n) \times \mathfrak{g l}(n))$. We denote

$$
C_{i}=\pi_{\sigma}\left(\gamma_{i}\right), \quad B_{j}=\pi_{\sigma}\left(\beta_{j}\right), \quad B_{n}^{\sigma}=\pi_{\sigma}\left(\beta_{n}\right)
$$

$(i \in\{1, \ldots, 2 n\}$ and $j \in\{1, \ldots, 2 n-1\} \backslash\{n\})$ for the image of the generators of $\mathcal{A}$ under the embedding $\pi_{\sigma}$.

We fix $\sigma \in \mathbb{R}$ once and for all, unless specified differently.

The terminology in Definition 1.5 can formally be justified as follows, see e.g., [Let97, § 3]. Taking the classical limit $q \rightarrow 1$ of the (modified) generators of $\mathcal{A}_{\sigma}$ gives

$$
\begin{gathered}
\frac{C_{i}-C_{i}^{-1}}{q-q^{-1}} \rightarrow E_{i, i}+E_{2 n+1-i, 2 n+1-i}, \\
B_{j} \rightarrow E_{j+1, j}+E_{2 n-j, 2 n+1-j} \\
B_{n}^{\sigma} \rightarrow E_{n+1, n}+E_{n, n+1}-\sigma 1
\end{gathered}
$$

for $i \in\{1, \ldots, n\}$ and $j \in\{1, \ldots, 2 n-1\} \backslash\{n\}$, where $E_{i, j} \in \mathfrak{g} \subset U(\mathfrak{g})$ is the matrix unit with a one in row $i$ and column $j$, and zeros elsewhere. The unital subalgebra $\widetilde{\mathcal{A}}$ of $U(\mathfrak{g})$ generated by the classical limits (1.10) of the generators of $\mathcal{A}_{\sigma}$ is independent of $\sigma$ and isomorphic to the subalgebra $U(\mathfrak{k}) \subset U(\mathfrak{g})$. The isomorphism is induced by the inner automorphism $\operatorname{Ad}(g)$ of $\mathfrak{g}$, with $g \in \mathrm{GL}(2 n ; \mathbb{C})$ given explicitly by

$$
g=\frac{1}{\sqrt{2}} \sum_{i=1}^{n}\left(E_{i, i}+E_{i, 2 n+1-i}+E_{2 n+1-i, i}-E_{2 n+1-i, 2 n+1-i}\right) .
$$

In fact, the associated inner automorphism of $U(\mathfrak{g})$ preserves all classical generators (1.10) of $\widetilde{\mathcal{A}}$ besides $E_{n+1, n}+E_{n, n+1}-\sigma 1$, which is mapped to $E_{n, n}-E_{n+1, n+1}-\sigma 1$.

The classical isomorphism $\widetilde{\mathcal{A}} \simeq U(\mathfrak{k})$ via the inner automorphism $\operatorname{Ad}(g)$ has the following weak analogue on the quantum group level. Let $U_{q}(\mathfrak{k})$ be the Hopf $*$-subalgebra of $U_{q}(\mathfrak{g})$ generated by $K_{i}^{ \pm 1}(i \in\{1, \ldots, 2 n\})$ and $x_{j}, y_{j}(j \in\{1, \ldots, 2 n-1\} \backslash\{n\})$. Denote

$$
\widehat{B}_{n}^{\sigma}=\frac{q^{-\sigma} K_{n+1}^{-2}-q^{\sigma} K_{n}^{-2}}{q-q^{-1}} \in U_{q}(\mathfrak{k}),
$$

which has $E_{n, n}-E_{n+1, n+1}-\sigma 1$ as classical limit.

Proposition 1.6. Let $I \subseteq U_{q}(\mathfrak{k})$ be the two-sided $*$-ideal generated by $x_{n-1} y_{n+1}$. The assignment

$$
\phi_{\sigma}\left(\gamma_{i}\right)=C_{i}+I, \quad \phi_{\sigma}\left(\beta_{j}\right)=B_{j}+I, \quad \phi_{\sigma}\left(\beta_{n}\right)=\widehat{B}_{n}^{\sigma}+I
$$

for $i \in\{1, \ldots, n\}$ and $j \in\{1, \ldots, 2 n-1\} \backslash\{n\}$ uniquely extends to a $*$-algebra homomorphism $\phi_{\sigma}: \mathcal{A} \rightarrow U_{q}(\mathfrak{k}) / I$.

Proof. In view of Proposition 1.4, $\phi_{\sigma}$ extends to an algebra homomorphism $\phi_{\sigma}: \mathcal{A} \rightarrow U_{q}(\mathfrak{k}) / I$ if $\phi_{\sigma}$ respects the defining relations of $\mathcal{A}$ involving $\beta_{n}$. This is a straightforward, but tedious computation. The $*$-ideal $I$ only plays a role for the relations (1.6) for $i=n-1$ and relation (1.7) for $i=n+1$ (which in turn are each others $*$-images). In $U_{q}(\mathfrak{k})$ we have the relation

$$
q^{-1} B_{n-1}^{2} \widehat{B}_{n}^{\sigma}-\left(q+q^{-1}\right) B_{n-1} \widehat{B}_{n}^{\sigma} B_{n-1}+q \widehat{B}_{n}^{\sigma} B_{n-1}^{2}=q^{-\sigma-1}\left(q^{-2}-q^{2}\right) x_{n+1} y_{n-1} K_{n-1}^{-1} K_{n}^{-1} K_{n+1}^{-4},
$$




\section{A. A. Oblomkov and J. V. Stokman}

which, modulo $I$, reduces to the $\phi_{\sigma}$-image of relation (1.6) for $i=n-1$. The case $i=n+1$ is checked similarly. It is clear that the algebra homomorphism $\phi_{\sigma}$ respects the $*$-structure.

Remark 1.7. Denote $U_{q}(\mathfrak{g l}(2))^{(n)} \subset U_{q}(\mathfrak{g})$ for the copy of the unital Hopf-*-algebra $U_{q}(\mathfrak{g l}(2))$ in $U_{q}(\mathfrak{g})$, generated by $x_{n}, y_{n}, K_{n}^{ \pm 1}, K_{n+1}^{ \pm 1}$. Note that both $B_{n}^{\sigma} \in U_{q}(\mathfrak{g l}(2))^{(n)}$ and $\widehat{B}_{n}^{\sigma} \in U_{q}(\mathfrak{g l}(2))^{(n)}$, with $\widehat{B}_{n}^{\sigma}$ given by (1.12). By [Ros00], there exists an invertible element $x_{\sigma}$ in a suitable completion of $U_{q}(\mathfrak{g l}(2))^{(n)}$ such that $x_{\sigma} B_{n}^{\sigma} x_{\sigma}^{-1}=\widehat{B}_{n}^{\sigma}$. Explicitly, $x_{\sigma}$ can be given as an formal infinite series by

$$
x_{\sigma}=\sum_{l, m=0}^{\infty} \frac{q^{-(l+m) \sigma}(-1)^{m}}{\left(-q^{2-2 \sigma} ; q^{2}\right)_{l}} \frac{q^{l^{2}+2 l m-l-m}}{\left(q^{2} ; q^{2}\right)_{l}\left(q^{2} ; q^{2}\right)_{m}}\left(1-q^{2}\right)^{l+m} x_{n}^{l}\left(y_{n} K_{n} K_{n+1}^{-1}\right)^{m} .
$$

The element $x_{\sigma}$ is essentially Babelon's [Bab91] vertex-IRF transformation, which leads to an interpretation of the parameter $\sigma$ as a dynamical parameter in the sense of dynamical quantum groups (see [Sto03] for a detailed discussion and further references). Note, furthermore, that conjugating by $x_{\sigma}$ fixes all other generators $B_{j}$ and $C_{i}^{ \pm 1}$ of $\mathcal{A}_{\sigma}$ besides $B_{n-1}$ and $B_{n+1}$.

Let $\mathcal{E} \subset \mathcal{A}_{\sigma} \subset U_{q}(\mathfrak{g})$ be the unital subalgebra generated by the $\sigma$-independent generators $C_{i}^{ \pm 1}$ $(i \in\{1, \ldots, 2 n\})$ and $B_{j}(j \in\{1, \ldots, 2 n-1\} \backslash\{n\})$ of $\mathcal{A}_{\sigma}$. We end this subsection by proving that $\mathcal{E}$ is essentially the subalgebra $U_{q}(\mathfrak{g l}(n))$, diagonally embedded in $U_{q}(\mathfrak{g})$.

Throughout this paper we identify

$$
U_{q}(\mathfrak{g l}(n)) \otimes U_{q}(\mathfrak{g l}(n)) \simeq U_{q}(\mathfrak{k}) \subset U_{q}(\mathfrak{g})
$$

as $*$-Hopf algebras, by identifying $x_{j} \otimes 1, y_{j} \otimes 1$ and $K_{i}^{ \pm 1} \otimes 1$ (respectively $1 \otimes x_{j}, 1 \otimes y_{j}$ and $1 \otimes K_{i}^{ \pm 1}$ ) with $x_{j}, y_{j}$ and $K_{i}^{ \pm 1}$ (respectively $x_{n+j}, y_{n+j}$ and $K_{n+i}^{ \pm 1}$ ) for $j=1, \ldots, n-1$ and $i=1, \ldots, n$. Let $\psi: U_{q}(\mathfrak{g l}(n)) \rightarrow U_{q}(\mathfrak{g l}(n))$ be the $*$-algebra isomorphism defined by

$$
\psi\left(x_{j}\right)=y_{n-j}, \quad \psi\left(y_{j}\right)=x_{n-j}, \quad \psi\left(K_{i}^{ \pm 1}\right)=K_{n+1-i}^{ \pm 1}
$$

for $j=1, \ldots, n-1$ and $i=1, \ldots, n$. Let $\Delta^{\mathrm{op}}$ be the opposite comultiplication of $U_{q}(\mathfrak{g l}(n))$.

Lemma 1.8. For $j=1, \ldots, n-1$ and $i=1, \ldots, n$ we have

$$
\begin{gathered}
(\mathrm{id} \otimes \psi) \Delta^{\mathrm{op}}\left(y_{j} K_{j+1}^{-1}\right)=B_{j}, \\
(\mathrm{id} \otimes \psi) \Delta^{\mathrm{op}}\left(x_{j} K_{j}^{-1}\right)=B_{2 n-j}, \\
(\mathrm{id} \otimes \psi) \Delta^{\mathrm{op}}\left(K_{i}^{ \pm 1}\right)=C_{i}^{ \pm 1} .
\end{gathered}
$$

Furthermore, $(\mathrm{id} \otimes \psi) \Delta^{\mathrm{op}}$ defines a $*$-algebra isomorphism

$$
(\mathrm{id} \otimes \psi) \Delta^{\mathrm{op}}: U_{q}(\mathfrak{g l}(n)) \stackrel{\sim}{\longrightarrow} \mathcal{E} \subseteq \mathcal{A}_{\sigma} \cap U_{q}(\mathfrak{k}) \subset U_{q}(\mathfrak{g}) .
$$

Proof. The proof is straightforward.

Other crucial properties of the quantum symmetric pairs $\left(U_{q}(\mathfrak{g}), \mathcal{A}_{\sigma}\right)$, such as the coideal property of $\mathcal{A}_{\sigma} \subset U_{q}(\mathfrak{g})$, are discussed in later sections.

\subsection{Representations}

We discuss those aspects of the representation theory associated to the quantum symmetric pairs $\left(U_{q}(\mathfrak{g}), \mathcal{A}_{\sigma}\right)$ which are relevant for the purposes of this paper. We start by recalling some standard facts on the finite-dimensional representation theory of the quantized universal enveloping algebra $U_{q}(\mathfrak{g l}(m))$. If no confusion can arise, we surpress in the following definitions the dependence on $m$ as much as possible.

Let $P_{m}=\bigoplus_{i=1}^{m} \mathbb{Z} \varepsilon_{i} \simeq \mathbb{Z}^{\times m}$ be the rational character lattice of $\operatorname{GL}(m ; \mathbb{C})$ and

$$
P_{m}^{+}=\left\{\left(\lambda_{1}, \ldots, \lambda_{m}\right) \in P_{m} \mid \lambda_{1} \geqslant \cdots \geqslant \lambda_{m}\right\}
$$

the associated cone of dominant weights. 


\section{SPHERICAL FUnCTIONS AND MACDONALD-KOORNWINDER POLYNOMIALS}

We only consider finite-dimensional left $U_{q}(\mathfrak{g l}(m))$-modules $M$ with weights in $P_{m}$, i.e. we assume that $M$ has weight decomposition

$$
\begin{aligned}
M & =\bigoplus_{\mu \in P_{m}} M[\mu], \\
M[\mu] & =\left\{v \in M \mid K_{i} v=q^{\mu_{i}} v, i=1, \ldots, m\right\} .
\end{aligned}
$$

Any such finite-dimensional $U_{q}(\mathfrak{g})$-module $M$ is $U_{q}(\mathfrak{g})$-semisimple. The irreducible ones are the irreducible finite-dimensional highest weight representations $L_{\lambda}$ of $U_{q}(\mathfrak{g l}(m))$ with highest weight $\lambda \in P_{m}^{+}$.

The irreducible module $L_{\lambda}\left(\lambda \in P_{m}^{+}\right)$has a one-dimensional weight space $L_{\lambda}[\lambda]$. A vector $v_{\lambda}$ spanning $L_{\lambda}[\lambda]$ is called a highest weight vector of $L_{\lambda}$. A highest weight vector $v_{\lambda}$ is a cyclic vector of $L_{\lambda}$ satisfying $x_{j} v_{\lambda}=0$ for $j=1, \ldots, m-1$. In particular,

$$
L_{\lambda}=\bigoplus_{\mu \in P_{m}: \mu \preceq \lambda} L_{\lambda}[\mu]
$$

with $\preceq$ the $A$-type dominance order on $P_{m}: \lambda \preceq \mu$ for $\lambda, \mu \in P_{m}$ if

$$
\sum_{i=1}^{j} \lambda_{i} \leqslant \sum_{i=1}^{j} \mu_{i} \quad(j \in\{1, \ldots, m-1\}), \quad \sum_{i=1}^{m} \lambda_{i}=\sum_{i=1}^{m} \mu_{i} .
$$

There exists a scalar product $\langle\cdot, \cdot\rangle_{\lambda}$ on $L_{\lambda}$, unique up to constant multiples, such that

$$
\langle X v, w\rangle_{\lambda}=\left\langle v, X^{*} w\right\rangle_{\lambda}, \quad \forall v, w \in L_{\lambda}, \forall X \in U_{q}(\mathfrak{g l}(m)) .
$$

Consequently, any finite-dimensional $U_{q}(\mathfrak{g l}(m))$-module $M$ is $*$-unitarizable.

Definition 1.9. We write $M^{\sigma}$ for a left $U_{q}(\mathfrak{g})$-module $M$, viewed as a $\mathcal{A}_{\sigma}$-module by restriction of the action to the subalgebra $\mathcal{A}_{\sigma} \subset U_{q}(\mathfrak{g})$.

Since $\mathcal{A}_{\sigma} \subset U_{q}(\mathfrak{g})$ is $*$-invariant, we have the following result, see e.g., [Let00, Theorem 3.3].

Lemma 1.10. If $M$ is a finite-dimensional $U_{q}(\mathfrak{g})$-module, then $M^{\sigma}$ is $\mathcal{A}_{\sigma}$-semisimple.

An abstract representation theory for $\mathcal{A}_{\sigma}$ was developed by Letzter [Let00, $\left.\S \S 5-7\right]$ in the general context of quantum symmetric pairs. For our purposes it is more convenient to have concrete realizations of certain special $\mathcal{A}_{\sigma}$-representations. These special representations are constructed using the following corollary of Proposition 1.6.

Corollary 1.11. Let $V$ be a finite-dimensional Hilbert space and let $\rho: U_{q}(\mathfrak{k}) \rightarrow \operatorname{End}_{\mathbb{C}}(V)$ be a $*$-representation such that $I \subseteq \operatorname{Ker}(\rho)$. Denote by $\bar{\rho}: U_{q}(\mathfrak{k}) / I \rightarrow \operatorname{End}_{\mathbb{C}}(V)$ the associated *-representation of $U_{q}(\mathfrak{k}) / I$. Then

$$
\rho_{\sigma}=\bar{\rho} \circ \phi_{\sigma} \circ \pi_{\sigma}^{-1}: \mathcal{A}_{\sigma} \rightarrow \operatorname{End}\left(V_{\sigma}\right),
$$

with $V_{\sigma}=V$ as finite-dimensional Hilbert space, defines a *-representation of $\mathcal{A}_{\sigma}$ such that $\left.\rho_{\sigma}\right|_{\mathcal{E}}=\left.\rho\right|_{\mathcal{E}}$.

Remark 1.12. With the conventions of Corollary 1.11 we can define a $*$-representation $\rho_{\sigma, \tau}=$ $\bar{\rho} \circ \phi_{\tau} \circ \pi_{\sigma}^{-1}$ of $\mathcal{A}_{\sigma}$ for arbitrary $\sigma, \tau \in \mathbb{R}$. The $*$-representation $\rho_{\sigma, \tau}$ thus satisfies

$$
\rho_{\sigma, \tau}\left(B_{n}^{\sigma}\right)=\rho\left(\widehat{B}_{n}^{\tau}\right) .
$$

The special role of $\rho_{\sigma}=\rho_{\sigma, \sigma}$ follows from the fact that $B_{n}^{\sigma}$ and $\widehat{B}_{n}^{\sigma}$ are conjugate in $U_{q}(\mathfrak{g})$ (cf. Remark 1.7) and from the fact that the formal classical limit of $\rho_{\sigma}=\rho_{\sigma, \sigma}$ is equivalent to the formal classical limit of $\rho$ via the isomorphism $\widetilde{\mathcal{A}} \simeq U(\mathfrak{k})$ constructed in $\S 1.3$. 


\section{A. A. Oblomkov and J. V. Stokman}

Let $\kappa_{1} \in \mathbb{Z}$ and $\kappa \in \mathbb{Z}_{\geqslant 0}$. Denote by $V\left(\kappa, \kappa_{1}\right)$ the irreducible, finite-dimensional $U_{q}(\mathfrak{k})$-module

$$
V\left(\kappa, \kappa_{1}\right)=L_{\left(-\kappa_{1}\right)^{n}} \otimes L_{\left(\kappa_{1}+(n-1) \kappa,\left(\kappa_{1}-\kappa\right)^{n-1}\right)} .
$$

Here we have used the shorthand notation $\left(\kappa^{m}\right) \in P_{m}^{+}$for the $m$-tuple with all entries equal to $\kappa$. The representation map of $V\left(\kappa, \kappa_{1}\right)$ will be denoted by $\rho\left(\kappa, \kappa_{1}\right)$. Clearly there exists a scalar product on $V\left(\kappa, \kappa_{1}\right)$, unique up to constant multiples, such that $\rho\left(\kappa, \kappa_{1}\right): U_{q}(\mathfrak{k}) \rightarrow \operatorname{End}_{\mathbb{C}}\left(V\left(\kappa, \kappa_{1}\right)\right)$ is a $*$-representation. Since $L_{\left(-\kappa_{1}\right)^{n}}$ is one-dimensional, $x_{n-1} \in U_{q}(\mathfrak{g l}(n))$ acts as zero, and hence $I \subseteq \operatorname{Ker}\left(\rho\left(\kappa, \kappa_{1}\right)\right)$. Thus we obtain a $*$-representation

$$
\rho\left(\kappa, \kappa_{1}\right)_{\sigma}: \mathcal{A}_{\sigma} \rightarrow \operatorname{End}_{\mathbb{C}}\left(V\left(\kappa, \kappa_{1}\right)_{\sigma}\right)
$$

by the previous corollary. To simplify the notation, we denote

$$
\left(\rho\left(\kappa_{1}\right)_{\sigma}, V\left(\kappa_{1}\right)_{\sigma}\right)=\left(\rho\left(0, \kappa_{1}\right)_{\sigma}, V\left(0, \kappa_{1}\right)_{\sigma}\right) .
$$

The $U_{q}(\mathfrak{g l}(n))$-module $L_{\left((n-1) \kappa,(-\kappa)^{n-1}\right)}$ is the quantum analogue of the GL $(n ; \mathbb{C})$-module $S^{n \kappa}\left(\mathbb{C}^{n}\right)$ det $^{-\kappa}$, where $S^{m}\left(\mathbb{C}^{n}\right)$ is the space of homogeneous polynomials on $\mathbb{C}^{n}$ of degree $m$. This is precisely the $U_{q}(\mathfrak{g l}(n))$-module which plays the crucial role in Etingof's and Kirillov's [EK94] theory on $A$-type Macdonald polynomials and generalized quantum group characters. The module $L_{\left((n-1) \kappa,(-\kappa)^{n-1}\right)}$ admits a concrete description (see, e.g., [EK94, §5]) which can be directly lifted to $V\left(\kappa, \kappa_{1}\right)_{\sigma}$, leading to the following result.

Lemma 1.13. Let $\mathcal{J}_{\kappa}$ be the set of $n$-tuples $\underline{m}=\left(m_{1}, \ldots, m_{n}\right) \in \mathbb{Z}_{\geqslant 0}^{n}$ which sum up to $n \kappa$. There exists a $\sigma$-independent basis $\left\{r_{\underline{m}} \mid \underline{m} \in \mathcal{J}_{\kappa}\right\}$ of $V\left(\kappa, \kappa_{1}\right)$ such that

$$
\begin{aligned}
\rho\left(\kappa, \kappa_{1}\right)_{\sigma}\left(C_{i}\right) r_{\underline{m}} & =q^{m_{i}-\kappa} r_{\underline{m}}, \\
\rho\left(\kappa, \kappa_{1}\right)_{\sigma}\left(B_{j}\right) r_{\underline{m}} & =q^{-m_{j+1}+\kappa}\left(\frac{q^{m_{j}}-q^{-m_{j}}}{q-q^{-1}}\right) r_{\underline{m}-\varepsilon_{j}+\varepsilon_{j+1}}, \\
\rho\left(\kappa, \kappa_{1}\right)_{\sigma}\left(B_{2 n-j}\right) r_{\underline{m}} & =q^{-m_{j}+\kappa}\left(\frac{q^{m_{j+1}}-q^{-m_{j+1}}}{q-q^{-1}}\right) r_{\underline{m}+\varepsilon_{j}-\varepsilon_{j+1}}, \\
\rho\left(\kappa, \kappa_{1}\right)_{\sigma}\left(B_{n}^{\sigma}\right) r_{\underline{m}} & =\left(\frac{q^{-\sigma+2\left(\kappa-\kappa_{1}-m_{n}\right)}-q^{\sigma+2 \kappa_{1}}}{q-q^{-1}}\right) r_{\underline{m}}
\end{aligned}
$$

for $i=1, \ldots, n$ and $j=1, \ldots, n-1$, where we have used the convention that $r_{\underline{m}}=0$ if $\underline{m} \notin \mathcal{J}_{\kappa}$.

Lemma 1.13 implies that the common eigenspace

$$
\widetilde{V}\left(\kappa, \kappa_{1}\right)_{\sigma}=\left\{v \in V\left(\kappa, \kappa_{1}\right)_{\sigma} \mid \rho\left(\kappa, \kappa_{1}\right)_{\sigma}\left(C_{i}\right) v=v, \forall i\right\}
$$

is one-dimensional and spanned by $r_{\left(\kappa^{n}\right)}$.

The following lemma follows directly from Lemma 1.13.

Lemma 1.14. The $\mathcal{A}_{\sigma}$-module $V\left(\kappa, \kappa_{1}\right)_{\sigma}$, viewed as a $U_{q}(\mathfrak{g l}(n))$-module by restricting the module structure to $\mathcal{E} \subset \mathcal{A}_{\sigma}$ and using the isomorphism $\mathcal{E} \simeq U_{q}(\mathfrak{g l}(n))$ of Lemma 1.8, is isomorphic to the simple $U_{q}(\mathfrak{g l}(n))$-module $L_{\left((n-1) \kappa,(-\kappa)^{n-1}\right)}$. In particular, $V\left(\kappa, \kappa_{1}\right)_{\sigma}$ is a simple $\mathcal{A}_{\sigma}$-module.

We end this subsection by formulating branching rules for the quantum symmetric pair $\left(U_{q}(\mathfrak{g}), \mathcal{A}_{\sigma}\right)$. For the formulation it is convenient to define an injective map ${ }^{\natural}: \Lambda_{n} \rightarrow P_{2 n}$ by

$$
\mu^{\natural}=\left(\mu_{1}, \mu_{2}, \ldots, \mu_{n},-\mu_{n}, \ldots,-\mu_{2},-\mu_{1}\right) .
$$

Observe that ${ }^{\natural}$ restricts to a map ${ }^{\natural}: \Lambda_{n}^{+} \rightarrow P_{2 n}^{+}$, and that ${ }^{\natural}$ respects the dominance order, so $\mu^{\natural} \preceq \nu^{\natural}$ for $\mu, \nu \in \Lambda_{n}$ if $\mu \leqslant \nu$. 


\section{SPHERICAL FUnCTIONS AND MACDONALD-KOORNWINDER POLYNOMIALS}

Proposition 1.15. Let $\kappa_{2} \in \mathbb{Z}$ and $\kappa, \kappa_{1} \in \mathbb{Z}_{\geqslant 0}$ with $-\kappa_{1} \leqslant \kappa_{2} \leqslant \kappa_{1}$, and set

$$
\delta\left(\kappa, \kappa_{1}\right)=\left(\kappa_{1}+(n-1) \kappa, \kappa_{1}+(n-2) \kappa, \ldots, \kappa_{1}\right) \in \Lambda_{n}^{+} .
$$

Let $\lambda \in P_{2 n}^{+}$. The simple $\mathcal{A}_{\sigma}$-modules $V\left(\kappa, \kappa_{1}\right)_{\sigma}$ and $V\left(\kappa_{2}\right)_{\sigma}$ are both constituents of the $\mathcal{A}_{\sigma}$-module $L_{\lambda}^{\sigma}$ if and only if $\lambda \in\left(\Lambda_{n}^{+}+\delta\left(\kappa, \kappa_{1}\right)\right)^{\natural}$. For such $\lambda$, both $V\left(\kappa, \kappa_{1}\right)_{\sigma}$ and $V\left(\kappa_{2}\right)_{\sigma}$ occur with multiplicity one in $L_{\lambda}^{\sigma}$.

For $\kappa=\kappa_{1}=\kappa_{2}=0$, Proposition 1.15 states which of the simple, finite-dimensional $U_{q}(\mathfrak{g})$ modules $L_{\lambda}$ are spherical with respect to $\mathcal{A}_{\sigma}$. This was proven for general quantum symmetric pairs in [Let00, Theorem 4.3]. This special case can also be derived from the results in [NDS97], see Lemma 3.5. The classical case $(q=1)$ of Proposition 1.15 was proven in [Obl04] using the Littlewood-Richardson rule. Finally, for $n=1$ (in which case the parameter $\kappa$ is redundant), Proposition 1.15 is due to Koornwinder [Koo93], who used $q$-special functions for the proof. The general proof of Proposition 1.15 is discussed in $\S 2$.

Remark 1.16. In the remainder of this paper we always assume the conditions $\kappa, \kappa_{1} \in \mathbb{Z} \geqslant 0$ and $-\kappa_{1} \leqslant \kappa_{2} \leqslant \kappa_{1}$ on the representation labels $\kappa, \kappa_{1}, \kappa_{2} \in \mathbb{Z}$, and we use the shorthand notation $\vec{\kappa}=$ $\left(\kappa_{1}, \kappa_{2}, \kappa\right)$. There are three other parameter domains for $\vec{\kappa}$ for which similar results can be derived with only minor alterations, namely $\kappa_{1}, \kappa_{2} \in \mathbb{Z}, \kappa \in \mathbb{Z}_{\geqslant 0}$ satisfying $\kappa_{1} \in \mathbb{Z}_{\leqslant 0}$ and $\kappa_{1} \leqslant \kappa_{2} \leqslant-\kappa_{1}$, or $\kappa_{2} \in \mathbb{Z}_{\geqslant 0}$ and $-\kappa_{2} \leqslant \kappa_{1} \leqslant \kappa_{2}$, or $\kappa_{2} \in \mathbb{Z}_{\leqslant 0}$ and $\kappa_{2} \leqslant \kappa_{1} \leqslant-\kappa_{2}$ (cf. [Koe96] for the special case $n=1$ and [Obl04] for the classical case $(q=1))$.

\subsection{Vector valued spherical functions}

We write $G=\mathrm{GL}(2 n ; \mathbb{C})$ for the general linear group. The quantized algebra of regular functions $\mathbb{C}_{q}[G] \subseteq U_{q}(\mathfrak{g})^{*}$ is the span of the matrix coefficients of the irreducible $U_{q}(\mathfrak{g})$-representations $L_{\lambda}$ $\left(\lambda \in P_{2 n}^{+}\right)$. The quantized function algebra $\mathbb{C}_{q}[G]$ inherits from $U_{q}(\mathfrak{g})$ the structure of a Hopf *-algebra. In particular, the $*$-structure on $\mathbb{C}_{q}[G]$ is defined by

$$
f^{*}(X)=\overline{f\left(S(X)^{*}\right)}, \quad \forall X \in U_{q}(\mathfrak{g}) .
$$

The left and right regular $U_{q}(\mathfrak{g})$-action on $\mathbb{C}_{q}[G]$ is defined by

$$
(Y \cdot f \cdot Z)(X)=f(Z X Y), \quad X, Y, Z \in U_{q}(\mathfrak{g}) .
$$

The Peter-Weyl decomposition

$$
\mathbb{C}_{q}[G]=\bigoplus_{\lambda \in P_{2 n}^{+}} W(\lambda),
$$

with $W(\lambda)$ the span of the matrix coefficients of $L_{\lambda}$, is the irreducible decomposition of $\mathbb{C}_{q}[G]$ as $U_{q}(\mathfrak{g})$-bimodule. For any vector space $V$, we may and will view elements $f \in \mathbb{C}_{q}[G] \otimes V$ as linear maps $f: U_{q}(\mathfrak{g}) \rightarrow V$.

Recall the conventions and notations for the representation labels $\vec{\kappa}$ from Remark 1.16. We fix $\sigma, \tau \in \mathbb{R}$ once and for all, unless specified differently. In the following definition we identify $V\left(\kappa_{2}\right)_{\sigma} \simeq \mathbb{C}$ as vector spaces and view $\rho\left(\kappa_{2}\right)_{\sigma}$ as a character of $\mathcal{A}_{\sigma}$.

Definition 1.17. We call $f \in \mathbb{C}_{q}[G] \otimes V\left(\kappa, \kappa_{1}\right)_{\tau}$ a vector valued spherical function if, for all $X \in U_{q}(\mathfrak{g})$,

$$
\begin{aligned}
& f(X a)=\rho\left(\kappa_{2}\right)_{\sigma}(a) f(X), \quad \forall a \in \mathcal{A}_{\sigma}, \\
& f(b X)=\rho\left(\kappa, \kappa_{1}\right)_{\tau}(b) f(X), \quad \forall b \in \mathcal{A}_{\tau} .
\end{aligned}
$$

We denote by $F_{\vec{\kappa}}^{\sigma, \tau}$ the space of vector valued spherical functions.

By standard arguments (see, e.g., [Obl04] for the classical setup, and Lemma 4.1), we obtain the following corollary of Lemma 1.10 and Proposition 1.15. 


\section{A. A. Oblomkov and J. V. Stokman}

COROLlary 1.18. The space of vector valued spherical functions $F_{\vec{k}}^{\sigma, \tau}$ decomposes as

$$
F_{\vec{\kappa}}^{\sigma, \tau}=\bigoplus_{\mu \in \Lambda_{n}^{+}} F_{\vec{\kappa}}^{\sigma, \tau}(\mu)
$$

with $F_{\vec{\kappa}}^{\sigma, \tau}(\mu)$ the one-dimensional vector space

$$
F_{\vec{\kappa}}^{\sigma, \tau}(\mu)=F_{\vec{\kappa}}^{\sigma, \tau} \cap\left(W\left(\left(\mu+\delta\left(\kappa, \kappa_{1}\right)\right)^{\natural}\right) \otimes V\left(\kappa, \kappa_{1}\right)_{\tau}\right), \quad \mu \in \Lambda_{n}^{+} .
$$

We call a function $0 \neq f \in F_{\vec{\kappa}}^{\sigma, \tau}(\mu)$ an elementary vector valued spherical function of degree $\mu \in \Lambda_{n}^{+}$.

We consider now the restriction of vector valued spherical functions to the commutative subalgebra $U^{0} \subset U_{q}(\mathfrak{g})$ generated by the group-like elements $K_{j}^{ \pm 1}(j=1, \ldots, 2 n)$. Denote

$$
K^{\lambda}=K_{1}^{\lambda_{1}} K_{2}^{\lambda_{2}} \cdots K_{2 n}^{\lambda_{2 n}}, \quad \lambda \in P_{2 n},
$$

which form a linear basis of $U^{0}$, and define $\delta=\delta_{2 n} \in P_{2 n}^{+}$by

$$
\delta=(2 n-1,2 n-2, \ldots, 1,0) .
$$

Let $\mathbb{C}\left[z^{ \pm 1}\right]$ be the algebra of Laurent polynomials in $2 n$ variables $z_{1}, \ldots, z_{2 n}$.

Definition 1.19. Let $V$ be a complex vector space and $f \in \mathbb{C}_{q}[G] \otimes V$, viewed as the linear map $f: U_{q}(\mathfrak{g}) \rightarrow V$. We define a regular function $\left.f\right|_{T}:\left(\mathbb{C}^{*}\right)^{2 n} \rightarrow V$ (or, equivalently, $\left.f\right|_{T} \in \mathbb{C}\left[z^{ \pm 1}\right] \otimes V$ ) by the requirement that

$$
\left.f\right|_{T}\left(q^{\lambda}\right)=f\left(K^{\lambda-\delta}\right), \quad \forall \lambda \in P_{2 n},
$$

where $q^{\lambda}=\left(q^{\lambda_{1}}, \ldots, q^{\lambda_{2 n}}\right)$. The resulting linear map

$$
\left.\right|_{T}: \mathbb{C}_{q}[G] \otimes V \rightarrow \mathbb{C}\left[z^{ \pm 1}\right] \otimes V,\left.\quad f \mapsto f\right|_{T}
$$

is called the restriction map.

We define elements $u_{i} \in \mathbb{C}\left[z^{ \pm 1}\right]$ by

$$
u_{i}=z_{i} z_{2 n+1-i}^{-1}, \quad i=1, \ldots, n,
$$

and we write $\mathbb{C}\left[u^{ \pm 1}\right] \subset \mathbb{C}\left[z^{ \pm 1}\right]$ for the subalgebra generated by the $u_{i}^{ \pm 1}$. Recall the one-dimensional subspace $\widetilde{V}\left(\kappa, \kappa_{1}\right)_{\tau} \subseteq V\left(\kappa, \kappa_{1}\right)_{\tau}$ defined by $(1.15)$.

Lemma 1.20. If $f \in F_{\vec{\kappa}}^{\sigma, \tau} \subset \mathbb{C}_{q}[G] \otimes V\left(\kappa, \kappa_{1}\right)_{\tau}$, then $\left.f\right|_{T} \in \mathbb{C}\left[u^{ \pm 1}\right] \otimes \widetilde{V}\left(\kappa, \kappa_{1}\right)_{\tau}$. Identifying the one-dimensional vector space $\widetilde{V}\left(\kappa, \kappa_{1}\right)_{\tau}$ with $\mathbb{C}$, the restriction map thus gives rise to a linear map

$$
\left.\right|_{T}: F_{\vec{\kappa}}^{\sigma, \tau} \rightarrow \mathbb{C}\left[u^{ \pm 1}\right] .
$$

Proof. Fix $f \in F_{\vec{\kappa}}^{\sigma, \tau}$. For $i=1, \ldots, n$ and $X \in U^{0}$ we compute

$$
\begin{aligned}
\rho\left(\kappa, \kappa_{1}\right)_{\tau}\left(C_{i}\right) f(X) & =f\left(C_{i} X\right) \\
& =f\left(X C_{i}\right) \\
& =\rho\left(\kappa_{2}\right)_{\sigma}\left(C_{i}\right) f(X)=f(X),
\end{aligned}
$$

and hence $f(X) \in \widetilde{V}\left(\kappa, \kappa_{1}\right)_{\tau}$. Identifying $\widetilde{V}\left(\kappa, \kappa_{1}\right)_{\tau} \simeq \mathbb{C}$, we thus conclude that $\left.f\right|_{T} \in \mathbb{C}\left[z^{ \pm 1}\right]$. The formulas $f\left(X C_{i}\right)=f(X)$ for $X \in U^{0}$ and $i=1, \ldots, n$ imply that $\left.f\right|_{T} \in \mathbb{C}\left[u^{ \pm 1}\right]$.

The following main result of this paper gives a representation theoretic interpretation of the full five-parameter family of Macdonald-Koornwinder polynomials (with two parameters continuous, and three parameters discrete). 


\section{SPHERICAL FUnCTIONS AND MACDONALD-KOORNWINDER POLYNOMIALS}

Theorem 1.21. Fix parameters $\sigma, \tau \in \mathbb{R}$ and $\vec{\kappa}=\left(\kappa_{1}, \kappa_{2}, \kappa\right) \in \mathbb{Z}^{\times 3}$ with $\kappa, \kappa_{1} \in \mathbb{Z}_{\geqslant 0}$ and $-\kappa_{1} \leqslant$ $\kappa_{2} \leqslant \kappa_{1}$. Let $f_{0} \in F_{\vec{\kappa}}^{\sigma, \tau}(0)$ be an elementary vector valued spherical function of degree $0 \in \Lambda_{n}^{+}$.

(i) The restriction map $\left.\right|_{T}$ defines a linear bijection

$$
\left.\right|_{T}:\left.F_{\vec{\kappa}}^{\sigma, \tau} \rightarrow f_{0}\right|_{T} \mathbb{C}\left[u^{ \pm 1}\right]^{W} .
$$

(ii) We have

$$
\left.f_{0}\right|_{T}=C u^{\delta\left(\kappa, \kappa_{1}\right)} \prod_{i=1}^{n}\left(q^{1-\sigma+\tau} u_{i}^{-1} ; q^{2}\right)_{\kappa_{1}-\kappa_{2}}\left(-q^{1+\sigma+\tau} u_{i}^{-1} ; q^{2}\right)_{\kappa_{1}+\kappa_{2}} \prod_{1 \leqslant i<j \leqslant n}\left(q^{2} u_{i}^{-1} u_{j}, q^{2} u_{i}^{-1} u_{j}^{-1} ; q^{2}\right)_{\kappa}
$$

for some nonzero constant $C$.

(iii) If $f_{\mu} \in F_{\vec{\kappa}}^{\sigma, \tau}(\mu)$ is an elementary vector valued spherical function of degree $\mu \in \Lambda_{n}^{+}$, then

$$
\frac{\left.f_{\mu}\right|_{T}}{\left.f_{0}\right|_{T}}=D P_{\mu}\left(u ;-q^{\sigma+\tau+1+\kappa_{1}+\kappa_{2}},-q^{-\sigma-\tau+1}, q^{\sigma-\tau+1}, q^{-\sigma+\tau+1+\kappa_{1}-\kappa_{2}} ; q^{2}, q^{2 \kappa+2}\right)
$$

for some nonzero constant $D$.

Remark 1.22. In the special case $n=1$, Theorem 1.21 gives a representation theoretic interpretation of the Askey-Wilson polynomials, which is in accordance with the results from [Koo93], [NM90] and [Koe96]. The classical case $(q=1)$ of Theorem 1.21 was proven in [Obl04]. In $\S 3$ we show that Theorem 1.21 for $\kappa_{1}=\kappa_{2}=\kappa=0$ follows from the main results in [NDS97].

The proofs of Theorem 1.21(i), (ii) and (iii) are given in $\S 4, \S 5$ and $\S 6$, respectively.

\section{Branching rules}

The main goal of this section is to establish the specific branching rules for the quantum symmetric pairs $\left(U_{q}(\mathfrak{g}), \mathcal{A}_{\sigma}\right)$ as formulated in Proposition 1.15. We start by defining and studying the notion of the expectation value for the quantum symmetric pairs $\left(U_{q}(\mathfrak{g}), \mathcal{A}_{\sigma}\right)$.

\subsection{The expectation value}

The expectation value for intertwiners plays a crucial role in the representation theoretic approach to quantum field theory, see, e.g., [EV00]. The notion of expectation value is naturally associated to symmetric pairs $(H \times H, \operatorname{diag}(H))$ with $H$ a semisimple Lie group and $\operatorname{diag}(H)$ the diagonal embedding of $H$ in $H \times H$, as well as to their quantum analogues $\left(U_{q}(\mathfrak{h}) \otimes U_{q}(\mathfrak{h}), \Delta\left(U_{q}(\mathfrak{h})\right)\right)$ with $\mathfrak{h}$ a semisimple Lie algebra. In this subsection we define the expectation value for the quantum symmetric pair $\left(U_{q}(\mathfrak{g}), \mathcal{A}_{\sigma}\right)$. As we shall see, this directly leads to information on branching rules for the quantum symmetric pairs $\left(U_{q}(\mathfrak{g}), \mathcal{A}_{\sigma}\right)$.

For $\lambda \in P_{2 n}^{+}$we choose a highest weight vector $0 \neq v_{\lambda} \in L_{\lambda}[\lambda]$ and a lowest weight vector $0 \neq v_{w_{0} \lambda} \in L_{\lambda}\left[w_{0} \lambda\right]$, where $w_{0} \in S_{2 n}$ is the longest Weyl group element, $w_{0}(i)=2 n+1-i$ for all $i$. Both $v_{\lambda}$ and $v_{w_{0} \lambda}$ are unique up to a multiplicative constant.

Definition 2.1. Let $M$ be a left $\mathcal{A}_{\sigma}$-module and denote $\operatorname{Hom}_{\mathcal{A}_{\sigma}}\left(L_{\lambda}^{\sigma}, M\right)$ for the space of $\mathcal{A}_{\sigma}$-intertwiners $L_{\lambda}^{\sigma} \rightarrow M$. The maps $h_{M}^{\lambda}, l_{M}^{\lambda}: \operatorname{Hom}_{\mathcal{A}_{\sigma}}\left(L_{\lambda}^{\sigma}, M\right) \rightarrow M$, defined by $h_{M}^{\lambda}(\phi)=\phi\left(v_{\lambda}\right)$ and $l_{M}^{\lambda}(\phi)=\phi\left(v_{w_{0} \lambda}\right)$, are called the higher and lower expectation maps, respectively. The values $h_{M}^{\lambda}(\phi)$ and $l_{M}^{\lambda}(\phi)$ are called the higher and lower expectation values of $\phi$, respectively.

Define a map $b: P_{2 n} \rightarrow \Lambda_{n}$ by

$$
\lambda^{b}=\left(\lambda_{1}+\lambda_{2 n}, \lambda_{2}+\lambda_{2 n-1}, \ldots, \lambda_{n}+\lambda_{n+1}\right), \quad \lambda \in P_{2 n} .
$$




\section{A. A. Oblomkov and J. V. Stokman}

Observe that the kernel of b equals $\Lambda_{n}^{\natural}$ and that $\left(w_{0} \lambda\right)^{b}=\lambda^{b}$ for all $\lambda \in P_{2 n}$. If $M$ is a left $\mathcal{A}_{\sigma^{-} \text {-module }}$ and $\mu \in \Lambda_{n}$, then we define

$$
M_{\mu}=\left\{m \in M \mid C_{i} m=q^{\mu_{i}} m, \forall i=1, \ldots, n\right\} .
$$

Proposition 2.2. Let $M$ be a left $\mathcal{A}_{\sigma}$-module and let $\lambda \in P_{2 n}^{+}$. The expectation maps $h_{M}^{\lambda}$ and $l_{M}^{\lambda}$ are injective, with image contained in $M_{\lambda^{b}}$.

Proof. We prove the proposition for the expectation map $h_{M}^{\lambda}$, the proof for $l_{M}^{\lambda}$ is analogous. For $i=1, \ldots, n$ and $\phi \in \operatorname{Hom}_{\mathcal{A}_{\sigma}}\left(L_{\lambda}^{\sigma}, M\right)$ we have

$$
C_{i} h_{M}^{\lambda}(\phi)=C_{i} \phi\left(v_{\lambda}\right)=\phi\left(C_{i} v_{\lambda}\right)=q^{\lambda_{i}+\lambda_{2 n+1-i}} h_{M}^{\lambda}(\phi),
$$

so $h_{M}^{\lambda}(\phi) \in M_{\lambda^{b}}$. Thus the image of $h_{M}^{\lambda}$ is contained in $M_{\lambda^{b}}$.

Suppose $\phi \in \operatorname{Hom}\left(L_{\lambda}^{\sigma}, M\right)$ is in the kernel of $h_{M}^{\lambda}$, so $\phi\left(v_{\lambda}\right)=0$. We show that $\phi(v)=0$ for $v \in L_{\lambda}[\nu]$ and $\nu \preceq \lambda$ by induction on the height $\operatorname{ht}(\lambda-\nu) \in \mathbb{Z}_{\geqslant 0}$, where $\operatorname{ht}(\lambda-\nu)=\sum_{i=1}^{2 n-1} m_{i}$ if $m_{i}$ are the unique positive integers such that $\lambda-\nu=\sum_{i} m_{i} \alpha_{i}$. So suppose that $\phi(v)=0$ for all vectors $v \in L_{\lambda}[\nu]$ with $\nu \preceq \lambda$ and $\operatorname{ht}(\lambda-\nu) \leqslant N$.

For arbitrary $\nu \in P_{2 n}$ we have $L_{\lambda}[\nu]=\{0\}$ unless $\nu \preceq \lambda$ and

$$
L_{\lambda}[\nu]=\sum_{j=1}^{2 n-1} \widetilde{y}_{j} L_{\lambda}\left[\nu+\alpha_{j}\right],
$$

where $\widetilde{y}_{j}=y_{j} K_{j+1}^{-1} K_{2 n-j}^{-1}$. To prove the induction step, it thus suffices to show that $\phi\left(\widetilde{y}_{j} v\right)=0$ for all $j$ when $v \in L_{\lambda}[\nu], \nu \preceq \lambda$ and ht $(\lambda-\nu)=N$. If $v$ is such a vector, then by the explicit form of $B_{j}$ and $B_{n}^{\sigma}$ and by the induction hypothesis,

$$
\begin{aligned}
& \phi\left(\widetilde{y}_{j} v\right)=\phi\left(B_{j} v\right)=B_{j} \phi(v)=0, \\
& \phi\left(\widetilde{y}_{n} v\right)=\phi\left(B_{n}^{\sigma} v\right)=B_{n}^{\sigma} \phi(v)=0
\end{aligned}
$$

for $j \in\{1, \ldots, 2 n-1\} \backslash\{n\}$, as desired.

Since $L_{\lambda}^{\sigma}$ is $\mathcal{A}_{\sigma}$-semisimple by Lemma 1.10 , the previous proposition has the following immediate consequence.

Corollary 2.3. If $M$ is a simple $\mathcal{A}_{\sigma}$-module and $\lambda \in P_{2 n}^{+}$, then the number of summands isomorphic to $M$ in the irreducible decomposition of $L_{\lambda}^{\sigma}$ is bounded by $\operatorname{Dim}\left(M_{\lambda^{b}}\right)$.

Recall the conditions on the representation labels $\vec{\kappa}=\left(\kappa_{1}, \kappa_{2}, \kappa\right)$ from Remark 1.16.

Lemma 2.4. Let $\lambda \in P_{2 n}^{+}$. If both $V\left(\kappa, \kappa_{1}\right)_{\sigma}$ and $V\left(\kappa_{2}\right)_{\sigma}$ occur as constituents in the irreducible decomposition of the $\mathcal{A}_{\sigma}$-semisimple module $L_{\lambda}^{\sigma}$, then $\lambda \in\left(\Lambda_{n}^{+}+\delta\left(\kappa, \kappa_{1}\right)\right)^{\natural}$. For such $\lambda$, the modules $V\left(\kappa, \kappa_{1}\right)_{\sigma}$ and $V\left(\kappa_{2}\right)_{\sigma}$ occur at most with multiplicity one in $L_{\lambda}^{\sigma}$.

Proof. The weight spaces $M_{\mu}$ (see (2.1)) for the simple $\mathcal{A}_{\sigma}$-modules $M=V\left(\kappa, \kappa_{1}\right)_{\sigma}$ and $M=V\left(\kappa_{2}\right)_{\sigma}$ are at most one-dimensional by Lemma 1.13. Thus, for any $\lambda \in P_{2 n}^{+}$, the number of summands isomorphic to $V\left(\kappa, \kappa_{1}\right)_{\sigma}$ or to $V\left(\kappa_{2}\right)_{\sigma}$ in the irreducible decomposition of $L_{\lambda}^{\sigma}$ is at most one by Corollary 2.3.

If $v \in V\left(\kappa_{2}\right)_{\sigma}$ then $C_{i} v=v$ for all $i$; hence, $V\left(\kappa_{2}\right)_{\sigma}$ can only occur as a constituent in the irreducible decomposition of $L_{\lambda}^{\sigma}$ when $\lambda \in P_{2 n}^{+} \cap \operatorname{Ker}(b)=\left(\Lambda_{n}^{+}\right)^{\natural}$.

Fix $\mu \in \Lambda_{n}^{+}$such that $V\left(\kappa, \kappa_{1}\right)_{\sigma}$ occurs as a constituent of the irreducible decomposition of $L_{\mu^{\natural}}^{\sigma}$. It remains to show that this assumption imposes the additional restrictions $\mu_{n} \geqslant \kappa_{1}$ and $\mu_{j}-\mu_{j+1} \geqslant \kappa(j=1, \ldots, n-1)$ on $\mu$. Throughout the proof, we fix a nonzero intertwiner $\phi \in \operatorname{Hom}_{\mathcal{A}_{\sigma}}\left(L_{\mu^{\natural}}^{\sigma}, V\left(\kappa, \kappa_{1}\right)_{\sigma}\right)$. 


\section{SPHERICAL FUnCTIONS AND MACDONALD-KOORNWINDER POLYNOMIALS}

We start with proving $\mu_{n} \geqslant \kappa_{1}$. We use the notation of Remark 1.7. Standard $U_{q}(\mathfrak{g l}(2))$ representation theory implies that $V=U_{q}(\mathfrak{g l}(2))^{(n)} v_{\mu^{\natural}} \subseteq L_{\mu^{\natural}}$ is the $\left(2 \mu_{n}+1\right)$-dimensional irreducible representation of $U_{q}(\mathfrak{g l}(2))^{(n)} \simeq U_{q}(\mathfrak{g l}(2))$ with highest weight $\left(\mu_{n},-\mu_{n}\right)$ and highest weight vector $v_{\mu^{\natural}}$, and that $\left.\widehat{B}_{n}^{\sigma}\right|_{V} \in \operatorname{End}_{\mathbb{C}}(V)$ is semisimple with simple spectrum

$$
s_{l}=\frac{q^{-\sigma+2 l}-q^{\sigma-2 l}}{q-q^{-1}}, \quad l=-\mu_{n}, \ldots, \mu_{n}-1, \mu_{n} .
$$

Observe, furthermore, that $V$ is $x_{\sigma}$-stable. Since $x_{\sigma} B_{n}^{\sigma} x_{\sigma}^{-1}=\widehat{B}_{n}^{\sigma}$, it follows that $\left.B_{n}^{\sigma}\right|_{V} \in \operatorname{End}_{\mathbb{C}}(V)$ is semisimple with simple spectrum $\left\{s_{l} \mid l=-\mu_{n}, \ldots, \mu_{n}-1, \mu_{n}\right\}$. Proposition 2.2 and the fact that $C_{i}$ centralizes $U_{q}(\mathfrak{g l}(2))^{(n)}$ for $i=1, \ldots, n$ imply that $\phi(V)=\widetilde{V}\left(\kappa, \kappa_{1}\right)_{\sigma}$ with $\widetilde{V}\left(\kappa, \kappa_{1}\right)_{\sigma}$ the one-dimensional space defined by (1.15). On the other hand, Lemma 1.13 shows that $\widetilde{V}\left(\kappa, \kappa_{1}\right)_{\sigma}$ is the eigenspace of $\rho\left(\kappa, \kappa_{1}\right)_{\sigma}\left(B_{n}^{\sigma}\right)$ with eigenvalue $s_{-\kappa_{1}}$; hence $s_{-\kappa_{1}}$ must be in the spectrum of $\left.B_{n}^{\sigma}\right|_{V}$. Consequently, $\mu_{n} \geqslant \kappa_{1}$.

Next we show that $\mu_{j}-\mu_{j+1} \geqslant \kappa$ for $j=1, \ldots, n-1$. Fix $j \in\{1, \ldots, n-1\}$. Since $0 \neq$ $\phi\left(v_{\mu^{\natural}}\right) \in \widetilde{V}\left(\kappa, \kappa_{1}\right)_{\sigma}$ by Proposition 2.2, we conclude from the explicit description of $V\left(\kappa, \kappa_{1}\right)_{\sigma}$ (see Lemma 1.13) that

$$
\phi\left(B_{j}^{\kappa} v_{\mu^{\natural}}\right)=\rho\left(\kappa, \kappa_{1}\right)_{\sigma}\left(B_{j}^{\kappa}\right) \phi\left(v_{\mu^{\natural}}\right) \neq 0 .
$$

This implies that $B_{j}^{\kappa} v_{\mu^{\natural}} \neq 0$ in $L_{\mu^{\natural}}$. We write $B_{j}=\widetilde{y}_{j}+\widetilde{x}_{2 n-j}$ with

$$
\widetilde{y}_{j}=y_{j} K_{j+1}^{-1} K_{2 n-j}^{-1}, \quad \widetilde{x}_{2 n-j}=K_{j}^{-1} x_{2 n-j} K_{2 n-j}^{-1} .
$$

Since $v_{\mu^{\natural}} \in L_{\mu^{\natural}}$ is a highest weight vector, the commutation relation $\widetilde{x}_{2 n-j} \widetilde{y}_{j}=q^{2} \widetilde{y}_{j} \widetilde{x}_{2 n-j}$ in $U_{q}(\mathfrak{g})$ implies

$$
\widetilde{y}_{j}^{\kappa} v_{\mu^{\natural}}=B_{j}^{\kappa} v_{\mu^{\natural}} \neq 0,
$$

and consequently $y_{j}^{\kappa} v_{\mu^{\natural}} \neq 0$. Standard arguments from $U_{q}(\mathfrak{g l}(2)) \simeq \mathbb{C}\left\langle x_{j}, y_{j}, K_{j}^{ \pm 1}, K_{j+1}^{ \pm 1}\right\rangle$ representation theory imply that $\mu_{j}-\mu_{j+1}$ is the largest positive integer $m$ for which $y_{j}^{m} v_{\mu^{\natural}} \neq 0$ in $L_{\mu^{\natural}}$. We conclude that $\mu_{j}-\mu_{j+1} \geqslant \kappa$, as desired.

In, for example, [Nou96] and [NDS97], highest weight considerations are used to determine branching rules for quantum symmetric pairs. These highest weight arguments can be formalized and generalized in the present setting as follows.

Lemma 2.5. Let $\lambda \in P_{2 n}^{+}$and let $\{0\} \neq M \subseteq L_{\lambda}^{\sigma}$ be an $\mathcal{A}_{\sigma}$-submodule. Then

$$
M \nsubseteq \bigoplus_{\nu \prec \lambda} L_{\lambda}[\nu], \quad M \nsubseteq \bigoplus_{\nu \succ w_{0} \lambda} L_{\lambda}[\nu] .
$$

In other words, there exists a vector $m_{+} \in M$ (respectively $m_{-} \in M$ ) whose decomposition in $U^{0}$-weights has a nonzero highest (respectively lowest) weight contribution.

Proof. Suppose $M \subseteq L_{\lambda}^{\sigma}$ is an $\mathcal{A}_{\sigma}$-submodule and

$$
M \subseteq \bigoplus_{\nu \prec \lambda} L_{\lambda}[\nu]
$$

Let $M^{\perp}$ be the orthocomplement of $M$ in $L_{\lambda}$ with respect to the $*$-unitary scalar product $\langle\cdot, \cdot\rangle_{\lambda}$ on $L_{\lambda}$. Then $M^{\perp}$ is an $\mathcal{A}_{\sigma}$-module complement of $M$ in $L_{\lambda}^{\sigma}$. Let $\pi \in \operatorname{Hom}_{\mathcal{A}_{\sigma}}\left(L_{\lambda}^{\sigma}, M\right)$ be the projection onto $M$ along $M^{\perp}$. By the $*$-self-adjointness of the $K_{i}$, we have $L_{\lambda}[\nu] \perp L_{\lambda}\left[\nu^{\prime}\right]$ for $\nu \neq \nu^{\prime}$. Condition (2.2) thus implies $L_{\lambda}[\lambda] \subseteq M^{\perp}$. Hence the higher expectation value $h_{M}^{\lambda}(\pi)$ is zero. Proposition 2.2 then implies $\pi \equiv 0$, hence $M=\{0\}$. The second statement is proved similarly. 


\section{A. A. Oblomkov and J. V. Stokman}

Remark 2.6. For an arbitrary quantum symmetric pair $\left(U, B_{\theta}\right)$ in the sense of Letzter (see [Let03, $\S 7]$ for a complete list), one can make a similar definition of higher and lower expectation maps and of expectation values. Following the reasoning of this section, one can establish the analogues of Proposition 2.2, Corollary 2.3 and Lemma 2.5 in this general setup (cf. the proof of [Let04, Lemma 5.6]).

\subsection{Deformation arguments}

In this subsection we complete the proof of Proposition 1.15 by using deformation arguments. Without loss of generality we may take $q=e^{h}$ to be a formal deformation parameter. We use part four of Kassel's book [Kas95] as main reference for facts and the notation related to topological $\mathbb{C}[[h]]$-modules and topological $\mathbb{C}[[h]]$-algebras.

Let $U_{h}(\mathfrak{g})$ be the topological version of the quantized universal enveloping algebra $U_{q}(\mathfrak{g})$, with topological generators $E_{i, i}, x_{j}$ and $y_{j}(i \in\{1, \ldots, 2 n\}, j \in\{1, \ldots, 2 n-1\})$, where $K_{i}=e^{h E_{i, i}}$. Let $U_{h}(\mathfrak{s l}(2 n))$ be the subalgebra of $U_{h}(\mathfrak{g l}(2 n))$ topologically generated by the elements $E_{j, j}-E_{j+1, j+1}$, $x_{j}$ and $y_{j}(j \in\{1, \ldots, 2 n-1\})$. Note that $U_{h}(\mathfrak{g})$ is the central extension of $U_{h}(\mathfrak{s l}(2 n))$ by the central element $E_{1,1}+E_{2,2}+\cdots+E_{2 n, 2 n}$.

The topological version of $\mathcal{A}_{\sigma}$ is the subalgebra of $U_{h}(\mathfrak{g})$ topologically generated by $B_{j}, B_{n}^{\sigma}$ and $E_{i, i}+E_{2 n+1-i, 2 n+1-i}$ for $j \in\{1, \ldots, 2 n-1\} \backslash\{n\}$ and $i \in\{1, \ldots, n\}$. We denote by $\overline{\mathcal{A}}_{\sigma}$ the subalgebra of $U_{h}(\mathfrak{s l}(2 n))$ topologically generated by $B_{j} C_{j+1}, B_{n}^{\sigma} C_{n}$ and $E_{i, i}-E_{i+1, i+1}-E_{2 n-i, 2 n-i}+$ $E_{2 n+1-i, 2 n+1-i}$ for $j \in\{1, \ldots, 2 n-1\} \backslash\{n\}$ and $i \in\{1, \ldots, n-1\}$. Note that $\mathcal{A}_{\sigma}$ is the central extension of $\overline{\mathcal{A}}_{\sigma}$ by the central element $E_{1,1}+E_{2,2}+\cdots+E_{2 n, 2 n} \in U_{h}(\mathfrak{g})$.

We fix $\lambda=\left(\mu+\delta\left(\kappa, \kappa_{1}\right)\right)^{\natural}$ with $\mu \in \Lambda_{n}^{+}$. To complete the proof of Proposition 1.15, it suffices to show that the irreducible $\mathcal{A}_{\sigma}$-module $V\left(\kappa, \kappa_{1}\right)_{\sigma}$ occurs as a constituent of $L_{\lambda}^{\sigma}$. Since the central element $E_{1,1}+\cdots+E_{2 n, 2 n}$ acts as zero on the topological versions of $V\left(\kappa, \kappa_{1}\right)_{\sigma}$ and $L_{\lambda}$, it suffices to show that $V\left(\kappa, \kappa_{1}\right)_{\sigma}$ is a constituent of $L_{\lambda}^{\sigma}$ when viewed as $\overline{\mathcal{A}}_{\sigma}$-modules.

The formal computations after Definition 1.5 and [Let02, Theorem 7.5] imply that the algebra $\overline{\mathcal{A}}_{\sigma}$ is a deformation of the universal enveloping algebra $U(\overline{\mathfrak{k}})$ with

$$
\overline{\mathfrak{k}}=\operatorname{Ad}(g)(\mathfrak{k}) \cap \mathfrak{s l}(2 n)=\overline{\mathfrak{k}}_{s s} \oplus \mathbb{C} Z,
$$

where $g$ is given by $(1.11), \overline{\mathfrak{k}}_{s s}=\operatorname{Ad}(g)(\mathfrak{s l}(n) \oplus \mathfrak{s l}(n))$, and $Z \in \overline{\mathfrak{k}}$ is the central element

$$
Z=\operatorname{Ad}(g)\left(Z^{\prime}\right)=-\frac{1}{2 n} \sum_{j=1}^{2 n} E_{j, 2 n+1-j}
$$

where

$$
Z^{\prime}=-\frac{1}{2 n}\left(E_{1,1}+E_{2,2}+\cdots+E_{n, n}-E_{n+1, n+1}-E_{n+2, n+2}-\cdots-E_{2 n, 2 n}\right) .
$$

Since $\overline{\mathfrak{k}}$ is reductive with one-dimensional centre, we have $H^{2}(\overline{\mathfrak{k}}, U(\overline{\mathfrak{k}}))=\{0\}$. By [Kas95, Theorem XVIII.2.2] there exists a topological algebra isomorphism

$$
\beta_{\sigma}: \overline{\mathcal{A}}_{\sigma} \rightarrow U(\overline{\mathfrak{k}})[[h]]
$$

which is the identity modulo $h$. We define

$$
\overline{\mathcal{A}}_{\sigma}^{s s}=\beta_{\sigma}^{-1}\left(U\left(\overline{\mathfrak{k}}_{s s}\right)[[h]]\right), \quad Z_{\sigma}=\beta_{\sigma}^{-1}(Z),
$$

so then $\overline{\mathcal{A}}_{\sigma}^{s s}$ is a deformation of $U\left(\overline{\mathfrak{k}}_{s s}\right), Z_{\sigma}$ is central in $\overline{\mathcal{A}}_{\sigma}$ and $\overline{\mathcal{A}}_{\sigma}$ is topologically generated by $\overline{\mathcal{A}}_{\sigma}^{s s}$ and $Z_{\sigma}$. We denote by $\left(\beta_{\sigma}^{-1}\right)^{*}\left(V\left(\kappa, \kappa_{1}\right)_{\sigma}\right)$ the module $V\left(\kappa, \kappa_{1}\right)_{\sigma}$ viewed as a module over $U(\overline{\mathfrak{k}})[[h]]$ via the isomorphism $\beta_{\sigma}: \overline{\mathcal{A}}_{\sigma} \rightarrow U(\overline{\mathfrak{k}})[[h]]$ (we will use a similar notation to indicate the pull back of an action by some algebra morphism). The way we constructed the module $V\left(\kappa, \kappa_{1}\right)_{\sigma}$ in $\S 1$ immediately implies that, modulo $h$, we re-obtain the irreducible, finite-dimensional $(\mathfrak{g l}(n) \times \mathfrak{g l}(n)) \cap \mathfrak{s l}(2 n) \simeq \overline{\mathfrak{k}}$ 


\section{SPHERICAL FUnCTIONS AND MACDONALD-KOORNWINDER POLYNOMIALS}

module $V^{\text {clas }}\left(\kappa, \kappa_{1}\right)$ of highest weight

$$
\left(\left(-\kappa_{1}\right)^{n},\left(\kappa_{1}+(n-1) \kappa,\left(\kappa_{1}-\kappa\right)^{n-1}\right)\right) \in P_{n}^{+} \times P_{n}^{+} .
$$

Observe that the central element $Z$ acts as multiplication by $\kappa_{1}$ on $V^{\text {clas }}\left(\kappa, \kappa_{1}\right)$, while $V^{\text {clas }}\left(\kappa, \kappa_{1}\right)$ is independent of $\kappa_{1}$ when viewed as module over $\overline{\mathfrak{E}}_{s s}$. Since $\overline{\mathfrak{E}}_{s s}$ is semisimple, we conclude that $\left(\beta_{\sigma}^{-1}\right)^{*}\left(V\left(\kappa, \kappa_{1}\right)_{\sigma}\right)$, viewed as the $U\left(\overline{\mathfrak{E}}_{s s}\right)[[h]]$-module, is isomorphic to the $\mathbb{C}[[h]]$-linear extension of the $\overline{\mathfrak{k}}_{s s}$-module $V^{\text {clas }}\left(\kappa, \kappa_{1}\right)$.

Lemma 2.7. There exists a $\overline{\mathcal{A}}_{\sigma}$-submodule $\bar{V}\left(\kappa, \kappa_{1}\right)_{\sigma} \subset L_{\lambda}^{\sigma}$ such that:

(i) $\bar{V}\left(\kappa, \kappa_{1}\right)_{\sigma} \simeq V\left(\kappa, \kappa_{1}\right)_{\sigma}$ as $\overline{\mathcal{A}}_{\sigma}^{s s}$-modules;

(ii) the central element $Z_{\sigma}$ acts as multiplication by a scalar $\bar{\xi}_{\sigma} \in \mathbb{C}[[h]]$ on $\bar{V}\left(\kappa, \kappa_{1}\right)_{\sigma}$;

(iii) modulo $h, \bar{V}\left(\kappa, \kappa_{1}\right)_{\sigma}$ is the $\overline{\mathfrak{k}}$-module $V^{\text {clas }}\left(\kappa, \kappa_{1}\right)$.

Proof. Since $U_{h}(\mathfrak{s l}(2 n))$ is a deformation of the universal enveloping algebra of the simple Lie algebra $\mathfrak{s l}(2 n)$, there exists by [Kas95, Theorem XVIII.2.2] a topological algebra isomorphism

$$
\alpha: U_{h}(\mathfrak{s l}(2 n)) \rightarrow U(\mathfrak{s l}(2 n))[[h]]
$$

which is the identity modulo $h$. The natural embedding $\iota_{\sigma}: \overline{\mathcal{A}}_{\sigma} \rightarrow U_{h}(\mathfrak{s l}(2 n))$ of topological algebras can thus be pulled back to obtain an embedding

$$
\gamma_{\sigma}=\alpha \circ \iota_{\sigma} \circ \beta_{\sigma}^{-1}: U(\overline{\mathfrak{k}})[[h]] \rightarrow U(\mathfrak{s l}(2 n))[[h]]
$$

of topological algebras, which is the identity modulo $h$. Since $\overline{\mathfrak{k}}_{s s}$ is semisimple, we have $H^{1}\left(\overline{\mathfrak{k}}_{s s}\right.$, $U(\mathfrak{s l}(2 n)))=\{0\}$, and hence there exists an invertible element $F_{\sigma} \in U(\mathfrak{s l}(2 n))[[h]]$ which is 1 modulo $h$ such that

$$
\gamma_{\sigma}(X)=F_{\sigma} X F_{\sigma}^{-1}, \quad \forall X \in U\left(\overline{\mathfrak{k}}_{s s}\right)[[h]] \subset U(\overline{\mathfrak{k}})[[h]] \subset U(\mathfrak{s l}(2 n))[[h]]
$$

by [Kas95, Theorem XVIII.2.1].

Let $L_{\lambda}^{\text {clas }}$ be the irreducible $U(\mathfrak{s l}(2 n))$-module corresponding to the highest weight $\lambda$. We denote $V \subset L_{\lambda}^{\text {clas }}$ for its $V^{\text {clas }}\left(\kappa, \kappa_{1}\right)$-isotypical component viewed as a $U\left(\overline{\mathfrak{k}}_{s s}\right)$-module. The central element $Z \in \overline{\mathfrak{k}}$ acts semisimply on $V$ and the corresponding $Z$-eigenspaces $V_{r}$ of $V$ (with $r$ the $Z$-eigenvalue) yield the $V^{\text {clas }}(\kappa, r)$-isotypical components of $L_{\lambda}^{\text {clas }}$ viewed now as a $\overline{\mathfrak{k}}$-module. By the classical branching rules [Obl04, Lemma 3] for the symmetric pair $(\mathfrak{g}, \mathfrak{g l}(n) \times \mathfrak{g l}(n))$ we have $V_{\kappa_{1}} \simeq V^{\text {clas }}\left(\kappa, \kappa_{1}\right)$.

Let $L_{\lambda}^{\text {clas }}[[h]]$ be the $U(\mathfrak{s l}(2 n))[[h]]$-module obtained by $\mathbb{C}[[h]]$-linear extension of the $U(\mathfrak{s l}(2 n))$ module structure on $L_{\lambda}^{\text {clas }}$. Then $L_{\lambda}^{\sigma}$ is isomorphic to $\left(\alpha \circ \iota_{\sigma}\right)^{*}\left(L_{\lambda}^{\text {clas }}[[h]]\right)$ as topological $\overline{\mathcal{A}}_{\sigma}$-module. Now consider the $U(\overline{\mathfrak{E}})[[h]]$-module

$$
\left(\beta_{\sigma}^{-1}\right)^{*}\left(L_{\lambda}^{\sigma}\right)=\gamma_{\sigma}^{*}\left(L_{\lambda}^{\text {clas }}[[h]]\right)
$$

By $(2.4), F_{\sigma} V[[h]]$ is the $V^{\text {clas }}\left(\kappa, \kappa_{1}\right)[[h]]$-isotypical component of the $U\left(\overline{\mathfrak{k}}_{s s}\right)[[h]]$-module $\gamma_{\sigma}^{*}\left(L_{\lambda}^{\text {clas }}[[h]]\right)$. The proof is now completed by observing that $F_{\sigma} V[[h]]$ contains a nonzero $Z$-eigenspace $\bar{V}\left(\kappa, \kappa_{1}\right)_{\sigma}$ for some eigenvalue $\bar{\xi}_{\sigma} \in \mathbb{C}[[h]]$ satisfying $\bar{\xi}_{\sigma}=\kappa_{1}$ modulo $h$.

The central element $Z_{\sigma} \in \overline{\mathcal{A}}_{\sigma}$ acts by a scalar $\xi_{\sigma} \in \mathbb{C}[[h]]$ on $V\left(\kappa, \kappa_{1}\right)_{\sigma}$. To complete the arguments it thus remains to prove the following lemma.

Lemma 2.8. $\xi_{\sigma}=\bar{\xi}_{\sigma}$ in $\mathbb{C}[[h]]$.

Proof. We use the fact that $B_{n}^{\sigma} C_{n} \in \overline{\mathcal{A}}_{\sigma}$ is conjugate to the Cartan type element

$$
\bar{B}_{n}^{\sigma}=\frac{q^{-\sigma} K_{n} K_{n+1}^{-1}-q^{\sigma} K_{n}^{-1} K_{n+1}}{q-q^{-1}}
$$




\section{A. A. Oblomkov and J. V. Stokman}

in $U_{h}(\mathfrak{s l}(2 n))$, see [Ros00] and Remark 1.7. We first show that $B_{n}^{\sigma} C_{n}$ acts semisimply on $\bar{V}\left(\kappa, \kappa_{1}\right)_{\sigma}$, with spectrum contained in

$$
\left\{t_{l}=\frac{q^{-\sigma+l}-q^{\sigma-l}}{q-q^{-1}} \mid l \in \mathbb{Z}\right\} .
$$

Indeed, $B_{n}^{\sigma} C_{n}$ acts semisimply on $L_{\lambda}^{\sigma}$, with spectrum contained in $\left\{t_{l}\right\}_{l \in \mathbb{Z}}$. Hence any vectors $v \in$ $\bar{V}\left(\kappa, \kappa_{1}\right)_{\sigma}$ can be written as $v=\sum_{l} v_{l}$ with $v_{l} \in \operatorname{Ker}\left(B_{n}^{\sigma} C_{n}-t_{l}\right) \subseteq L_{\lambda}^{\sigma}$. Since $B_{n}^{\sigma} C_{n}$ preserves $\bar{V}\left(\kappa, \kappa_{1}\right)_{\sigma}$, we have $\left(B_{n}^{\sigma} C_{n}\right)^{k}(v)=\sum_{l} t_{l}^{k} v_{l} \in \bar{V}\left(\kappa, \kappa_{1}\right)_{\sigma}$ for all $k \in \mathbb{Z}_{\geqslant 0}$. Simple calculations involving the Vandermonde determinant show that there exist $c_{k}^{l} \in \mathbb{C}[[h]]$ such that $v_{l}=\sum_{k} c_{k}^{l}\left(B_{n}^{\sigma} C_{n}\right)^{k} v$ for all $l$, and hence $v_{l} \in \bar{V}\left(\kappa, \kappa_{1}\right)_{\sigma}$ for all $l$. By Lemma 1.13, the same statement about the spectrum is valid for the action of $B_{n}^{\sigma} C_{n}$ on $V\left(\kappa, \kappa_{1}\right)_{\sigma}$.

Lemma 2.7 and the fact that the eigenvalues $\left.t_{l}\right|_{h=0}=\sigma-l(l \in \mathbb{Z})$ remain separated modulo $h$ imply that the dimensions over $\mathbb{C}[[h]]$ of the $B_{n}^{\sigma} C_{n}$-eigenspaces of $V\left(\kappa, \kappa_{1}\right)_{\sigma}$ and $\bar{V}\left(\kappa, \kappa_{1}\right)_{\sigma}$ corresponding to a given eigenvalue $t_{l}$ are the same. In particular, we have

$$
\operatorname{Tr}_{V\left(\kappa, \kappa_{1}\right)_{\sigma}}\left(B_{n}^{\sigma} C_{n}\right)=\operatorname{Tr}_{\bar{V}\left(\kappa, \kappa_{1}\right)_{\sigma}}\left(B_{n}^{\sigma} C_{n}\right) .
$$

Next we expand $B_{n}^{\sigma} C_{n}$ in its semisimple and central parts

$$
B_{n}^{\sigma} C_{n}=\sum_{j=0}^{\infty} a_{j} Z_{\sigma}^{j}
$$

in $\overline{\mathcal{A}}_{\sigma}$, with $a_{j} \in \overline{\mathcal{A}}_{\sigma}^{s s}$. Then (2.5) leads to the equality

$$
\sum_{j=0}^{\infty} b_{j} \xi_{\sigma}^{j}=\sum_{j=0}^{\infty} b_{j} \bar{\xi}_{\sigma}^{j}
$$

as a formal power series in $h$, with

$$
b_{j}=\operatorname{Tr}_{V\left(\kappa, \kappa_{1}\right)_{\sigma}}\left(a_{j}\right) \in \mathbb{C}[[h]] .
$$

Now the computations after Definition 1.5 show that

$$
\operatorname{Ad}(g)\left(\left.B_{n}^{\sigma} C_{n}\right|_{h=0}\right)=E_{n, n}-E_{n+1, n+1}-\sigma 1=\left.a_{0}\right|_{h=0}+\left.a_{1}\right|_{h=0} Z^{\prime}
$$

with

$$
\begin{aligned}
\left.a_{0}\right|_{h=0}= & -\frac{1}{n} E_{1,1}-\cdots-\frac{1}{n} E_{n-1, n-1}+\frac{n-1}{n} E_{n, n} \\
& +\frac{1-n}{n} E_{n+1, n+1}+\frac{1}{n} E_{n+2, n+2}+\cdots+\frac{1}{n} E_{2 n, 2 n}-\sigma 1
\end{aligned}
$$

and $\left.a_{1}\right|_{h=0}=-2$. Thus $\left.b_{1}\right|_{h=0} \neq 0$ and $\left.b_{j}\right|_{h=0}=0$ for $j \geqslant 2$. Now we can expand (2.6) in powers of $h$, and compare coefficients of $h^{k}$ on each side. An easy induction argument using $\left.b_{1}\right|_{h=0} \neq 0$ and $\left.b_{j}\right|_{h=0}=0$ for $j \geqslant 2$ then leads to $\xi_{\sigma}=\bar{\xi}_{\sigma}$ (this may be viewed as a formal version of the inverse function theorem).

\section{Zonal spherical functions}

The purpose of this section is to establish Theorem 1.21 for $\vec{\kappa}=\overrightarrow{0}=(0,0,0)$. This is done by translating the results for zonal spherical functions on quantum Grassmannians proved in [NDS97] and [DS99] to the present setting. In [NDS97] and [DS99], extensive use is made of the $L$-operators of $U_{q}(\mathfrak{g})$. Roughly speaking, the $L$-operators $L^{ \pm}$for $U_{q}(\mathfrak{g})$ are the $2 n \times 2 n$ matrices with entries in $U_{q}(\mathfrak{g})$ obtained from the universal $R$-matrix of $U_{q}(\mathfrak{g})$ by applying the vector representation of $U_{q}(\mathfrak{g})$ to one of its components. We refer to Letzter [Let99, § 6] and Noumi [Nou96] for precise definitions; here we only recall those properties of the $L$-operators which are used in this paper. 


\section{SPHERICAL FUnCTIONS AND MACDONALD-KOORNWINDER POLYNOMIALS}

The matrix $L^{+}$(respectively $L^{-}$) is upper (respectively lower) triangular. If $l_{i j}^{ \pm} \in U_{q}(\mathfrak{g})$ is the $(i, j)$ th matrix coefficient of $L^{ \pm}$, then

$$
\begin{gathered}
l_{i i}^{+}=K_{i}, \quad l_{i i}^{-}=K_{i}^{-1}, \\
l_{j, j+1}^{+}=\left(q-q^{-1}\right) K_{j} y_{j}, \quad l_{j+1, j}^{-}=\left(q^{-1}-q\right) x_{j} K_{j}^{-1}
\end{gathered}
$$

for $i=1, \ldots, 2 n$ and $j=1, \ldots, 2 n-1$. More generally, $l_{i j}^{+}$(respectively $l_{i j}^{-}$) is an analogue of the root vector of $\mathfrak{g}$ corresponding to the root $\epsilon_{i}-\epsilon_{j}$ (respectively $\epsilon_{j}-\epsilon_{i}$ ). Furthermore,

$$
\Delta\left(l_{i j}^{ \pm}\right)=\sum_{k=1}^{2 n} l_{i k}^{ \pm} \otimes l_{k j}^{ \pm}, \quad \epsilon\left(l_{i j}^{ \pm}\right)=\delta_{i, j}, \quad\left(l_{i j}^{ \pm}\right)^{*}=S\left(l_{j i}^{\mp}\right)
$$

and $S\left(L^{ \pm}\right)=\left(L^{ \pm}\right)^{-1}$, where the antipode is applied componentwise.

Define a scalar valued $2 n \times 2 n$ matrix $J^{\sigma}$, depending on an auxiliary parameter $\sigma \in \mathbb{R}$, by

$$
J^{\sigma}=\left(1-q^{2 \sigma}\right) \sum_{k=1}^{n} E_{k, k}-q^{\sigma} \sum_{k=1}^{2 n} E_{k, 2 n+1-k} .
$$

The matrix $J^{\sigma}$ is a solution of a reflection equation, see [NDS97, Proposition 2.2] or [DS99, $\S 6$ ] for details. Observe, furthermore, that $J^{\sigma}$ is invertible, with inverse

$$
\left(J^{\sigma}\right)^{-1}=\left(1-q^{-2 \sigma}\right) \sum_{k=1}^{n} E_{2 n+1-k, 2 n+1-k}-q^{-\sigma} \sum_{k=1}^{2 n} E_{k, 2 n+1-k} .
$$

Definition 3.1 [NDS97, DS99]. Let $\mathfrak{k}_{\sigma} \subset U_{q}(\mathfrak{g})$ be the linear subspace spanned by the $4 n^{2}$ matrix coefficients of $L^{+} J^{\sigma}-J^{\sigma} L^{-}$.

Observe that $\mathfrak{k}_{\sigma} \subset U_{q}(\mathfrak{g})$ is a two-sided coideal, i.e. $\Delta\left(\mathfrak{k}_{\sigma}\right) \subseteq \mathfrak{k}_{\sigma} \otimes U_{q}(\mathfrak{g})+U_{q}(\mathfrak{g}) \otimes \mathfrak{k}_{\sigma}$ and $\epsilon\left(\mathfrak{k}_{\sigma}\right)=0$. Note, furthermore, that $\mathfrak{k}_{\sigma}^{*}=S\left(\mathfrak{k}_{\sigma}\right)$ since $J^{\sigma}$ is symmetric, so $\mathfrak{k}_{\sigma}$ is not $*$-stable but $\mathfrak{k}_{\sigma}$ is $\omega$-stable, where $\omega: U_{q}(\mathfrak{g}) \rightarrow U_{q}(\mathfrak{g})$ is the involutive, anti-linear algebra isomorphism $\omega=* \circ S$. Note that the involution $\omega$ is related to the $*$-structure on $\mathbb{C}_{q}[G]$ by

$$
f^{*}(X)=\overline{f(\omega(X))}, \quad f \in \mathbb{C}_{q}[G], X \in U_{q}(\mathfrak{g}) .
$$

It is convenient to write

$$
\omega_{\delta}(X)=K^{-\delta} \omega(X) K^{\delta}, \quad X \in U_{q}(\mathfrak{g}) .
$$

Note that $\omega_{\delta}$ is an anti-linear algebra involution of $U_{q}(\mathfrak{g})$, which acts on the algebraic generators of $U_{q}(\mathfrak{g})$ by

$$
\omega_{\delta}\left(K_{i}^{ \pm 1}\right)=K_{i}^{\mp 1}, \quad \omega_{\delta}\left(x_{j}\right)=-y_{j}, \quad \omega_{\delta}\left(y_{j}\right)=-x_{j}
$$

for $i=1, \ldots, 2 n$ and $j=1, \ldots, 2 n-1$.

For a subspace $U \subset U_{q}(\mathfrak{g})$, let $I(U) \subseteq U_{q}(\mathfrak{g})$ be the left ideal of $U_{q}(\mathfrak{g})$ generated by $U$ and let $A(U) \subseteq U_{q}(\mathfrak{g})$ be the unital subalgebra generated by $I(U)$. Applied to $\mathfrak{k}_{\sigma}$ and $\mathfrak{k}_{\sigma}^{*}$, we thus obtain two unital subalgebras $A\left(\mathfrak{k}_{\sigma}\right)$ and $A\left(\mathfrak{k}_{\sigma}^{*}\right)$ of $U_{q}(\mathfrak{g})$. In the following lemma we link the algebras $A\left(\mathfrak{k}_{\sigma}\right)$ and $A\left(\mathfrak{k}_{\sigma}^{*}\right)$ to $\mathcal{A}_{\sigma}$.

LEMMA 3.2.

(i) $\mathcal{A}_{\sigma} \cup \omega\left(\mathcal{A}_{\sigma}\right) \subseteq A\left(\mathfrak{k}_{\sigma}\right)$.

(ii) $\omega_{\delta}\left(\mathcal{A}_{\sigma}\right) \subseteq A\left(\mathfrak{k}_{\sigma}^{*}\right)$.

Proof. (i) Note that $A\left(\mathfrak{k}_{\sigma}\right)$ is $\omega$-stable, because $\mathfrak{k}_{\sigma}$ is $\omega$-stable. It thus suffices to prove that $\mathcal{A}_{\sigma} \subseteq$ $A\left(\mathfrak{k}_{\sigma}\right)$. 


\section{A. A. Oblomkov and J. V. Stokman}

The left ideal $I\left(\mathfrak{k}_{\sigma}\right)$ contains the matrix coefficients of the $U_{q}(\mathfrak{g})$-valued matrix

$$
\left(t_{i, j}\right)_{i, j}=S\left(L^{+}\right)\left(L^{+} J^{\sigma}-J^{\sigma} L^{-}\right)=J^{\sigma}-S\left(L^{+}\right) J^{\sigma} L^{-} .
$$

By direct computations, one can verify that

$$
\begin{gathered}
t_{i, 2 n+1-i}=-q^{\sigma}+q^{\sigma} C_{i}^{-1}, \quad t_{j, 2 n-j}=q^{\sigma}\left(q^{-1}-q\right) B_{j}, \\
t_{n, n}=q^{\sigma}\left(q^{-1}-q\right) B_{n}^{\sigma}+1-q^{2 \sigma}
\end{gathered}
$$

for $i \in\{1, \ldots, n\}$ and $j \in\{1, \ldots, 2 n-1\} \backslash\{n\}$. The left ideal $I\left(\mathfrak{k}_{\sigma}\right)$ also contains the matrix coefficients of

$$
S\left(L^{-}\right)\left(J^{\sigma}\right)^{-1}\left(L^{+} J^{\sigma}-J^{\sigma} L^{-}\right)\left(J^{\sigma}\right)^{-1}=S\left(L^{-}\right)\left(J^{\sigma}\right)^{-1} L^{+}-\left(J^{\sigma}\right)^{-1} .
$$

Computing the coefficients of the antidiagonal shows that $q^{-\sigma}-q^{-\sigma} C_{i} \in I\left(\mathfrak{t}_{\sigma}\right)$ for $i \in\{1, \ldots, n\}$. Consequently, all algebraic generators of $\mathcal{A}_{\sigma}$ are contained in $A\left(\mathfrak{k}_{\sigma}\right)$, and hence $\mathcal{A}_{\sigma} \subseteq A\left(\mathfrak{k}_{\sigma}\right)$.

(ii) Observe that

$$
\begin{aligned}
\omega_{\delta}\left(C_{i}^{ \pm 1}\right) & =C_{i}^{\mp 1}, \\
\omega_{\delta}\left(B_{j}\right) & =-q\left(K_{2 n-j} y_{2 n-j} K_{j}+K_{2 n-j} K_{j+1} x_{j}\right), \\
\omega_{\delta}\left(B_{n}^{\sigma}\right) & =-\left(K_{n} y_{n} K_{n}+K_{n} K_{n+1} x_{n}+\left(\frac{q^{\sigma}-q^{-\sigma}}{q-q^{-1}}\right) K_{n}^{2}\right),
\end{aligned}
$$

for $i \in\{1, \ldots, n\}$ and $j \in\{1, \ldots, 2 n-1\} \backslash\{n\}$, are algebraic generators of $\omega_{\delta}\left(\mathcal{A}_{\sigma}\right)$. Using similar arguments as in the proof of (i), it can be shown that all these generators are contained in $A\left(\mathfrak{k}_{\sigma}^{*}\right)=$ $A\left(S\left(\mathfrak{k}_{\sigma}\right)\right)$.

For a left $U_{q}(\mathfrak{g})$-module $M$ and a unital subalgebra $A \subset U_{q}(\mathfrak{g})$ we define the subspace of $A$-invariant vectors in $M$ by

$$
M^{A}=\{m \in M \mid a m=\epsilon(a) m, \forall a \in A\} .
$$

Lemma 3.3. For the $U_{q}(\mathfrak{g})$-subalgebras $A=\mathcal{A}_{\sigma}, A=\omega\left(\mathcal{A}_{\sigma}\right)$ and $A=\omega_{\delta}\left(\mathcal{A}_{\sigma}\right)$, we have

(i) $\operatorname{Dim}\left(L_{\lambda}^{A}\right)=0$ if $\lambda \in P_{2 n}^{+} \backslash\left(\Lambda_{n}^{+}\right)^{\natural}$;

(ii) $\operatorname{Dim}\left(L_{\lambda}^{A}\right) \leqslant 1$ if $\lambda \in\left(\Lambda_{n}^{+}\right)^{\natural}$.

Proof. The statement for $A=\mathcal{A}_{\sigma}$ follows from the results in $\S 2$.

For $\omega\left(\mathcal{A}_{\sigma}\right)$ and $\omega_{\delta}\left(\mathcal{A}_{\sigma}\right)$, we first remark that any simple, finite-dimensional $U_{q}(\mathfrak{g})$-module $L_{\lambda}$ $\left(\lambda \in P_{2 n}^{+}\right)$is semisimple as module over the subalgebras $\omega\left(\mathcal{A}_{\sigma}\right)$ and $\omega_{\delta}\left(\mathcal{A}_{\sigma}\right)$. Clearly it suffices to prove this for $\omega_{\delta}\left(\mathcal{A}_{\sigma}\right)$. In this case we first note that

$$
\omega_{\delta}\left(X^{*}\right)=\left(\omega_{\delta}(X)\right)^{*}, \quad X \in U_{q}(\mathfrak{g}),
$$

which follows easily from the fact that

$$
S^{2}(X)=K^{-2 \delta} X K^{2 \delta}, \quad X \in U_{q}(\mathfrak{g}) .
$$

We conclude that $\omega_{\delta}\left(\mathcal{A}_{\sigma}\right)$ is a $*$-subalgebra of $U_{q}(\mathfrak{g})$, and hence $L_{\lambda}$ is $\omega_{\delta}\left(\mathcal{A}_{\sigma}\right)$-semisimple.

Straightforward adjustments of the arguments in $\S 2$ now lead to the construction of expectation values and to the analogue of Proposition 2.2 for the algebras $\omega\left(\mathcal{A}_{\sigma}\right)$ and $\omega_{\delta}\left(\mathcal{A}_{\sigma}\right)$. Since the elements $C_{i}(i=1, \ldots, n)$ are in $\omega\left(\mathcal{A}_{\sigma}\right)$ and $\omega_{\delta}\left(\mathcal{A}_{\sigma}\right)$, the lemma now follows for these two subalgebras in the same way as for $\mathcal{A}_{\sigma}$.

If the algebra $A \subseteq U_{q}(\mathfrak{g})$ is of the form $A=A(U)$ for some vector space $U \subset U_{q}(\mathfrak{g})$ and $\left.\epsilon\right|_{U} \equiv 0$, then $M^{A(U)}$ consists of the vectors $m \in M$ which are annihilated by $u \in U$. In this situation, $M^{A(U)}$ is called the subspace of $U$-fixed vectors, cf. [NDS97, DS99]. In particular, this applies to $U=\mathfrak{k}_{\sigma}$ and $U=\mathfrak{k}_{\sigma}^{*}$. We now recall the following result (see [NDS97, Theorem 2.6] and [DS99, Theorem 6.6]). 


\section{SPHERICAL FUNCTIONS AND MACDONALD-KOORNWINDER POLYNOMIALS}

Proposition 3.4. For $U=\mathfrak{k}_{\sigma}$ and $U=\mathfrak{k}_{\sigma}^{*}$ we have

$$
\operatorname{Dim}\left(L_{\lambda}^{A(U)}\right)= \begin{cases}0 & \text { if } \lambda \in P_{2 n}^{+} \backslash\left(\Lambda_{n}^{+}\right)^{\natural}, \\ 1 & \text { if } \lambda \in\left(\Lambda_{n}^{+}\right)^{\natural} .\end{cases}
$$

Combined with Lemma 3.2 and Lemma 3.3, we obtain the following.

Lemma 3.5. For $\lambda \in P_{2 n}^{+}$we have:

(i) $L_{\lambda}^{\mathcal{A}_{\sigma}}=L_{\lambda}^{\omega\left(\mathcal{A}_{\sigma}\right)}=L_{\lambda}^{A\left(\mathfrak{k}_{\sigma}\right)}$;

(ii) $L_{\lambda}^{\omega_{\delta}\left(\mathcal{A}_{\sigma}\right)}=L_{\lambda}^{A\left(\mathfrak{k}_{\sigma}^{*}\right)}$.

Proof. By Lemma 3.3 and Proposition 3.4, all spaces in (i) and (ii) are $\{0\}$ when $\lambda \in P_{2 n}^{+} \backslash\left(\Lambda_{n}^{+}\right)^{\natural}$.

Let $\lambda \in\left(\Lambda_{n}^{+}\right)^{\natural}$. Lemma 3.2 implies $L_{\lambda}^{\mathcal{A}_{\sigma}} \supseteq L_{\lambda}^{A\left(\mathfrak{k}_{\sigma}\right)}, L_{\lambda}^{\omega\left(\mathcal{A}_{\sigma}\right)} \supseteq L_{\lambda}^{A\left(\mathfrak{k}_{\sigma}\right)}$ and $L_{\lambda}^{\omega_{\delta}\left(\mathcal{A}_{\sigma}\right)} \supseteq L_{\lambda}^{A\left(\mathfrak{k}_{\sigma}^{*}\right)}$. Since $\operatorname{Dim}\left(L_{\lambda}^{A}\right)=1$ for $A=A\left(\mathfrak{k}_{\sigma}\right)$ and $A=A\left(\mathfrak{k}_{\sigma}^{*}\right)$ by Proposition 3.4, we conclude from Lemma 3.3 that $L_{\lambda}^{\mathcal{A}_{\sigma}}=L_{\lambda}^{A\left(\mathfrak{k}_{\sigma}\right)}, L_{\lambda}^{\omega\left(\mathcal{A}_{\sigma}\right)}=L_{\lambda}^{A\left(\mathfrak{k}_{\sigma}\right)}$ and $L_{\lambda}^{\omega_{\delta}\left(\mathcal{A}_{\sigma}\right)}=L_{\lambda}^{A\left(\mathfrak{k}_{\sigma}^{*}\right)}$.

The following result is a direct consequence of the definition of $\omega_{\delta}$ and of Lemma 3.5.

Corollary 3.6. $L_{\lambda}^{A\left(\mathfrak{k}_{\sigma}^{*}\right)}=K^{-\delta} L_{\lambda}^{A\left(\mathfrak{k}_{\sigma}\right)}$ for all $\lambda \in P_{2 n}^{+}$.

The main object of study in [NDS97] and [DS99] is the space of $\left(\mathfrak{k}_{\sigma}, \mathfrak{k}_{\tau}\right)$-fixed regular functions on $U_{q}(\mathfrak{g})$, which is defined as follows.

Definition 3.7. The space of $\left(\mathfrak{k}_{\sigma}, \mathfrak{k}_{\tau}\right)$-fixed functions on $U_{q}(\mathfrak{g})$ is defined by

$$
\mathcal{H}^{\sigma, \tau}=\left\{f \in \mathbb{C}_{q}[G] \mid Y \cdot f=f \cdot Z=0, \forall Y \in \mathfrak{k}_{\sigma}, \forall Z \in \mathfrak{k}_{\tau}\right\} .
$$

Proposition 3.8. The subspaces $\mathcal{H}^{\sigma, \tau}$ and $F_{\overrightarrow{0}}^{\sigma, \tau}$ of $\mathbb{C}_{q}[G]$ are related by

$$
\mathcal{H}^{\sigma, \tau}=F_{\overrightarrow{0}}^{\sigma, \tau} \cdot K^{-\delta} \text {. }
$$

Proof. Any $f \in \mathcal{H}^{\sigma, \tau}$ can be written as a linear combination of functions

$$
U_{q}(\mathfrak{g}) \ni X \mapsto\left\langle X v_{\sigma}, \widetilde{v}_{\tau}\right\rangle_{\lambda}
$$

with $v_{\sigma} \in L_{\lambda}^{A\left(\mathfrak{k}_{\sigma}\right)}, \widetilde{v}_{\tau} \in L_{\lambda}^{A\left(\mathfrak{k}_{\tau}^{*}\right)}$ and $\lambda \in\left(\Lambda_{n}^{+}\right)^{\natural}$. On the other hand, any $g \in F_{\overrightarrow{0}}^{\sigma, \tau}$ can be written as a linear combination of functions

$$
U_{q}(\mathfrak{g}) \ni X \mapsto\left\langle X v_{\sigma}, \widehat{v}_{\tau}\right\rangle_{\lambda}
$$

with $v_{\sigma} \in L_{\lambda}^{\mathcal{A}_{\sigma}}, \widehat{v}_{\tau} \in L_{\lambda}^{\mathcal{A}_{\tau}}$ and $\lambda \in\left(\Lambda_{n}^{+}\right)^{\natural}$ since $\rho(0,0)_{\sigma}=\rho(0)_{\sigma}=\left.\epsilon\right|_{\mathcal{A}_{\sigma}}$. The proposition now follows from the fact that $L_{\lambda}^{A\left(\mathfrak{k}_{\sigma}\right)}=L_{\lambda}^{\mathcal{A}_{\sigma}}, L_{\lambda}^{A\left(\mathfrak{k}_{\tau}^{*}\right)}=K^{-\delta} L_{\lambda}^{A\left(\mathfrak{k}_{\tau}\right)}=K^{-\delta} L_{\lambda}^{\mathcal{A}_{\tau}}$ and from the fact that $K^{-\delta}$ is $*$-self-adjoint.

Remark 3.9 .

(i) The fact that $\mathfrak{k}_{\sigma}$ and $\mathfrak{k}_{\tau}$ are $\omega$-stable two-sided coideals implies that $\mathcal{H}^{\sigma, \tau} \subseteq \mathbb{C}_{q}[G]$ is a unital *-subalgebra, cf. [NDS97, DS99]. Furthermore, $K^{-\delta} \in U_{q}(\mathfrak{g})$ is a group-like element, so the previous proposition shows that $F_{\overrightarrow{0}}^{\sigma, \tau} \subseteq \mathbb{C}_{q}[G]$ is a unital subalgebra (but it is not *-stable!).

(ii) Observe that $\epsilon\left(\omega_{\delta}(X)\right)=\overline{\epsilon(X)}$ for $X \in U_{q}(\mathfrak{g})$. Combined with Lemma 3.5, the proof of Proposition 3.8 and (3.5), this leads to the alternative description

$$
\mathcal{H}^{\sigma, \tau}=\left\{f \in \mathbb{C}_{q}[G] \mid f\left(\omega_{\delta}(b) X a\right)=\epsilon(a) \overline{\epsilon(b)} f(X), \forall X \in U_{q}(\mathfrak{g}), \forall a \in \mathcal{A}_{\sigma}, \forall b \in \mathcal{A}_{\tau}\right\}
$$

for the space of $\left(\mathfrak{k}_{\sigma}, \mathfrak{k}_{\tau}\right)$-fixed regular functions on $U_{q}(\mathfrak{g})$. 


\section{A. A. Oblomkov and J. V. Stokman}

Recall that the restriction map $\left.\right|_{T}: F_{\overrightarrow{0}}^{\sigma, \tau} \rightarrow \mathbb{C}\left[u^{ \pm 1}\right]$ involved a $\delta$-shift: $\left.f\right|_{T}\left(q^{\lambda}\right)=f\left(K^{\lambda-\delta}\right)$ for all $\lambda \in P_{2 n}$. When dealing with the $*$-subalgebra $\mathcal{H}^{\sigma, \tau}$, it is convenient to use the restriction map without $\delta$-shift,

$$
\operatorname{Res}_{T}: \mathcal{H}^{\sigma, \tau} \rightarrow \mathbb{C}\left[u^{ \pm 1}\right], \quad \operatorname{Res}_{T}(f)\left(q^{\lambda}\right)=f\left(K^{\lambda}\right), \quad \forall \lambda \in P_{2 n},
$$

since then we have

$$
\operatorname{Res}_{T}\left(f \cdot K^{-\delta}\right)=\left.f\right|_{T}, \quad \forall f \in F_{\overrightarrow{0}}^{\sigma, \tau}
$$

cf. Proposition 3.8. Note that $\operatorname{Res}_{T}$ is a $*$-algebra homomorphism, with the $*$-structure on $\mathbb{C}\left[u^{ \pm 1}\right]$ defined by

$$
p^{*}=\sum_{\mu \in \Lambda_{n}} \overline{c_{\mu}} u^{-\mu} \quad \text { if } p=\sum_{\mu \in \Lambda_{n}} c_{\mu} u^{\mu} \in \mathbb{C}\left[u^{ \pm 1}\right] .
$$

We are now in a position to translate the main result from [NDS97] to our present setup.

Proposition 3.10. Theorem 1.21 is valid for $\vec{\kappa}=\overrightarrow{0}$.

Proof. Observe that $\delta(0,0)=0$; hence we can take the unit $1 \in \mathbb{C}_{q}[G]$ as nonzero element in $F_{\overrightarrow{0}}^{\sigma, \tau}(0)$. Clearly $\left.1\right|_{T}$ is the unit of $\mathbb{C}\left[u^{ \pm 1}\right]$. This gives Theorem 1.21 (ii) for $\vec{\kappa}=\overrightarrow{0}$.

The generalized Chevalley restriction theorem (Theorem 1.21(i)) for $\vec{\kappa}=\overrightarrow{0}$ reduces to the statement that $\left.\right|_{T}: F_{\overrightarrow{0}}^{\sigma, \tau} \rightarrow \mathbb{C}\left[u^{ \pm 1}\right]^{W}$ is a linear isomorphism. In view of Proposition 3.8 and the $\delta$-shift in the definition of the restriction map $\left.\right|_{T}$, this is equivalent to the statement that $\operatorname{Res}_{T}: \mathcal{H}^{\sigma, \tau} \rightarrow \mathbb{C}\left[u^{ \pm 1}\right]^{W}$ is a linear isomorphism, which follows from [NDS97, Theorem 3.2], see also [DS99, Theorem 7.5]. For a direct proof of this result for an arbitrary quantum symmetric pair, see [Let03].

Let $0 \neq f_{\mu} \in F_{\overrightarrow{0}}^{\sigma, \tau}(\mu)$ be an elementary spherical function of degree $\mu \in \Lambda_{n}^{+}$, then $f_{\mu} \cdot K^{-\delta} \in$ $\mathcal{H}^{\sigma, \tau} \cap W\left(\mu^{\natural}\right)$. Consequently, [NDS97, Theorem 3.4] shows that the restriction $\left.f_{\mu}\right|_{T}=\operatorname{Res}_{T}\left(f_{\mu}\right.$. $\left.K^{-\delta}\right) \in \mathbb{C}\left[u^{ \pm 1}\right]^{W}$ is a nonzero scalar multiple of the Macdonald-Koornwinder polynomial

$$
P_{\mu}^{\sigma, \tau}(u)=P_{\mu}\left(u ;-q^{\sigma+\tau+1},-q^{-\sigma-\tau+1}, q^{\sigma-\tau+1}, q^{-\sigma+\tau+1} ; q^{2}, q^{2}\right),
$$

(see also [DS99, Theorem 7.5]). This proves Theorem 1.21(iii) for $\vec{\kappa}=\overrightarrow{0}$.

\section{The generalized Chevalley theorem}

In this section we prove the generalized Chevalley restriction theorem (see Theorem 1.21(i)).

To simplify the notation, we write $[\cdot, \cdot]_{\mu}$ for the scalar product $\langle\cdot, \cdot\rangle_{\left(\mu+\delta\left(\kappa, \kappa_{1}\right)\right)^{\natural}}$ on the $U_{q}(\mathfrak{g})$ module $L_{\left(\mu+\delta\left(\kappa, \kappa_{1}\right)\right)^{\natural}}\left(\mu \in \Lambda_{n}^{+}\right)$.

Lemma 4.1. Let $\mu \in \Lambda_{n}^{+}$and identify $V\left(\kappa, \kappa_{1}\right)_{\tau}$ with its unique copy in the semisimple $\mathcal{A}_{\tau}$-module $L_{\left(\mu+\delta\left(\kappa, \kappa_{1}\right)\right)^{\natural}}^{\tau}$ and $V\left(\kappa_{2}\right)_{\sigma}$ with its unique copy in the semisimple $\mathcal{A}_{\sigma}$-module $L_{\left(\mu+\delta\left(\kappa, \kappa_{1}\right)\right)^{\natural}}^{\sigma}$. Let $f_{\mu} \in$ $F_{\vec{\kappa}}^{\sigma, \tau}(\mu)$ be an elementary vector valued spherical function of degree $\mu$. Then

$$
\left.f_{\mu}\right|_{T}\left(q^{\lambda}\right)=\left[K^{\lambda-\delta} v, w\right]_{\mu}, \quad \forall \lambda \in P_{2 n}
$$

for suitably normalized vectors $0 \neq v \in V\left(\kappa_{2}\right)_{\sigma}$ and $0 \neq w \in \widetilde{V}\left(\kappa, \kappa_{1}\right)_{\tau}$. Furthermore,

$$
\left.f_{\mu}\right|_{T}=\sum_{-\mu-\delta\left(\kappa, \kappa_{1}\right) \leqslant \nu \leqslant \mu+\delta\left(\kappa, \kappa_{1}\right)} c_{\nu} u^{\nu} \in \mathbb{C}\left[u^{ \pm 1}\right]
$$

for certain constants $c_{\nu} \in \mathbb{C}\left(\nu \in \Lambda_{n}\right)$, and $c_{ \pm \mu \pm \delta\left(\kappa, \kappa_{1}\right)} \neq 0$.

Proof. Choose an orthonormal basis $\left\{b_{1}, \ldots, b_{m}\right\}$ of $V\left(\kappa, \kappa_{1}\right)_{\tau}$ with respect to the restriction of $[\cdot, \cdot]_{\mu}$ to $V\left(\kappa, \kappa_{1}\right)_{\tau}$ such that $b_{1} \in \widetilde{V}\left(\kappa, \kappa_{1}\right)_{\tau}$. In particular, $b_{1}$ is a nonzero constant multiple of $w$. 


\section{SPHERICAL FUnCTIONS AND MACDONALD-KOORNWINDER POLYNOMIALS}

Then $f_{\mu} \in F_{\vec{\kappa}}^{\sigma, \tau}(\mu)$ can be realized as

$$
f_{\mu}(X)=\sum_{i=1}^{m}\left[X v, b_{i}\right]_{\mu} b_{i}, \quad X \in U_{q}(\mathfrak{g})
$$

for some $0 \neq v \in V\left(\kappa_{2}\right)_{\sigma}$. We have $f_{\mu}(X) \in \widetilde{V}\left(\kappa, \kappa_{1}\right)_{\tau}$ for $X \in U^{0}$, see (the proof of) Lemma 1.20. Using the identification $\widetilde{V}\left(\kappa, \kappa_{1}\right)_{\tau} \simeq \mathbb{C}$ of vector spaces given by the assignment $b_{1} \mapsto 1$, we obtain

$$
\left.f_{\mu}\right|_{T}\left(q^{\lambda}\right)=f_{\mu}\left(K^{\lambda-\delta}\right)=\left[K^{\lambda-\delta} v, b_{1}\right]_{\mu}, \quad \forall \lambda \in P_{2 n} .
$$

Now both vectors $v$ and $b_{1}$ satisfy $C_{i} v=v$ and $C_{i} b_{1}=b_{1}$ for $i=1, \ldots, n$, hence $v[\lambda]=0$ and $b_{1}[\lambda]=0$ for $\lambda \in P_{2 n}$ unless $\lambda \in\left(\Lambda_{n}\right)^{\natural}$. Since $\natural: \Lambda_{n} \rightarrow\left(\Lambda_{n}\right)^{\natural} \subset P_{2 n}$ is a bijection of partially ordered sets and $(-\nu)^{\natural}=w_{0}\left(\nu^{\natural}\right)$ for $\nu \in \Lambda_{n}$, we conclude that the weight decompositions of $v$ and $b_{1}$ are of the form

$$
v=\sum_{-\mu-\delta\left(\kappa, \kappa_{1}\right) \leqslant \nu \leqslant \mu+\delta\left(\kappa, \kappa_{1}\right)} v\left[\nu^{\natural}\right], \quad b_{1}=\sum_{-\mu-\delta\left(\kappa, \kappa_{1}\right) \leqslant \nu \leqslant \mu+\delta\left(\kappa, \kappa_{1}\right)} b_{1}\left[\nu^{\natural}\right],
$$

with $v\left[\nu^{\natural}\right]$ and $b_{1}\left[\nu^{\natural}\right]$ the components of the vectors $v$ and $b_{1}$ in the weight space of the $U_{q}(\mathfrak{g})$-module $L_{\left(\mu+\delta\left(\kappa, \kappa_{1}\right)\right)^{\natural}}$ of weight $\nu^{\natural}$.

A simple application of Lemma 2.5 shows that $v\left[\left( \pm \mu \pm \delta\left(\kappa, \kappa_{1}\right)\right)^{\natural}\right] \neq 0$. Denote by $\widetilde{L}_{\left(\mu+\delta\left(\kappa, \kappa_{1}\right)\right)^{\natural}}^{\tau}$ the vectors $v \in L_{\left(\mu+\delta\left(\kappa, \kappa_{1}\right)\right)^{\natural}}^{\tau}$ satisfying $C_{i} v=v$ for $i=1, \ldots, n$. This subspace contains the onedimensional weight spaces $L_{\left(\mu+\delta\left(\kappa, \kappa_{1}\right)\right)^{\natural}}\left[\left( \pm \mu \pm \delta\left(\kappa, \kappa_{1}\right)\right)^{\natural}\right]$ and $\widetilde{L}_{\left(\mu+\delta\left(\kappa, \kappa_{1}\right)\right)^{\natural}}^{\tau} \cap \widetilde{V}\left(\kappa, \kappa_{1}\right)_{\tau}=\mathbb{C}\left\{b_{1}\right\}$. Lemma 2.5 implies $b_{1}\left[\left( \pm \mu \pm \delta\left(\kappa, \kappa_{1}\right)\right)^{\natural}\right] \neq 0$. Hence

$$
\begin{aligned}
\left.f_{\mu}\right|_{T} & =\sum_{-\mu-\delta\left(\kappa, \kappa_{1}\right) \leqslant \nu \leqslant \mu+\delta\left(\kappa, \kappa_{1}\right)}\left[K^{-\delta} v\left[\nu^{\natural}\right], b_{1}\left[\nu^{\natural}\right]\right]_{\mu} u^{\nu} \\
& =\sum_{-\mu-\delta\left(\kappa, \kappa_{1}\right) \leqslant \nu \leqslant \mu+\delta\left(\kappa, \kappa_{1}\right)} c_{\nu} u^{\nu}
\end{aligned}
$$

with $c_{\nu} \in \mathbb{C}$ satisfying the conditions as stated in the lemma.

A more detailed analysis of the elementary vector valued spherical functions requires the right coideal algebra structure of $\mathcal{A}_{\sigma}$. Recall that a right $U_{q}(\mathfrak{g})$-comodule $\left(M, \delta_{M}\right)$ is a vector space $M$ together with a linear map $\delta_{M}: M \rightarrow M \otimes U_{q}(\mathfrak{g})$ satisfying

$$
\left(\delta_{M} \otimes \operatorname{Id}_{U_{q}(\mathfrak{g})}\right) \circ \delta_{M}=\left(\operatorname{Id}_{M} \otimes \Delta\right) \circ \delta_{M}, \quad\left(\operatorname{Id}_{M} \otimes \epsilon\right) \circ \delta_{M}=\operatorname{Id}_{M} .
$$

If $M$ is a unital algebra and $\delta_{M}$ is a (unit preserving) algebra homomorphism, then $\left(M, \delta_{M}\right)$ is called a right $U_{q}(\mathfrak{g})$-comodule algebra. In particular, a unital subalgebra $A \subseteq U_{q}(\mathfrak{g})$ satisfying $\Delta(A) \subseteq A \otimes U_{q}(\mathfrak{g})$ gives rise to the right comodule algebra $\left(A,\left.\Delta\right|_{A}\right)$. In this case, $A$ is called a right coideal subalgebra of $U_{q}(\mathfrak{g})$.

By computing the action of the comultiplication $\Delta$ on the algebraic generators of $\mathcal{A}_{\sigma}$, one verifies that $\mathcal{A}_{\sigma} \subseteq U_{q}(\mathfrak{g})$ is a right $U_{q}(\mathfrak{g})$-coideal algebra. Using the isomorphism $\pi_{\sigma}: \mathcal{A} \rightarrow \mathcal{A}_{\sigma}$ of algebras, see Proposition $1.4, \mathcal{A}$ inherits a unique right $U_{q}(\mathfrak{g})$-comodule structure such that $\pi_{\sigma}: \mathcal{A} \rightarrow \mathcal{A}_{\sigma}$ is an isomorphism of right $U_{q}(\mathfrak{g})$-comodule algebras. The right comodule map for $\mathcal{A}$ turns out to be independent of $\sigma$, and is given as follows (cf., e.g., [Let02]).

Proposition 4.2. The algebra $\mathcal{A}$ has the structure of a right $U_{q}(\mathfrak{g})$-comodule algebra, with comodule $\operatorname{map} \delta_{\mathcal{A}}$ given explicitly by

$$
\begin{aligned}
\delta_{\mathcal{A}}\left(\gamma_{i}^{ \pm 1}\right) & =\gamma_{i}^{ \pm 1} \otimes K_{i}^{ \pm 1} K_{2 n+1-i}^{ \pm 1}, \\
\delta_{\mathcal{A}}\left(\beta_{j}\right) & =\beta_{j} \otimes K_{j}^{-1} K_{2 n-j}^{-1}+\gamma_{j+1}^{-1} \otimes y_{j} K_{j+1}^{-1} K_{2 n-j}^{-1}+\gamma_{j}^{-1} \otimes K_{j}^{-1} x_{2 n-j} K_{2 n-j}^{-1}
\end{aligned}
$$




\section{A. A. Oblomkov and J. V. Stokman}

for $i=1, \ldots, 2 n$ and $j=1, \ldots, 2 n-1$. The map $\pi_{\sigma}: \mathcal{A} \rightarrow \mathcal{A}_{\sigma}$ is an isomorphism of right $U_{q}(\mathfrak{g})$-comodule algebras.

Finite-dimensional $\mathcal{A}_{\sigma}$-modules do not form a tensor category, since $\mathcal{A}_{\sigma} \subset U_{q}(\mathfrak{g})$ is not a coalgebra. However, the right coideal algebra structure of $\mathcal{A}_{\sigma}$ implies that the tensor product $M \otimes N$ of an $\mathcal{A}_{\sigma}$-module $M$ and a $U_{q}(\mathfrak{g})$-module $N$ is an $\mathcal{A}_{\sigma}$-module by the usual formula

$$
a \cdot(m \otimes n)=\sum a_{1} \cdot m \otimes a_{2} \cdot n, \quad m \in M, n \in N,
$$

where (recall) $\Delta(a)=\sum a_{1} \otimes a_{2}$ for $a \in \mathcal{A}_{\sigma}$ with the $a_{1}$ from $\mathcal{A}_{\sigma}$. In the special case that $M$ is the one-dimensional $\mathcal{A}_{\sigma}$-module $V(k)_{\sigma}(k \in \mathbb{Z})$, we can naturally relate the $\mathcal{A}_{\sigma}$-module $V(k)_{\sigma} \otimes N$ to the $\mathcal{A}_{\sigma+2 k^{-}}$module $N^{\sigma+2 k}$ in the following manner.

Let $\chi_{k}^{\sigma}: \mathcal{A}_{\sigma} \rightarrow \mathbb{C}$ be the character of $V(k)_{\sigma}$. The $\mathcal{A}_{\sigma}$-action on $V(k)_{\sigma} \otimes N$ can then be rewritten as

$$
a \cdot(m \otimes n)=m \otimes \zeta_{k}^{\sigma}(a) \cdot n, \quad a \in \mathcal{A}_{\sigma}, m \in V(k)_{\sigma}, n \in N,
$$

with $\zeta_{k}^{\sigma}: \mathcal{A}_{\sigma} \rightarrow U_{q}(\mathfrak{g})$ the unital algebra homomorphism

$$
\zeta_{k}^{\sigma}(a)=\sum \chi_{k}^{\sigma}\left(a_{1}\right) a_{2}, \quad a \in \mathcal{A}_{\sigma} .
$$

Lemma 4.3. The map $\zeta_{k}^{\sigma}$ defines an algebra isomorphism $\zeta_{k}^{\sigma}: \mathcal{A}_{\sigma} \rightarrow \mathcal{A}_{\sigma+2 k}$, with inverse $\zeta_{-k}^{\sigma+2 k}$ : $\mathcal{A}_{\sigma+2 k} \rightarrow \mathcal{A}_{\sigma}$. Furthermore, $\zeta_{k}^{\sigma}=\left.\pi_{\sigma+2 k} \circ \pi_{\sigma}^{-1}\right|_{\mathcal{A}_{\sigma}}$ (see Proposition 1.4 for the definition of $\pi_{\sigma}$ ), which means that

$$
\zeta_{k}^{\sigma}\left(C_{i}\right)=C_{i}, \quad \zeta_{k}^{\sigma}\left(B_{j}\right)=B_{j}, \quad \zeta_{k}^{\sigma}\left(B_{n}^{\sigma}\right)=B_{n}^{\sigma+2 k}
$$

for $i \in\{1, \ldots, 2 n\}$ and $j \in\{1, \ldots, 2 n-1\} \backslash\{n\}$.

Proof. By Lemma 1.13 the values of $\chi_{k}^{\sigma}$ on the algebraic generators of $\mathcal{A}_{\sigma}$ are given by

$$
\chi_{k}^{\sigma}\left(C_{i}\right)=1, \quad \chi_{k}^{\sigma}\left(B_{j}\right)=0, \quad \chi_{k}^{\sigma}\left(B_{n}^{\sigma}\right)=\vartheta_{0}\left(q^{\sigma+2 k}\right)
$$

for $i \in\{1, \ldots, 2 n\}$ and $j \in\{1, \ldots, 2 n-1\} \backslash\{n\}$, with $\vartheta_{l}(s)(l \in \mathbb{Z})$ defined by

$$
\vartheta_{l}(s)=\frac{s^{-1}-s q^{-2 l}}{q-q^{-1}} .
$$

Combined with Proposition 4.2 one can now easily derive (4.2). The remaining statements follow immediately.

Corollary 4.4. Let $N$ be a finite-dimensional $U_{q}(\mathfrak{g})$-module and suppose that $N^{\prime} \subset N^{\sigma+2 k}$ is an $\mathcal{A}_{\sigma+2 k}$-submodule.

(i) $V(k)_{\sigma} \otimes N^{\prime}$ is an $\mathcal{A}_{\sigma}$-submodule of $V(k)_{\sigma} \otimes N$.

(ii) If $N^{\prime} \simeq V\left(\kappa, \kappa_{1}\right)_{\sigma+2 k}$, then $V(k)_{\sigma} \otimes N^{\prime} \simeq V\left(\kappa, \kappa_{1}+k\right)_{\sigma}$ as $\mathcal{A}_{\sigma}$-modules.

Proof. (i) follows from Lemma 4.3, and (ii) follows from Lemma 4.3 and Lemma 1.13.

As a first application of these tensor product constructions we derive (a refinement of) the generalized Chevalley restriction theorem (see Theorem 1.21(i)). Before stating the result, we first recall some basic facts on tensor products of finite-dimensional $U_{q}(\mathfrak{g})$-modules which we need in the proof. For any $\lambda, \mu \in P_{2 n}^{+}$, the irreducible decomposition of the finite-dimensional $U_{q}(\mathfrak{g})$-module $L_{\lambda} \otimes L_{\mu}$ is of the form

$$
L_{\lambda} \otimes L_{\mu}=L_{\lambda+\mu} \oplus \bigoplus_{\substack{\nu \in P_{2 n}^{+} \\ \nu \prec \lambda+\mu}} L_{\nu}^{\oplus d_{\nu}^{\lambda, \mu}}
$$




\section{SPHERICAL FUNCTIONS AND MACDONALD-KOORNWINDER POLYNOMIALS}

for certain multiplicities $d_{\nu}^{\lambda, \mu} \in \mathbb{Z}_{\geqslant 0}$. The copy $L_{\lambda+\mu}$ has $v_{\lambda} \otimes v_{\mu}$ as highest weight vector. We write $\operatorname{pr}_{\lambda, \mu}: L_{\lambda} \otimes L_{\mu} \rightarrow L_{\lambda+\mu}$ for the projection along the direct sum decomposition (4.4), and $\operatorname{pr}_{\mu}=\operatorname{pr}_{\mu^{\natural}, \delta\left(\kappa, \kappa_{1}\right)^{\natural}}$ for $\mu \in \Lambda_{n}^{+}$.

Proposition 4.5. Let $\mu \in \Lambda_{n}^{+}$and choose elementary vector valued spherical functions $f_{\mu} \in F_{\vec{\kappa}}^{\sigma, \tau}(\mu)$ and $f_{0} \in F_{\vec{\kappa}}^{\sigma, \tau}(0)$. Then $\left.f_{\mu}\right|_{T}$ is divisible by $\left.f_{0}\right|_{T}$ in $\mathbb{C}\left[u^{ \pm 1}\right]$, and

$$
\frac{\left.f_{\mu}\right|_{T}}{\left.f_{0}\right|_{T}}=\sum_{\substack{\nu \in \Lambda_{n}^{+} \\ \nu \leqslant \mu}} c_{\nu} m_{\nu} \in \mathbb{C}\left[u^{ \pm 1}\right]^{W}
$$

for some constants $c_{\nu} \in \mathbb{C}$ with $c_{\mu} \neq 0$.

Proof. The proof is by induction on $\mu \in \Lambda_{n}^{+}$along the dominance order $\leqslant$. For $\mu=0$ the proposition is trivial. We identify $V(0)_{\sigma}$ with its unique copy in $L_{\mu^{\natural}}^{\sigma}$ and $V\left(\kappa_{2}\right)_{\sigma}$ with its unique copy in $L_{\delta\left(\kappa, \kappa_{1}\right)^{\natural}}^{\sigma}$, and we choose nonzero vectors

$$
w_{\sigma} \in V(0)_{\sigma} \subset L_{\mu^{\natural}}^{\sigma}, \quad u_{\sigma} \in V\left(\kappa_{2}\right)_{\sigma} \subset L_{\delta\left(\kappa, \kappa_{1}\right)^{\natural}}^{\sigma} \cdot
$$

We, furthermore, fix nonzero intertwiners

$$
\phi_{\mu} \in \operatorname{Hom}_{\mathcal{A}_{\tau}}\left(L_{\mu^{\natural}}^{\tau}, V(0)_{\tau}\right), \quad \phi_{0} \in \operatorname{Hom}_{\mathcal{A}_{\tau}}\left(L_{\delta\left(\kappa, \kappa_{1}\right)^{\natural}}^{\tau}, V\left(\kappa, \kappa_{1}\right)_{\tau}\right)
$$

and we identify $V(0)_{\tau} \simeq \mathbb{C}$ as vector spaces. Then

$$
g_{\mu}(X)=\phi_{\mu}\left(X w_{\sigma}\right), \quad f_{0}(X)=\phi_{0}\left(X u_{\sigma}\right)
$$

for $X \in U_{q}(\mathfrak{g})$ define elementary vector valued spherical functions $g_{\mu} \in F_{\overrightarrow{0}}^{\sigma, \tau}(\mu)$ and $f_{0} \in F_{\vec{\kappa}}^{\sigma, \tau}(0)$. Furthermore, $\left.g_{\mu}\right|_{T}$ is of the form

$$
\left.g_{\mu}\right|_{T}=\sum_{\substack{\nu \in \Lambda_{n}^{+} \\ \nu \leqslant \mu}} d_{\nu} m_{\nu}
$$

for some constants $d_{\nu} \in \mathbb{C}$ with $d_{\mu} \neq 0$ in view of Proposition 3.10 (in fact, $\left.g_{\mu}\right|_{T}$ is a nonzero constant multiple of a Macdonald-Koornwinder polynomial of degree $\mu$ ).

We now consider the linear map $f: U_{q}(\mathfrak{g}) \rightarrow V\left(\kappa, \kappa_{1}\right)_{\tau}$ defined by

$$
f(X)=\phi\left(X\left(w_{\sigma} \otimes u_{\sigma}\right)\right), \quad X \in U_{q}(\mathfrak{g}),
$$

with $\phi: L_{\mu^{\natural}} \otimes L_{\delta\left(\kappa, \kappa_{1}\right)^{\natural}} \rightarrow V\left(\kappa, \kappa_{1}\right)_{\tau}$ the linear map defined by

$$
\phi(u \otimes v)=\phi_{\mu}(u) \phi_{0}(v), \quad u \in L_{\mu^{\natural}}, v \in L_{\delta\left(\kappa, \kappa_{1}\right)^{\natural}} .
$$

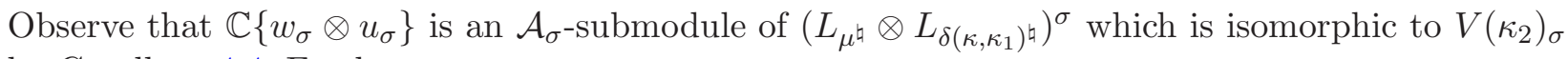
by Corollary 4.4. Furthermore,

$$
\phi \in \operatorname{Hom}_{\mathcal{A}_{\tau}}\left(\left(L_{\mu^{\natural}} \otimes L_{\delta\left(\kappa, \kappa_{1}\right)^{\natural}}\right)^{\tau}, V\left(\kappa, \kappa_{1}\right)_{\tau}\right),
$$

since

$$
\begin{aligned}
\phi(a(v \otimes w)) & =\sum \phi_{\mu}\left(a_{1} v\right) \phi_{0}\left(a_{2} w\right) \\
& =\phi_{\mu}(v) \phi_{0}\left(\zeta_{0}^{\tau}(a) w\right) \\
& =\rho\left(\kappa, \kappa_{1}\right)_{\tau}(a) \phi(v \otimes w)
\end{aligned}
$$

for $a \in \mathcal{A}_{\tau}, v \in L_{\mu^{\natural}}$ and $w \in L_{\delta\left(\kappa, \kappa_{1}\right)^{\natural}}$, where, besides the intertwining properties of $\phi_{\mu}$ and $\phi_{0}$, we have used the fact that $\zeta_{0}^{\tau}=\operatorname{Id}_{\mathcal{A}_{\tau}}$. Hence we conclude that $f \in F_{\vec{\kappa}}^{\sigma, \tau}$.

By (4.4) and Corollary 1.18 we have an expansion

$$
f=\sum_{\substack{\nu^{\prime} \in \Lambda_{n}^{+} \\ \nu^{\prime} \leqslant \mu}} f_{\nu^{\prime}}
$$




\section{A. A. Oblomkov and J. V. Stokman}

with $f_{\nu^{\prime}} \in F_{\vec{\kappa}}^{\sigma, \tau}\left(\nu^{\prime}\right)$ and $f_{\mu}(\cdot)=\phi\left(\cdot \operatorname{pr}_{\mu}\left(w_{\sigma} \otimes u_{\sigma}\right)\right)$. Lemma 2.5 implies that $\operatorname{pr}_{\mu}\left(w_{\sigma} \otimes u_{\sigma}\right)$ spans the unique copy of $V\left(\kappa_{2}\right)_{\sigma}$ in $L_{\left(\mu+\delta\left(\kappa, \kappa_{1}\right)\right)^{\natural}}^{\sigma}$. Furthermore, $\phi \circ \operatorname{pr}_{\mu} \neq 0$ since

$$
\phi\left(v_{\mu^{\natural}} \otimes v_{\delta\left(\kappa, \kappa_{1}\right)^{\natural}}\right)=\phi_{\mu}\left(v_{\mu^{\natural}}\right) \phi_{0}\left(v_{\delta\left(\kappa, \kappa_{1}\right)^{\natural}}\right) \neq 0
$$

by Proposition 2.2. Consequently, $f_{\mu}$ is nonzero.

Up to a nonzero multiplicative constant, $f_{\mu}$ is the unique elementary vector valued spherical function of degree $\mu$. It thus suffices to prove the induction step for $f_{\mu}$. By the construction of the vector valued spherical function $f$, we have

$$
\left.f\right|_{T}=\left(\left.g_{\mu}\right|_{T}\right)\left(\left.f_{0}\right|_{T}\right)
$$

in $\mathbb{C}\left[u^{ \pm 1}\right]$. Substituting the expansions (4.6) and (4.7) of $\left.g_{\mu}\right|_{T}$ and $f$, respectively, in (4.9), gives

$$
\left.\sum_{\substack{\nu \in \Lambda_{n}^{+} \\ \nu \leqslant \mu}} d_{\nu} f_{0}\right|_{T} m_{\nu}=\left.f\right|_{T}=\left.f_{\mu}\right|_{T}+\left.\sum_{\substack{\nu^{\prime} \in \Lambda_{n}^{+} \\ \nu^{\prime}<\mu}} f_{\nu^{\prime}}\right|_{T} .
$$

By the induction hypothesis applied to the $\left.f_{\nu^{\prime}}\right|_{T}\left(\nu^{\prime}<\mu\right)$, we conclude that (4.5) is valid for some $c_{\nu} \in \mathbb{C}$ and that $c_{\mu}=d_{\mu} \neq 0$. This completes the proof of the induction step.

As an immediate corollary of Proposition 4.5, we obtain the generalized Chevalley restriction theorem (see Theorem 1.21(i)).

Corollary 4.6. Let $f_{0} \in F_{\vec{\kappa}}^{\sigma, \tau}(0)$ be an elementary vector valued spherical function of degree zero. The restriction map $\left.\right|_{T}$ induces a linear bijection $\left.\right|_{T}:\left.F_{\vec{\kappa}}^{\sigma, \tau} \rightarrow f_{0}\right|_{T} \mathbb{C}\left[u^{ \pm 1}\right]^{W}$.

\section{The ground state}

We define a ground state $f_{0}$ (with respect to $(\vec{\kappa}, \sigma, \tau)$ ) to be an elementary vector valued spherical function $f_{0} \in F_{\vec{k}}^{\sigma, \tau}(0)$ of degree zero. Note that a ground state $f_{0}$ is unique up to nonzero scalar multiples. In this section we compute the radial part $\left.f_{0}\right|_{T}$ of a ground state $f_{0}$ explicitly. In the first subsection we show that it suffices to compute the ground state for $\vec{\kappa}=(0,0, \kappa)$ and $\vec{\kappa}=\left(\kappa_{1}, \kappa_{2}, 0\right)$ separately. The second and third subsections are devoted to the explicit computation of the ground state for these two special cases.

\subsection{Splitting of the ground state}

For the computation of the radial part of the ground state it is sufficient to compute the radial part for the special cases $\kappa_{1}=\kappa_{2}=0$ and $\kappa=0$ in view of the following lemma.

Lemma 5.1. Let $f \in F_{\left(\kappa_{1}, \kappa_{2}, 0\right)}^{\sigma, \tau}(0)$ and $f^{\prime} \in F_{(0,0, \kappa)}^{\sigma+2 \kappa_{2}, \tau+2 \kappa_{1}}(0)$ be ground states with respect to $\left(\kappa_{1}, \kappa_{2}, 0, \sigma, \tau\right)$ and $\left(0,0, \kappa, \sigma+2 \kappa_{2}, \tau+2 \kappa_{1}\right)$, respectively. Then $\left.\left.f\right|_{T} f^{\prime}\right|_{T}=\left.f_{0}\right|_{T}$ in $\mathbb{C}\left[u^{ \pm 1}\right]$ with $f_{0} \in F_{\vec{\kappa}}^{\sigma, \tau}(0)$ a ground state with respect to $(\vec{\kappa}, \sigma, \tau)$.

Proof. We proceed as in the first part of the proof of Proposition 4.5. We identify $V\left(\kappa_{2}\right)_{\sigma}$ with its unique copy in $L_{\delta\left(0, \kappa_{1}\right)^{\natural}}^{\sigma}$ and $V(0)_{\sigma+2 \kappa_{2}}$ with its unique copy in $L_{\delta(\kappa, 0)^{\natural}}^{\sigma+2 \kappa_{2}}$, and we fix nonzero vectors $u \in V\left(\kappa_{2}\right)_{\sigma}$ and $v \in V(0)_{\sigma+2 \kappa_{2}}$. We, furthermore, fix nonzero intertwiners

$$
\varphi \in \operatorname{Hom}_{\mathcal{A}_{\tau}}\left(L_{\delta\left(0, \kappa_{1}\right)^{\natural}}^{\tau}, V\left(\kappa_{1}\right)_{\tau}\right), \quad \phi \in \operatorname{Hom}_{\mathcal{A}_{\tau+2 \kappa_{1}}}\left(L_{\delta(\kappa, 0)^{\natural}}^{\tau+2 \kappa_{1}}, V(\kappa, 0)_{\tau+2 \kappa_{1}}\right),
$$

then the formulas

$$
f(X)=\varphi(X u), \quad f^{\prime}(X)=\phi(X v)
$$

for $X \in U_{q}(\mathfrak{g})$ define ground states $f \in F_{\left(\kappa_{1}, \kappa_{2}, 0\right)}^{\sigma, \tau}(0)$ and $f^{\prime} \in F_{(0,0, \kappa)}^{\sigma+2 \kappa_{2}, \tau+2 \kappa_{1}}(0)$. We now consider the function $f_{0}(X)=\psi(X(u \otimes v))$ for $X \in U_{q}(\mathfrak{g})$, with

$$
\psi: L_{\delta\left(0, \kappa_{1}\right)^{\natural}} \otimes L_{\delta(\kappa, 0)^{\natural}} \rightarrow V\left(\kappa_{1}\right)_{\tau} \otimes V(\kappa, 0)_{\tau+2 \kappa_{1}} \simeq V\left(\kappa, \kappa_{1}\right)_{\tau}
$$




\section{SPHERICAL FUnCTIONS AND MACDONALD-KOORNWINDER POLYNOMIALS}

defined by $\psi\left(w \otimes w^{\prime}\right)=\varphi(w) \otimes \phi\left(w^{\prime}\right)$ for $w \in L_{\delta\left(0, \kappa_{1}\right)^{\natural}}$ and $w^{\prime} \in L_{\delta(\kappa, 0)^{\natural}}$ (see Corollary 4.4 for the isomorphism $V\left(\kappa_{1}\right)_{\tau} \otimes V(\kappa, 0)_{\tau+2 \kappa_{1}} \simeq V\left(\kappa, \kappa_{1}\right)_{\tau}$ of $\mathcal{A}_{\tau}$-modules in (5.1)). Similarly as in the proof of Proposition 4.5 we have

$$
\left.f_{0}\right|_{T}=\left(\left.f\right|_{T}\right)\left(\left.f^{\prime}\right|_{T}\right)
$$

in $\mathbb{C}\left[u^{ \pm 1}\right]$ and $0 \neq f_{0} \in F_{\vec{\kappa}}^{\sigma, \tau}$. In the expansion of $f_{0}$ in elementary vector valued spherical functions, there is only a contribution of degree zero in view of (4.4) and Corollary 1.18; hence, we conclude that $f_{0} \in F_{\left(\kappa_{1}, \kappa_{2}, \kappa\right)}^{\sigma, \tau}(0)$ is the ground state with respect to $(\vec{\kappa}, \sigma, \tau)$.

\subsection{The ground state for $\kappa_{1}=\kappa_{2}=0$}

We compute the radial part $\left.f_{0}\right|_{T} \in \mathbb{C}\left[u^{ \pm 1}\right]$ of the ground state $f_{0} \in F_{(0,0, \kappa)}^{\sigma, \tau}(0)$ by relating $\left.f_{0}\right|_{T}$ to the ground state for vector valued $U_{q}(\mathfrak{g l}(n))$-characters, which in turn was computed explicitly by Etingof and Kirillov [EK94].

We use the notation of $\S 1$. Denote $\left(\rho_{\kappa}, V_{\kappa}\right)$ for the finite-dimensional, irreducible $U_{q}(\mathfrak{g l}(n))$ representation $L_{(n-1) \kappa,(-\kappa)^{n-1}}$. We view an element $f \in \mathbb{C}_{q}[\mathrm{GL}(n ; \mathbb{C})]^{\otimes 2} \otimes V$ as a linear map $f$ : $U_{q}(\mathfrak{g l}(n))^{\otimes 2} \rightarrow V$.

Definition 5.2. Let $\widetilde{F}_{\kappa}$ be the space of functions $f \in \mathbb{C}_{q}[\mathrm{GL}(n ; \mathbb{C})]^{\otimes 2} \otimes V_{\kappa}$ satisfying

$$
\begin{aligned}
& f\left(X \Delta^{\mathrm{op}}(a)\right)=\epsilon(a) f(X), \quad \forall a \in U_{q}(\mathfrak{g l}(n)), \\
& f\left(\Delta^{\mathrm{op}}(b) X\right)=\rho_{\kappa}(b) f(X), \quad \forall b \in U_{q}(\mathfrak{g l}(n))
\end{aligned}
$$

for all $X \in U_{q}(\mathfrak{g l}(n))^{\otimes 2}$.

Recall from Lemma 1.14 that the isomorphism $U_{q}(\mathfrak{g l}(n)) \simeq \mathcal{E}$ of Lemma 1.8 implies that the restriction of $V\left(\kappa, \kappa_{1}\right)_{\tau}$ to $\mathcal{E}$ is isomorphic to $V_{\kappa}$ as $U_{q}(\mathfrak{g r}(n))$-modules. In the following lemma we, furthermore, use the natural identification $U_{q}(\mathfrak{g l}(n))^{\otimes 2} \simeq U_{q}(\mathfrak{k}) \subset U_{q}(\mathfrak{g})$, cf. $\S 1.3$.

LEMma 5.3. The assignment

$$
f \mapsto f \circ(\operatorname{Id} \otimes \psi),
$$

with $\psi$ defined by (1.14), defines a linear map $F_{\vec{\kappa}}^{\sigma, \tau} \rightarrow \widetilde{F}_{\kappa}$.

Proof. With the identifications discussed above, the lemma follows immediately from the explicit form of the isomorphism $U_{q}(\mathfrak{g l}(n)) \simeq \mathcal{E}$, see Lemma 1.8.

For a left $U_{q}(\mathfrak{g l}(n))$-module $V$, we consider its linear dual $V^{*}$ as a $U_{q}(\mathfrak{g l}(n))$-module by

$$
(X \phi)(v)=\phi\left(S^{-1}(X) v\right), \quad X \in U_{q}(\mathfrak{g l}(n)), \phi \in V^{*}, v \in V .
$$

Let $\left(V \otimes V^{*}\right)^{\text {op }}$ be the $U_{q}(\mathfrak{g l}(n))$-module with representation space $V \otimes V^{*}$ and $U_{q}(\mathfrak{g l}(n))$-action

$$
X(v \otimes \phi)=\sum X_{2} v \otimes X_{1} \phi, \quad X \in U_{q}(\mathfrak{g l}(n)), v \in V, \phi \in V^{*} .
$$

Lemma 5.4. Let $V$ be a finite-dimensional $U_{q}(\mathfrak{g l}(n))$-module. The $U_{q}(\mathfrak{g l}(n))$-intertwiners $\xi:(V \otimes$ $\left.V^{*}\right)^{\text {op }} \rightarrow V_{\kappa}$ are in bijective correspondence with $U_{q}(\mathfrak{g l}(n))$-intertwiners $\xi^{*}: V \rightarrow V \otimes V_{\kappa}$, where $V \otimes V_{\kappa}$ is now considered as a $U_{q}(\mathfrak{g l}(n))$-module by

$$
X(v \otimes w)=\sum X_{1} v \otimes X_{2} w, \quad X \in U_{q}(\mathfrak{g l}(n)), v \in V, w \in V_{\kappa} .
$$

The bijective correspondence is explicitly given by the formula

$$
(\phi \otimes \operatorname{Id}) \xi^{*}(v)=\xi\left(v \otimes K^{2 \delta_{n}} \phi\right), \quad \phi \in V^{*}, v \in V
$$

where (recall) $K^{2 \delta_{n}}=K_{1}^{2(n-1)} K_{2}^{2(n-2)} \cdots K_{n-1}^{2} \in U_{q}(\mathfrak{g l}(n))$.

Proof. The standard proof is left to the reader. 


\section{A. A. Oblomkov and J. V. Stokman}

Let $V$ be a finite-dimensional $U_{q}(\mathfrak{g l}(n))$-module with basis $\left\{v_{i}\right\}$ and corresponding dual basis $\left\{v_{i}^{*}\right\}$. Let $\xi:\left(V \otimes V^{*}\right)^{\text {op }} \rightarrow V_{\kappa}$ be an $U_{q}(\mathfrak{g l}(n))$-intertwiner. Then

$$
g_{V, \xi}(\cdot)=\sum_{i} \xi\left(\cdot\left(v_{i} \otimes v_{i}^{*}\right)\right)
$$

defines an element in $\widetilde{F}_{\kappa}$. Any element in $\widetilde{F}_{\kappa}$ is of this form for some $V$ and $\xi$.

Lemma 5.5. The radial part $\left.f\right|_{T} \in \mathbb{C}\left[u^{ \pm 1}\right]$ of a vector valued spherical function $f \in F_{\vec{\kappa}}^{\sigma, \tau}$ is divisible by

$$
I=u^{\delta(\kappa, 0)} \prod_{1 \leqslant i<j \leqslant n}\left(q^{2} u_{i}^{-1} u_{j} ; q^{2}\right)_{\kappa}
$$

in $\mathbb{C}\left[u^{ \pm 1}\right]$. Moreover, the quotient $\left.f\right|_{T} / I \in \mathbb{C}\left[u^{ \pm 1}\right]$ is $S_{n}$-invariant.

Proof. Let $f \in F_{\vec{\kappa}}^{\sigma, \tau}$ be a vector valued spherical function and set $g=f \circ(\operatorname{Id} \otimes \psi) \in \widetilde{F}_{\kappa}$. Then $g=g_{V, \xi}$ for some finite-dimensional $U_{q}(\mathfrak{g l}(n))$-module $V$ and some $U_{q}(\mathfrak{g l}(n))$-intertwiner $\xi:\left(V \otimes V^{*}\right)^{\mathrm{op}} \rightarrow V_{\kappa}$. Let $\lambda \in P_{2 n}$, then

$$
\left.f\right|_{T}\left(q^{\lambda}\right)=f\left(K^{\lambda-\delta_{2 n}}\right)=g\left(K^{\lambda^{\ddagger}-\left(1^{n}\right)} \otimes K^{2 \delta_{n}}\right)
$$

with

$$
\lambda^{\ddagger}=\left(\lambda_{1}-\lambda_{2 n}, \lambda_{2}-\lambda_{2 n-1}, \ldots, \lambda_{n}-\lambda_{n+1}\right) \in P_{n} .
$$

By the previous lemma we thus obtain

$$
\begin{aligned}
\left.f\right|_{T}\left(q^{\lambda}\right) & =\sum_{i} \xi\left(K^{\lambda^{\ddagger}-\left(1^{n}\right)} v_{i} \otimes K^{2 \delta_{n}} v_{i}^{*}\right) \\
& =\sum_{i}\left(v_{i}^{*} \otimes \operatorname{Id}\right) \xi^{*}\left(K^{\lambda^{\ddagger}-\left(1^{n}\right)} v_{i}\right) \\
& =\left.\operatorname{Tr}\right|_{V}\left(\xi^{*}\left(K^{\lambda^{\ddagger}-\left(1^{n}\right)}(\cdot)\right)\right) .
\end{aligned}
$$

The results of Etingof and Kirillov [EK94] imply

$$
\left.\operatorname{Tr}\right|_{V}\left(\xi^{*}\left(K^{\mu}(\cdot)\right)\right)=I\left(q^{\mu}\right) p\left(q^{\mu}\right), \quad \forall \mu \in P_{n}
$$

with $q^{\mu}=\left(q^{\mu_{1}}, q^{\mu_{2}}, \ldots, q^{\mu_{n}}\right)$ and $p \in \mathbb{C}\left[u^{ \pm 1}\right]^{S_{n}}$. Since $I \in \mathbb{C}\left[u^{ \pm 1}\right]$ is a homogeneous Laurent polynomial, we arrive at the desired result.

We continue our analysis of the ground state for $\kappa_{1}=\kappa_{2}=0$ following the method of Kirillov [Kir95]. We express the elements $B_{j}(j \neq n)$ and $B_{n}^{\sigma}$ of $\mathcal{A}_{\sigma}$ by

$$
B_{j}=\widetilde{y}_{j}+\widetilde{x}_{2 n-j}, \quad B_{n}^{\sigma}=\widetilde{y}_{n}+\widetilde{x}_{n}+\vartheta_{0}\left(q^{\sigma}\right) K_{n}^{-2}
$$

with

and with $\vartheta_{l}$ given by

$$
\widetilde{x}_{j}=K_{2 n-j}^{-1} x_{j} K_{j}^{-1}, \quad \widetilde{y}_{j}=y_{j} K_{j+1}^{-1} K_{2 n-j}^{-1},
$$

$$
\vartheta_{l}(s)=\frac{s^{-1}-s q^{-2 l}}{q-q^{-1}}, \quad l \in \mathbb{Z} ;
$$

cf. Lemma 4.3. We define elements $E_{n-1}, E_{n}, F_{n-1}, F_{n} \in U_{q}(\mathfrak{g})$ by

$$
\begin{array}{ll}
E_{n-1}=\widetilde{x}_{n+1}, & E_{n}=\widetilde{x}_{n} \widetilde{x}_{n+1}-\widetilde{x}_{n+1} \widetilde{x}_{n}, \\
F_{n-1}=\widetilde{y}_{n-1}, & F_{n}=\widetilde{y}_{n} \widetilde{y}_{n-1}-q^{-2} \widetilde{y}_{n-1} \widetilde{y}_{n} .
\end{array}
$$

Direct computations lead to the following lemma. 


\section{SPHERICAL FUnCTIONS AND MACDONALD-KOORNWINDER POLYNOMIALS}

LEMMA 5.6.

(i) For any $\sigma$,

$$
\begin{aligned}
& E_{n}=B_{n}^{\sigma} E_{n-1}-E_{n-1} B_{n}^{\sigma}, \\
& F_{n}=B_{n}^{\sigma} F_{n-1}-q^{-2} F_{n-1} B_{n}^{\sigma} .
\end{aligned}
$$

(ii) The following commutation relations hold in $U_{q}(\mathfrak{g})$,

$$
\begin{gathered}
E_{n-1} F_{n-1}=q^{2} F_{n-1} E_{n-1}, \quad E_{n-1} F_{n}=q^{2} F_{n} E_{n-1}, \\
E_{n} F_{n-1}=F_{n-1} E_{n}, \quad E_{n} F_{n}=q^{2} F_{n} E_{n}+\left(q^{-2}-1\right) F_{n-1} E_{n-1} C_{n}^{-2} .
\end{gathered}
$$

For an ordered $r$-tuple $J=\left(j_{1}, j_{2}, \ldots, j_{r}\right)$ with $j_{s} \in\{n-1, n\}$ and $r \leqslant \kappa$ we write

$$
F_{J}=F_{j_{1}} F_{j_{2}} \cdots F_{j_{r}}, \quad E_{J}=E_{j_{r}} \cdots E_{j_{2}} E_{j_{1}}
$$

and

$$
r_{J, n-1}=\#\left\{s \mid j_{s}=n-1\right\}, \quad r_{J, n}=r-r_{J, n-1} .
$$

We, furthermore, define the Laurent polynomial $\Delta_{J} \in \mathbb{C}\left[u^{ \pm 1}\right] \subset \mathbb{C}\left[z^{ \pm 1}\right]$ by

$$
\Delta_{J}=\prod_{s, t}\left(1-q^{2 s^{2}+2 s t+2 t^{2}}\left(u_{n-1}^{-1} u_{n}\right)^{s}\left(u_{n-1}^{-1} u_{n}^{-1}\right)^{t}\right),
$$

where the product is taken over $(s, t) \in \mathbb{Z}_{\geqslant 0}^{\times 2}$ with $0<s+t \leqslant r$ and $t \leqslant r_{J, n}$. For $J=\emptyset$, we write $F_{\emptyset}=E_{\emptyset}=1, r_{\emptyset, n-1}=r_{\emptyset, n}=0$ and $\Delta_{\emptyset}=1$. Using the notation of Lemma 1.13, we can now formulate the following result.

Lemma 5.7. Let $f \in F_{(0,0, \kappa)}^{\sigma, \tau}$ be a vector valued spherical function. Let $r \in \mathbb{Z}_{\geqslant 0}$ with $r \leqslant \kappa$ and fix an ordered $r$-tuple $J$ with coefficients from $\{n-1, n\}$. Then there exists a Laurent polynomial $P_{J} \in \mathbb{C}\left[z^{ \pm 1}\right]$ such that

$$
\Delta_{J}\left(q^{\lambda}\right) f\left(F_{J} K^{\lambda-\delta}\right)=\left.P_{J}\left(q^{\lambda}\right) f\right|_{T}\left(q^{\lambda}\right) r_{\left(\kappa^{n-2}, \kappa-r, \kappa+r\right)}
$$

for all $\lambda \in P_{2 n}$.

Proof. First note that the four elements $Y=E_{n-1}, E_{n}, F_{n-1}, F_{n}$ all have the same restricted weight,

$$
C_{j} Y=q^{\delta_{j, n}-\delta_{j, n-1}} Y C_{j}, \quad \forall j=1, \ldots, n .
$$

In particular, we conclude that

$$
f\left(F_{J} K^{\lambda-\delta}\right) \in \operatorname{span}\left\{r_{\left(\kappa^{n-2}, \kappa-r, \kappa+r\right)}\right\}
$$

cf. Lemma 1.13, hence

$$
f\left(F_{J} K^{\lambda-\delta}\right)=f_{J}\left(q^{\lambda}\right) r_{\left(\kappa^{n-2}, \kappa-r, \kappa+r\right)}, \quad \forall \lambda \in P_{2 n}
$$

for a unique Laurent polynomial $f_{J} \in \mathbb{C}\left[z^{ \pm 1}\right]$. Furthermore, we may and will view all formulas below as identities in $\mathbb{C}\left[z^{ \pm 1}\right]$ by identifying $\mathbb{C} r_{\left(\kappa^{n-2}, \kappa-r, \kappa+r\right)} \simeq \mathbb{C}$.

We thus need to prove the existence of a Laurent polynomial $P_{J} \in \mathbb{C}\left[z^{ \pm 1}\right]$ such that $\Delta_{J} f_{J}=$ $\left.P_{J} f\right|_{T}$. To avoid unnecessary technicalities we avoid the use of the underlying root data as much as possible. Let $\mathcal{F}_{r}$ be the finite set of $r$-tuples $J=\left(j_{1}, j_{2}, \ldots, j_{r}\right)$ with $j_{s} \in\{n-1, n\}$, and write $\mathcal{F}^{\kappa}=\bigcup_{0 \leqslant r \leqslant \kappa} \mathcal{F}_{r}$. For $I, J \in \mathcal{F}^{\kappa}$ we write $I<J$ if $\# I \lesseqgtr \# J$ and $r_{I, n} \leqslant r_{J, n}$, or if $\# I=\# J$ and $r_{I, n} \lesseqgtr r_{J, n}$. We prove the lemma by induction to $J \in \mathcal{F}^{\kappa}$ along $<$. For the smallest element $J=\emptyset \in \mathcal{F}_{0}$, we have $\Delta_{\emptyset}=1, f_{\emptyset}=\left.f\right|_{T}$, hence we can take $P_{\emptyset}=1$. Suppose there exists a Laurent polynomial $P_{J}$ such that $\Delta_{J} f_{J}=\left.P_{J} f\right|_{T}$ for all $J \in \mathcal{F}^{\kappa}$ with $J<I=\left(i_{1}, i_{2}, \ldots, i_{r}\right) \in \mathcal{F}_{r}$. For simplicity, we write $r_{1}=r_{I, n-1}$ and $r_{2}=r_{I, n}$. 


\section{A. A. Oblomkov and J. V. Stokman}

We write $g \equiv g^{\prime}$ for $g, g^{\prime} \in \mathbb{C}\left[z^{ \pm 1}\right]$ if $g-g^{\prime}$ lies in the ideal of $\mathbb{C}\left[z^{ \pm 1}\right]$ generated by the $f_{J}$ for $J \in \mathcal{F}^{\kappa}$ with $J<I$. Sometimes it is convenient to write $g\left(q^{\lambda}\right) \equiv g^{\prime}\left(q^{\lambda}\right)$, where we have formally evaluated the equivalence $g \equiv g^{\prime}$ of the Laurent polynomials $g$ and $g^{\prime}$ in $z=q^{\lambda}$ for arbitrary $\lambda \in P_{2 n}$.

To prove the existence of the Laurent polynomial $P_{I}$ satisfying $\Delta_{I} f_{I}=\left.P_{I} f\right|_{T}$, it suffices to show that

$$
\left(1-q^{2 r_{1}^{2}+2 r_{1} r_{2}+2 r_{2}^{2}}\left(u_{n-1}^{-1} u_{n}\right)^{r_{1}}\left(u_{n-1}^{-1} u_{n}^{-1}\right)^{r_{2}}\right) f_{I} \equiv 0
$$

due to the induction hypothesis and the fact that $\Delta_{J}$ divides $\Delta_{I}$ in $\mathbb{C}\left[u^{ \pm 1}\right] \subset \mathbb{C}\left[z^{ \pm 1}\right]$ when $J<I$. We start by noting that

$$
\begin{aligned}
f\left(F_{n-1} X\right) & =-f\left(E_{n-1} X\right)+\rho(\kappa, 0)_{\tau}\left(B_{n-1}\right)(f(X)), \\
f\left(F_{n} X\right) & =-q^{-2} f\left(E_{n} X\right)+\left(1-q^{-2}\right) \rho(\kappa, 0)_{\tau}\left(B_{n}^{\tau}\right)\left(f\left(F_{n-1} X\right)\right)+\rho(\kappa, 0)_{\tau}\left(\left[B_{n}^{\tau}, B_{n-1}\right]\right)(f(X))
\end{aligned}
$$

and

$$
\begin{aligned}
f\left(X E_{n-1}\right) & =-f\left(X F_{n-1}\right), \\
f\left(X E_{n}\right) & =-f\left(X F_{n}\right)+\left(1-q^{-2}\right) \vartheta_{0}\left(q^{\sigma}\right) f\left(X F_{n-1}\right)
\end{aligned}
$$

for any $X \in U_{q}(\mathfrak{g})$, which follows easily from Lemma 5.6(i) and the fact that $f$ is a vector valued spherical function. Denote $I^{\prime}=\left(i_{2}, i_{3}, \ldots, i_{r}\right)$, which is $<I$. Then it follows from (5.5) that

$$
f_{I}\left(q^{\lambda}\right) \equiv-q^{-2 \delta_{i_{1}, n}} f\left(E_{i_{1}} F_{I^{\prime}} K^{\lambda-\delta}\right) .
$$

By Lemma 5.6(ii) we now conclude that

$$
f_{I}\left(q^{\lambda}\right) \equiv \begin{cases}-q^{2(r-1)} f\left(F_{I^{\prime}} E_{i_{1}} K^{\lambda-\delta}\right), & \text { if } i_{1}=n-1, \\ -q^{-2} q^{2\left(r_{2}-1\right)} f\left(F_{I^{\prime}} E_{i_{1}} K^{\lambda-\delta}\right), & \text { if } i_{1}=n .\end{cases}
$$

Continuing inductively while taking (5.6) into account, we obtain

$$
\begin{aligned}
f_{I}\left(q^{\lambda}\right) & \equiv(-1)^{r} q^{-2 r_{2}+r_{2}\left(r_{2}-1\right)+2 \sum_{s: i_{s}=n-1}(r-s)} f\left(E_{I} K^{\lambda-\delta}\right) \\
& \equiv(-1)^{r} q^{r_{1}+r_{2}\left(r_{2}-1\right)+2 \sum_{s: i_{s}=n-1}(r-s)} q^{-r_{2} \lambda_{n}-r_{1} \lambda_{n+1}+r \lambda_{n+2}} f\left(K^{\lambda-\delta} E_{I}\right) .
\end{aligned}
$$

We follow a similar procedure to return from $f\left(K^{\lambda-\delta} E_{I}\right)$ to $f_{I}\left(q^{\lambda}\right)$. The starting point is

$$
f\left(K^{\lambda-\delta} E_{I}\right) \equiv-f\left(K^{\lambda-\delta} E_{I^{\prime}} F_{i_{1}}\right),
$$

which follows from (5.6). Moving $F_{i_{1}}$ to the left using Lemma 5.6(ii) gives

$$
f\left(K^{\lambda-\delta} E_{I}\right) \equiv \begin{cases}-q^{2\left(r_{1}-1\right)} f\left(K^{\lambda-\delta} F_{i_{1}} E_{I^{\prime}}\right), & \text { if } i_{1}=n-1, \\ -q^{2(r-1)} f\left(K^{\lambda-\delta} F_{i_{1}} E_{I^{\prime}}\right), & \text { if } i_{1}=n .\end{cases}
$$

Repeating this procedure inductively gives

$$
\begin{aligned}
f\left(K^{\lambda-\delta} E_{I}\right) & \equiv(-1)^{r} q^{r_{1}\left(r_{1}-1\right)+2 \sum_{s: i_{s}=n}(r-s)} f\left(K^{\lambda-\delta} F_{I}\right) \\
& \equiv(-1)^{r} q^{r_{1}^{2}+2 r_{2}+2 \sum_{s: i_{s}=n}(r-s)} q^{-r \lambda_{n-1}+r_{1} \lambda_{n}+r_{2} \lambda_{n+1}} f_{I}\left(q^{\lambda}\right) .
\end{aligned}
$$

Combining (5.7) and (5.8) yields the induction step (5.4).

Lemma 5.8. The radial part $\left.f\right|_{T} \in \mathbb{C}\left[u^{ \pm 1}\right]$ of a vector valued spherical function $f \in F_{(0,0, \kappa)}^{\sigma, \tau}$ is divisible by $\left(1-q^{2 r} u_{n-1}^{-1} u_{n}^{-1}\right)$ for $r=1, \ldots, \kappa$.

Proof. It is easy to check that $\Delta_{\left(n^{\kappa}\right)}$ is divisible by $1-q^{2 r} u_{n-1}^{-1} u_{n}^{-1}$ in $\mathbb{C}\left[u^{ \pm 1}\right]$ for $r=1, \ldots, \kappa$. By the previous lemma it thus suffices to show that $P_{\left(n^{\kappa}\right)}$ is nonzero and relative prime to $1-q^{2 r} u_{n-1}^{-1} u_{n}^{-1}$ for $r=1, \ldots, \kappa$. Clearly it is enough to prove this in the classical limit $q=1$. We thus consider $q=e^{h}$ as a formal parameter and we repeat part of the computations of the proof of Lemma 5.7 for $J=\left(n^{r}\right)$, now modding out to $h \mathbb{C}[[h]]\left[z^{ \pm 1}\right]$. 


\section{Spherical FUnCTIONS AND MACDONALD-KOORNWINDER POLYNOMIALS}

We simplify the notation by writing

$$
\begin{aligned}
f\left(F_{n}^{s} K^{\lambda-\delta}\right) & =f_{s}\left(q^{\lambda}\right) r_{\left(\kappa^{n-2}, \kappa-s, \kappa+s\right)}, \\
f\left(E_{n} F_{n}^{s-1} K^{\lambda-\delta}\right) & =g_{s}\left(q^{\lambda}\right) r_{\left(\kappa^{n-2}, \kappa-s, \kappa+s\right)}
\end{aligned}
$$

for all $\lambda \in P_{2 n}$ and $s \in\{1, \ldots, \kappa\}$, with $f_{s}, g_{s} \in \mathbb{C}[[h]]\left[z^{ \pm 1}\right]$. Lemma 1.13 gives

$$
\rho(\kappa, 0)_{\tau}\left(\left[B_{n}^{\tau}, B_{n-1}\right]\right) f\left(F_{n}^{r-1} K^{\lambda-\delta}\right)=q^{2-\tau-3 r}[r-1-\kappa]_{q} f_{r-1}\left(q^{\lambda}\right) r_{\left(\kappa^{n-2}, \kappa-r, \kappa+r\right)},
$$

with

$$
[\alpha]_{q}=\frac{q^{\alpha}-q^{-\alpha}}{q-q^{-1}}
$$

the (symmetric) $q$-number. Identifying $\mathbb{C} r_{\left(\kappa^{n-2}, \kappa-r, \kappa+r\right)} \simeq \mathbb{C}$ as in the proof of Lemma 5.7, we obtain from the second formula of (5.5),

$$
f_{r}=-q^{-2} g_{r-1}+q^{2-\tau-3 r}[r-1-\kappa]_{q} f_{r-1} \bmod h .
$$

By Lemma 5.6(ii), we can move $E_{n}$ to the other side in the expression

$$
g_{r-1}\left(q^{\lambda}\right)=f\left(E_{n} F_{n}^{r-1} K^{\lambda-\delta}\right),
$$

after which we can use the second formula in (5.6) to replace $E_{n}$ by $-F_{n}$ modulo $h$. This yields

$$
g_{r-1}=-q^{2(r+1)} u_{n-1}^{-1} u_{n}^{-1} f_{r} \bmod h .
$$

Consequently,

$$
\left(1-q^{2 r} u_{n-1}^{-1} u_{n}^{-1}\right) f_{r}=q^{2-\tau-3 r}[r-1-\kappa]_{q} f_{r-1} \quad \bmod h,
$$

or, more precisely,

$$
\left(1-u_{n-1}^{-1} u_{n}^{-1}\right) f_{r}=(r-1-\kappa) f_{r-1} \bmod h
$$

for $r=1, \ldots, \kappa$. Thus $P_{\left(n^{\kappa}\right)}$ is nonzero, and

$$
P_{\left(n^{\kappa}\right)}=C\left(1-u_{n-1}^{-1} u_{n}^{-1}\right)^{-\kappa} \Delta_{\left(n^{\kappa}\right)} \bmod h,
$$

for some nonzero constant $C \in \mathbb{C}$. Using the explicit expression of $\Delta_{\left(n^{\kappa}\right)}$, it is easy to check that the right-hand side of $(5.9)(\bmod h)$ is relative prime to $1-u_{n-1}^{-1} u_{n}^{-1}$ in $\mathbb{C}\left[z^{ \pm 1}\right]$, which completes the proof of the lemma.

We are now in a position to determine the explicit form of the radial part of the ground state when $\kappa_{1}=\kappa_{2}=0$.

COROLlary 5.9. Let $f \in F_{(0,0, \kappa)}^{\sigma, \tau}(0)$ be a ground state. With a suitable normalization of $f$, we have

$$
\left.f\right|_{T}=u^{\delta(\kappa, 0)} \prod_{1 \leqslant i<j \leqslant n}\left(q^{2} u_{i}^{-1} u_{j}, q^{2} u_{i}^{-1} u_{j}^{-1} ; q^{2}\right)_{\kappa}
$$

in $\mathbb{C}\left[u^{ \pm 1}\right]$.

Proof. We write $\mathcal{I} \in \mathbb{C}\left[u^{ \pm 1}\right]$ for the right-hand side of (5.10). Lemma 5.5 and Lemma 5.8 show that $\left.f\right|_{T}$ is divisible by $\mathcal{I}$ in $\mathbb{C}\left[u^{ \pm 1}\right]$, so $\left.f\right|_{T}=\mathcal{I} p$ for some Laurent polynomial $p \in \mathbb{C}\left[u^{ \pm 1}\right]$. It remains to show that $p$ is a nonzero constant. For this we expand both sides of the equality $\left.f\right|_{T}=\mathcal{I} p$ in monomials $u^{\mu}\left(\mu \in \Lambda_{n}\right)$.

We write

$$
p=\sum_{\nu} e_{\mu} u^{\nu}
$$




\section{A. A. Oblomkov and J. V. Stokman}

and we denote $\nu_{+} \in \Lambda_{n}$ (respectively $\nu_{-} \in \Lambda_{n}$ ) for a maximal (respectively minimal) element with respect to the dominance order $\leqslant$ such that $e_{\nu_{+}} \neq 0 \neq e_{\nu_{-}}$. Observe that

$$
\mathcal{I}=\sum_{-\delta(\kappa, 0) \leqslant \nu \leqslant \delta(\kappa, 0)} c_{\nu} u^{\nu}
$$

with $c_{\nu} \in \mathbb{C}$ and $c_{-\delta(\kappa, 0)} \neq 0 \neq c_{\delta(\kappa, 0)}$. Consequently, in the expansion of $\mathcal{I} p$ in monomials $u^{\mu}$ $\left(\mu \in \Lambda_{n}\right)$, the coefficients of $u^{\delta(\kappa, 0)+\nu_{+}}$and $u^{-\delta(\kappa, 0)-\nu_{-}}$are nonzero.

On the other hand, Lemma 4.1 implies that

$$
\left.f\right|_{T}=\sum_{-\delta(\kappa, 0) \leqslant \nu \leqslant \delta(\kappa, 0)} d_{\nu} u^{\nu}
$$

with $d_{\nu} \in \mathbb{C}$ and $d_{-\delta(\kappa, 0)} \neq 0 \neq d_{\delta(\kappa, 0)}$. Hence the equality $\left.f\right|_{T}=\mathcal{I} p$ forces $\nu_{+} \leqslant 0$ and $\nu_{-} \geqslant 0$. This in turn implies $\nu_{+}=\nu_{-}=0$, and hence $p$ is a nonzero constant.

\subsection{The ground state for $\kappa=0$}

We start with the following preliminary lemma, which will be convenient for several computations in this subsection. Recall the definition of $\vartheta_{k}(s)$, see $(5.3)$.

Lemma 5.10. Let $W_{1}$ and $W_{2}$ be left $U_{q}(\mathfrak{g})$-modules and let $k \in \mathbb{Z}$.

(i) If $w_{0} \in W_{1}$ is a vector satisfying $B_{n}^{\sigma} w_{0}=\vartheta_{0}\left(q^{\sigma+k}\right) w_{0}$ and $C_{n} w_{0}=w_{0}$, then

$$
B_{n}^{\sigma}\left(w_{0} \otimes w^{\prime}\right)=w_{0} \otimes B_{n}^{\sigma+k} w^{\prime}, \quad \forall w^{\prime} \in W_{2}
$$

in the $U_{q}(\mathfrak{g})$-module $W_{1} \otimes W_{2}$.

(ii) If $w_{ \pm} \in W_{1}$ is a vector satisfying $B_{n}^{\sigma} w_{ \pm}=\vartheta_{k}\left( \pm q^{ \pm \sigma}\right) w_{ \pm}$and $C_{n} w_{ \pm}=q^{k} w_{ \pm}$, then

$$
B_{n}^{\sigma}\left(w_{ \pm} \otimes w^{\prime}\right)=w_{ \pm} \otimes q^{-k} B_{n}^{\sigma \mp k} w^{\prime}, \quad \forall w^{\prime} \in W_{2}
$$

in the $U_{q}(\mathfrak{g})$-module $W_{1} \otimes W_{2}$.

Proof. Recall that the generator $B_{n}^{\sigma} \in \mathcal{A}_{\sigma}$ is explicitly given by

$$
B_{n}^{\sigma}=y_{n} K_{n+1}^{-1} K_{n}^{-1}+K_{n}^{-1} x_{n} K_{n}^{-1}+\vartheta_{0}\left(q^{\sigma}\right) K_{n}^{-2} .
$$

The proof of (i) now follows immediately from the explicit formula for $\Delta\left(B_{n}^{\sigma}\right)$ (see Proposition 4.2) and from the fact that $C_{n}=C_{n+1}$. For the proof of (ii), let $w_{ \pm} \in W_{1}$ be as indicated in the lemma, and let $w^{\prime} \in W_{2}$. Then Proposition 4.2 implies

$$
\begin{aligned}
B_{n}^{\sigma}\left(w_{ \pm} \otimes w^{\prime}\right) & =w_{ \pm} \otimes q^{-k}\left(y_{n} K_{n+1}^{-1} K_{n}^{-1}+K_{n}^{-1} x_{n} K_{n}^{-1}+q^{k} \vartheta_{k}\left( \pm q^{ \pm \sigma}\right) K_{n}^{-2}\right) w^{\prime} \\
& =w_{ \pm} \otimes q^{-k} B_{n}^{\sigma \mp k} w^{\prime}
\end{aligned}
$$

since $q^{k} \vartheta_{k}\left( \pm q^{ \pm \sigma}\right)=\vartheta_{0}\left(q^{\sigma \mp k}\right)$.

In this subsection the realization of the fundamental, simple $U_{q}(\mathfrak{g})$-modules within the $q$-exterior algebra of the vector representation of $U_{q}(\mathfrak{g})$ is used to explicitly construct the one-dimensional $\mathcal{A}_{\sigma}$-modules $V\left(\kappa_{1}\right)_{\sigma}$ and $V\left(\kappa_{2}\right)_{\sigma}$ within simple $U_{q}(\mathfrak{g})$-modules. As a consequence, we obtain an explicit expression for the radial part of the ground state for $\kappa=0$.

The vector representation of $U_{q}(\mathfrak{g})$ is the $2 n$-dimensional vector space $V$ with linear basis $v_{i}$ $(i=1, \ldots, 2 n)$ and with $U_{q}(\mathfrak{g})$-action defined by

$$
K_{r}^{ \pm 1} v_{j}=q^{ \pm \delta_{r, j}} v_{j}, \quad x_{i} v_{j}=\delta_{i+1, j} v_{j-1}, \quad y_{i} v_{j}=\delta_{i, j} v_{j+1}
$$

for $r, j=1, \ldots, 2 n$ and $i=1, \ldots, 2 n-1$, with the convention that $v_{0}=v_{2 n+1}=0$. This is a realization of the finite-dimensional irreducible highest weight module $L_{\epsilon_{1}}$ of $U_{q}(\mathfrak{g})$, with highest 


\section{SPHERICAL FUnCTIONS AND MACDONALD-KOORNWINDER POLYNOMIALS}

weight vector $v_{1}$. Similarly, the dual vector representation is the $2 n$-dimensional vector space $V^{*}$ with basis $v_{i}^{*}(i=1, \ldots, 2 n)$ and with $U_{q}(\mathfrak{g})$-action given explicitly by

$$
K_{r}^{ \pm 1} v_{j}^{*}=q^{\mp \delta_{r, j}} v_{j}^{*}, \quad x_{i} v_{j}^{*}=-q^{-1} \delta_{i, j} v_{j+1}^{*}, \quad y_{i} v_{j}^{*}=-q \delta_{i+1, j} v_{j-1}^{*}
$$

for $r, j=1, \ldots, 2 n$ and $i=1, \ldots, 2 n-1$, with the convention that $v_{0}^{*}=v_{2 n+1}^{*}=0$ (it corresponds to the linear dual $V^{*}$ of $V$ viewed as $U_{q}(\mathfrak{g})$-module by $(X \phi)(v)=\phi(S(X) v)$ for $\phi \in V^{*}, v \in V$ and $\left.X \in U_{q}(\mathfrak{g})\right)$. This is a realization of the finite-dimensional irreducible highest weight module $L_{-\epsilon_{2 n}}$ of $U_{q}(\mathfrak{g})$, with highest weight vector $v_{2 n}^{*}$. For $s \in \mathbb{R} \backslash\{0\}$ we define $n$-dimensional subspaces $W(s)=\operatorname{span}\left\{w_{i}(s)\right\}_{i} \subseteq V$ and $W^{*}(s)=\operatorname{span}\left\{w_{i}^{*}(s)\right\}_{i} \subseteq V^{*}$ by

$$
w_{i}(s)=v_{i}+s^{-1} v_{2 n+1-i}, \quad w_{i}^{*}(s)=v_{i}^{*}+s^{-1} q^{2 i-2 n-1} v_{2 n+1-i}^{*}
$$

for $i=1, \ldots, n$.

Proposition 5.11. The vector spaces $W\left( \pm q^{ \pm \sigma}\right.$ ) (respectively $W^{*}\left( \pm q^{ \pm \sigma}\right)$ ) are inequivalent simple $\mathcal{A}_{\sigma}$-submodules of $V^{\sigma}$ (respectively $V^{* \sigma}$ ). The irreducible decompositions of the semisimple $\mathcal{A}_{\sigma^{-}}$ modules $V^{\sigma}$ and $V^{* \sigma}$ are

$$
V^{\sigma}=W\left(q^{\sigma}\right) \oplus W\left(-q^{-\sigma}\right), \quad V^{* \sigma}=W^{*}\left(q^{\sigma}\right) \oplus W^{*}\left(-q^{-\sigma}\right) .
$$

Proof. The action of the algebraic generators of $\mathcal{E} \subset \mathcal{A}_{\sigma}$ (see Lemma 1.8) on $w_{i}(s)$ and $w_{i}^{*}(s)$ is given by the formulas

$$
\begin{gathered}
C_{j} w_{i}(s)=q^{\delta_{i, j}} w_{i}(s), \quad C_{j} w_{i}^{*}(s)=q^{-\delta_{i, j}} w_{i}^{*}(s), \quad i, j=1, \ldots, n, \\
B_{i} w_{i}(s)=w_{i+1}(s), \quad B_{i} w_{i+1}^{*}(s)=-q^{2} w_{i}^{*}(s), \quad i=1, \ldots, n-1, \\
B_{2 n+1-i} w_{i}(s)=w_{i-1}(s), \quad B_{2 n+1-i} w_{i-1}^{*}(s)=-w_{i}^{*}(s), \quad i=2, \ldots, n, \\
B_{j} w_{i}(s)=0, \quad B_{j} w_{i}^{*}(s)=0, \quad \text { otherwise. }
\end{gathered}
$$

Hence $W(s)$ (respectively $W^{*}(s)$ ) is a $\mathcal{E}$-submodule of $V$ (respectively $V^{*}$ ) which is isomorphic to the vector representation (respectively dual vector representation) of $U_{q}(\mathfrak{g l}(n)) \simeq \mathcal{E}$, cf. Lemma 1.8. In particular, $W(s)$ and $W^{*}(s)$ are simple $\mathcal{E}$-modules.

Recall the notation (5.3). By a direct computation one can verify that

$$
B_{n}^{\sigma} w_{i}(s)=\vartheta_{0}\left(q^{\sigma}\right) w_{i}(s), \quad B_{n}^{\sigma} w_{i}^{*}(s)=\vartheta_{0}\left(q^{\sigma}\right) w_{i}^{*}(s)
$$

for all $i=1, \ldots, n-1$, and

$$
B_{n}^{\sigma} w_{n}\left( \pm q^{ \pm \sigma}\right)=\vartheta_{1}\left( \pm q^{ \pm \sigma}\right) w_{n}\left( \pm q^{ \pm \sigma}\right), \quad B_{n}^{\sigma} w_{n}^{*}\left( \pm q^{ \pm \sigma}\right)=\vartheta_{-1}\left( \pm q^{ \pm \sigma}\right) w_{n}^{*}\left( \pm q^{ \pm \sigma}\right) .
$$

It is now clear that $W\left( \pm q^{ \pm \sigma}\right) \subset V^{\sigma}$ are simple $\mathcal{A}_{\sigma^{-}}$submodules, and that $V^{\sigma}=W\left(q^{\sigma}\right) \oplus W\left(-q^{-\sigma}\right)$ (and similarly for $V^{* \sigma}$ ). To prove that $W\left(q^{\sigma}\right) \not 千 W\left(-q^{-\sigma}\right)$ (respectively $W^{*}\left(q^{\sigma}\right) \not W^{*}\left(-q^{-\sigma}\right)$ ) as $\mathcal{A}_{\sigma^{-}}$-modules, it suffices to observe that the spectrum of $B_{n}^{\sigma}$ is different. This follows from the fact that $\vartheta_{k}\left(q^{\sigma}\right)=\vartheta_{k}\left(-q^{-\sigma}\right)$ for $\sigma \in \mathbb{R}$ implies $k=0$.

We now consider the $q$-exterior algebras of $V$ and $V^{*}$, cf. [NYM93]. Let $T(V)$ and $T\left(V^{*}\right)$ be the tensor algebras of $V$ and $V^{*}$, respectively. Let $J \subset T(V)$ be the graded, two-sided ideal generated by the tensors $v_{i} \otimes v_{i}$ and $v_{r} \otimes v_{s}+q^{-1} v_{s} \otimes v_{r}$ for $i=1, \ldots, 2 n$ and $1 \leqslant r<s \leqslant 2 n$. Similarly, we write $J_{*} \subset T\left(V^{*}\right)$ for the graded, two-sided ideal generated by the tensors $v_{i}^{*} \otimes v_{i}^{*}$ and $v_{s}^{*} \otimes v_{r}^{*}+q^{-1} v_{r}^{*} \otimes v_{s}^{*}$ for $i=1, \ldots, 2 n$ and $1 \leqslant r<s \leqslant 2 n$. The exterior algebras $\Lambda(V)$ and $\Lambda\left(V^{*}\right)$ are defined to be the graded algebras $T(V) / J$ and $T\left(V^{*}\right) / J_{*}$, respectively. The action of $U_{q}(\mathfrak{g})$ on $V$ and $V^{*}$ naturally extends to a grading-preserving, left $U_{q}(\mathfrak{g})$-module algebra structure on the exterior algebras $\Lambda(V)$ and $\Lambda\left(V^{*}\right)$. We denote by $\wedge$ the products in $\Lambda(V)$ and $\Lambda\left(V^{*}\right)$. Let $\Lambda^{m}(V)$ and $\Lambda^{m}\left(V^{*}\right)$ be the $m$ th graded pieces of $\Lambda(V)$ and $\Lambda\left(V^{*}\right)$ respectively, then

$$
\Lambda(V)=\bigoplus_{m=0}^{2 n} \Lambda^{m}(V), \quad \Lambda\left(V^{*}\right)=\bigoplus_{m=0}^{2 n} \Lambda^{m}\left(V^{*}\right)
$$




\section{A. A. Oblomkov and J. V. Stokman}

and

$$
\Lambda^{m}(V) \simeq L_{\left(1^{m}, 0^{2 n-m}\right)}, \quad \Lambda^{m}\left(V^{*}\right) \simeq L_{\left(0^{2 n-m},-1^{m}\right)}
$$

as $U_{q}(\mathfrak{g})$-modules. For $I=\left\{i_{1}, \ldots, i_{m}\right\} \subseteq\{1, \ldots, 2 n\}$ with $i_{1}<i_{2}<\cdots<i_{m}$ we define

$$
v_{I}=v_{i_{1}} \wedge v_{i_{2}} \wedge \cdots \wedge v_{i_{m}}, \quad v_{I}^{*}=v_{i_{1}}^{*} \wedge v_{i_{2}}^{*} \wedge \cdots \wedge v_{i_{m}}^{*} .
$$

Then $\left\{v_{I} \mid \# I=m\right\}$ and $\left\{v_{I}^{*} \mid \# I=m\right\}$ are linear bases of $\Lambda^{m}(V)$ and $\Lambda^{m}\left(V^{*}\right)$, respectively. The action of $U_{q}(\mathfrak{g})$ on these basis elements is explicitly given by

$$
\begin{gathered}
K_{i}^{ \pm 1} v_{I}=q^{ \pm \delta(i, I)} v_{I}, \quad x_{i} v_{I}=v_{(I \backslash\{i+1\}) \cup\{i\}}, \quad y_{i} v_{I}=v_{(I \backslash\{i\}) \cup\{i+1\}}, \\
K_{i}^{ \pm 1} v_{I}^{*}=q^{\mp \delta(i, I)} v_{I}^{*}, \quad x_{i} v_{I}^{*}=-q^{-1} v_{(I \backslash\{i\}) \cup\{i+1\}}^{*}, \quad y_{i} v_{I}^{*}=-q v_{(I \backslash\{i+1\}) \cup\{i\}}^{*},
\end{gathered}
$$

where $\delta(i, I)=1$ if $i \in I$ and $\delta(i, I)=0$ otherwise, and where $v_{(I \backslash\{k\}) \cup\{l\}}=0$ and $v_{(I \backslash\{k\}) \cup\{l\}}^{*}=0$ if $k \notin I$ or if $l \in I$. Note, in particular, that $v_{\{1, \ldots, m\}} \in \Lambda^{m}(V)$ and $v_{\{2 n-m+1, \ldots, 2 n\}}^{*} \in \Lambda^{m}\left(V^{*}\right)$ are highest weight vectors. The formulas

$$
\left\langle v_{I}, v_{J}\right\rangle=\delta_{I, J}, \quad\left\langle v_{I}^{*}, v_{J}^{*}\right\rangle_{*}=q^{2 \sum_{i \in I} i} \delta_{I, J}
$$

define $*$-unitary scalar products on $\Lambda(V)$ and $\Lambda\left(V^{*}\right)$, respectively.

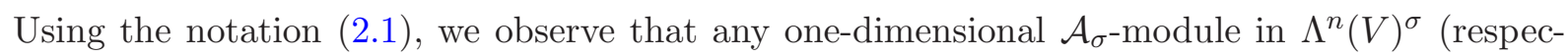
tively $\left.\Lambda^{n}\left(V^{*}\right)^{\sigma}\right)$ necessarily lies in the subspace $\Lambda^{n}(V)_{\left(1^{n}\right)}$ (respectively $\left.\Lambda^{n}\left(V^{*}\right)_{\left(-1^{n}\right)}\right)$. This follows easily using Lemma 1.8, since a one-dimensional $U_{q}(\mathfrak{g l}(n))$-module is of highest weight $\left(m^{n}\right) \in P_{n}^{+}$ for some integer $m$. For $\underline{s}=\left(s_{1}, \ldots, s_{n}\right)$ an $n$-tuple of nonzero reals, we define $\xi(\underline{s}) \in \Lambda^{n}(V)_{\left(1^{n}\right)}$ and $\xi^{*}(\underline{s}) \in \Lambda^{n}\left(V^{*}\right)_{\left(-1^{n}\right)}$ by

$$
\begin{aligned}
\xi(\underline{s}) & =w_{1}\left(s_{1}\right) \wedge w_{2}\left(s_{2}\right) \wedge \cdots \wedge w_{n}\left(s_{n}\right), \\
\xi^{*}(\underline{s}) & =w_{1}^{*}\left(s_{1}\right) \wedge w_{2}^{*}\left(s_{2}\right) \wedge \cdots \wedge w_{n}^{*}\left(s_{n}\right) .
\end{aligned}
$$

It is also convenient to use the opposite elements $\xi_{\text {op }}(\underline{s}) \in \Lambda^{n}(V)_{\left(1^{n}\right)}$ and $\xi_{\text {op }}^{*}(\underline{s}) \in \Lambda^{n}\left(V^{*}\right)_{\left(-1^{n}\right)}$, defined by

$$
\begin{aligned}
& \xi_{\mathrm{op}}(\underline{s})=w_{n}\left(s_{n}\right) \wedge \cdots \wedge w_{2}\left(s_{2}\right) \wedge w_{1}\left(s_{1}\right), \\
& \xi_{\mathrm{op}}^{*}(\underline{s})=w_{n}^{*}\left(s_{n}\right) \wedge \cdots \wedge w_{2}^{*}\left(s_{2}\right) \wedge w_{1}^{*}\left(s_{1}\right) .
\end{aligned}
$$

For $1 \leqslant i<j \leqslant n$ we have

$$
\begin{aligned}
w_{i}\left(s_{i}\right) \wedge w_{j}\left(s_{j}\right) & =-q^{-1} w_{j}\left(s_{j}\right) \wedge w_{i}\left(q^{-2} s_{i}\right), \\
w_{i}^{*}\left(s_{i}\right) \wedge w_{j}^{*}\left(s_{j}\right) & =-q w_{j}^{*}\left(s_{j}\right) \wedge w_{i}^{*}\left(q^{2} s_{i}\right)
\end{aligned}
$$

in $\Lambda(V)$ (respectively $\Lambda\left(V^{*}\right)$ ); hence, $\xi$ and $\xi_{\text {op }}$ (respectively $\xi^{*}$ and $\xi_{\text {op }}^{*}$ ) are related by the formulas

$$
\begin{aligned}
\xi\left(q^{2(n-1)} s_{1}, \ldots, q^{2} s_{n-1}, s_{n}\right) & =(-q)^{n(1-n) / 2} \xi_{\mathrm{op}}(\underline{s}), \\
\xi^{*}\left(q^{-2(n-1)} s_{1}, \ldots, q^{-2} s_{n-1}, s_{n}\right) & =(-q)^{n(n-1) / 2} \xi_{\mathrm{op}}^{*}(\underline{s}) .
\end{aligned}
$$

The norms of such vectors can be evaluated explicitly by an easy induction argument. The result is

$$
\begin{aligned}
\langle\xi(\underline{s}), \xi(\underline{t})\rangle & =\prod_{i=1}^{n}\left(1+s_{i}^{-1} t_{i}^{-1} q^{2(n-i)}\right), \\
\left\langle\xi^{*}(\underline{s}), \xi^{*}(\underline{t})\right\rangle_{*} & =q^{n(n+1)} \prod_{i=1}^{n}\left(1+s_{i}^{-1} t_{i}^{-1} q^{2(i-n)}\right) .
\end{aligned}
$$

We use the abbreviation $\xi(t)=\xi\left(\left(t^{n}\right)\right)$ for $t \in \mathbb{R} \backslash\{0\}$, where $\left(t^{n}\right)$ is the $n$-tuple with $t$ in each entry. Similarly we write $\xi_{\text {op }}(t), \xi^{*}(t)$ and $\xi_{\text {op }}^{*}(t)$. 


\section{SPHERICAL FUnCTIONS AND MACDONALD-KOORNWINDER POLYNOMIALS}

LEMMA 5.12.

(i) Let $\underline{s} \in(\mathbb{R} \backslash\{0\})^{n}$. Then $\operatorname{span}_{\mathbb{C}}\left\{\xi_{\text {op }}(\underline{s})\right\} \subset \Lambda^{n}(V)$ and $\operatorname{span}_{\mathbb{C}}\left\{\xi_{\text {op }}^{*}(\underline{s})\right\} \subset \Lambda^{n}\left(V^{*}\right)$ are one-dimensional $\mathcal{E}$-submodules if and only if $\underline{s}=\left(t^{n}\right)$ for some $t \in \mathbb{R} \backslash\{0\}$.

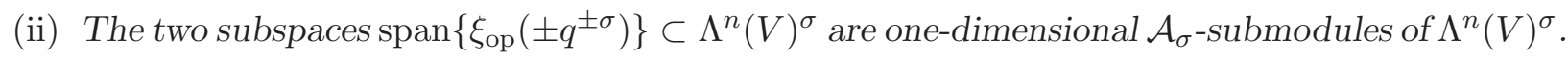
The generator $B_{n}^{\sigma} \in \mathcal{A}_{\sigma}$ acts by

$$
B_{n}^{\sigma} \xi_{\mathrm{op}}\left( \pm q^{ \pm \sigma}\right)=\vartheta_{1}\left( \pm q^{ \pm \sigma}\right) \xi_{\mathrm{op}}\left( \pm q^{ \pm \sigma}\right) .
$$

(iii) The two subspaces $\operatorname{span}\left\{\xi_{\mathrm{op}}^{*}\left( \pm q^{ \pm \sigma}\right)\right\} \subset \Lambda^{n}\left(V^{*}\right)^{\sigma}$ are one-dimensional $\mathcal{A}_{\sigma}$-submodules of $\Lambda^{n}\left(V^{*}\right)^{\sigma}$. The generator $B_{n}^{\sigma} \in \mathcal{A}_{\sigma}$ acts by

$$
B_{n}^{\sigma} \xi_{\text {op }}^{*}\left( \pm q^{ \pm \sigma}\right)=\vartheta_{-1}\left( \pm q^{ \pm \sigma}\right) \xi_{\text {op }}^{*}\left( \pm q^{ \pm \sigma}\right)
$$

Proof. (i) Fix $i \in\{1, \ldots, n-1\}$. By (5.11) we have

$$
x_{2 n-i} w_{j}(s)=y_{i} w_{j}(s)=0, \quad \forall j \in\{1, \ldots, n\} \backslash\{i\} .
$$

Combined with (5.12) and Proposition 4.2 we obtain

$$
\begin{aligned}
B_{i} \xi(\underline{s}) & =w_{1}\left(s_{1}\right) \wedge \cdots \wedge w_{i-1}\left(s_{i-1}\right) \wedge B_{i} w_{i}\left(s_{i}\right) \wedge K_{i}^{-1} K_{2 n-i}^{-1}\left(w_{i+1}\left(s_{i+1}\right) \wedge \cdots \wedge w_{n}\left(s_{n}\right)\right) \\
& =w_{1}\left(s_{1}\right) \wedge \cdots \wedge w_{i-1}\left(s_{i-1}\right) \wedge w_{i+1}\left(s_{i}\right) \wedge w_{i+1}\left(q s_{i+1}\right) \wedge w_{i+2}\left(s_{i+2}\right) \wedge \cdots \wedge w_{n}\left(s_{n}\right) .
\end{aligned}
$$

Since

$$
w_{i+1}\left(s_{i}\right) \wedge w_{i+1}\left(q s_{i+1}\right)=\left(s_{i}^{-1}-q^{-2} s_{i+1}^{-1}\right) v_{2 n-i} \wedge v_{i+1}
$$

in $\Lambda(V)$, we see that $B_{i} \xi(\underline{s})=0$ for all $i=1, \ldots, n-1$ if and only if $\underline{s}=\left(t q^{2(n-1)}, \ldots, t q^{2}, t\right)$ for some $t \in \mathbb{R} \backslash\{0\}$. Furthermore, $C_{j} \xi(\underline{s})=q \xi(\underline{s})$ for $j=1, \ldots, n$ by (5.12). Then Lemma 1.8 and (5.15) imply that $\xi_{\text {op }}(\underline{s})$ is a lowest $\mathcal{E} \simeq U_{q}(\mathfrak{g l}(n))$-weight vector of weight $\left(1^{n}\right)$ in $\Lambda^{n}(V)$ if and only if $\underline{s}=\left(t^{n}\right)$ for some $t \in \mathbb{R} \backslash\{0\}$, which implies (i) for $\xi_{\text {op }}$. The proof for $\xi_{\text {op }}^{*}$ is similar.

For the proof of (ii) and (iii), we note that Lemma 5.10(ii), (5.12) and (5.13) imply

$$
\begin{aligned}
B_{n}^{\sigma} \xi(\underline{s}) & =w_{1}\left(s_{1}\right) \wedge w_{2}\left(s_{2}\right) \wedge \cdots \wedge w_{n-1}\left(s_{n-1}\right) \wedge B_{n}^{\sigma} w_{n}\left(s_{n}\right), \\
B_{n}^{\sigma} \xi^{*}(\underline{s}) & =w_{1}^{*}\left(s_{1}\right) \wedge w_{2}^{*}\left(s_{2}\right) \wedge \cdots \wedge w_{n-1}^{*}\left(s_{n-1}\right) \wedge B_{n}^{\sigma} w_{n}^{*}\left(s_{n}\right) .
\end{aligned}
$$

Formula (5.14) now completes the proof.

In the following proposition we consider the $U_{q}(\mathfrak{g})$-module $\Lambda^{n}(V) \otimes \Lambda^{n}\left(V^{*}\right)$. By the Pieri formula (cf. [DS99]) its decomposition in irreducibles is given by

$$
\Lambda^{n}(V) \otimes \Lambda^{n}\left(V^{*}\right) \simeq \bigoplus_{m=0}^{n} L_{\omega_{m}^{\natural}},
$$

where $\omega_{m}=\left(1^{m}, 0^{n-m}\right) \in \Lambda_{n}^{+}$. We denote by $\pi_{n}: \Lambda^{n}(V) \otimes \Lambda^{n}\left(V^{*}\right) \rightarrow L_{\omega_{n}^{\natural}}$ the projection onto $L_{\omega_{n}^{\natural}}$ along the decomposition (5.17).

Since $\delta\left(0, \kappa_{1}\right)=\kappa_{1} \omega_{n},(5.17)$ and our earlier analysis of branching rules easily imply that the $V( \pm 1)_{\sigma}$-isotypical component of $\left(\Lambda^{n}(V) \otimes \Lambda^{n}\left(V^{*}\right)\right)^{\sigma}$ is one-dimensional (and necessarily lies in the component $L_{\omega_{n}^{\natural}}$, while the $V(0)_{\sigma^{-}}$-isotypical component of $\left(\Lambda^{n}(V) \otimes \Lambda^{n}\left(V^{*}\right)\right)^{\sigma}$ is $(n+1)$-dimensional. In [DS99], the $V(0)_{\sigma}$-isotypical component was constructed using the explicit solution $J^{\sigma}$ of the reflection equation. In the following proposition we give an explicit construction of one particular vector in the $V(0)_{\sigma}$-isotypical component and we explicitly construct the $V( \pm 1)_{\sigma}$-isotypical components of $\left(\Lambda^{n}(V) \otimes \Lambda^{n}\left(V^{*}\right)\right)^{\sigma}$. 


\section{A. A. Oblomkov and J. V. Stokman}

Proposition 5.13. Define $\eta_{k}(s) \in \Lambda^{n}(V) \otimes \Lambda^{n}\left(V^{*}\right)(s \in \mathbb{R} \backslash\{0\}, k \in\{-1,0,1\})$ by the formulas

$$
\begin{aligned}
\eta_{-1}(s) & =\xi_{\mathrm{op}}(s) \otimes \xi_{\mathrm{op}}^{*}\left(-q s^{-1}\right), \\
\eta_{0}(s) & =\xi_{\mathrm{op}}(s) \otimes \xi_{\mathrm{op}}^{*}\left(q^{-1} s\right), \\
\eta_{1}(s) & =\xi_{\mathrm{op}}\left(-s^{-1}\right) \otimes \xi_{\mathrm{op}}^{*}(q s) .
\end{aligned}
$$

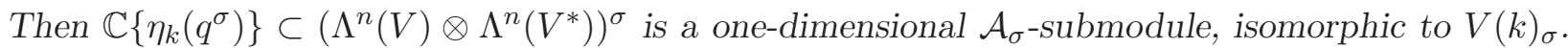
Furthermore, $\mathbb{C}\left\{\eta_{k}\left(q^{\sigma}\right)\right\} \quad(k= \pm 1)$ and $\pi_{n}\left(\mathbb{C}\left\{\eta_{0}\left(q^{\sigma}\right)\right\}\right)$ are realizations of the one-dimensional $\mathcal{A}_{\sigma}$-modules $V(k)_{\sigma}(k= \pm 1)$ and $V(0)_{\sigma}$ in $L_{\omega_{n}^{\natural}}^{\sigma}$, respectively.

Proof. By Proposition 4.2 and the previous lemma, $\mathbb{C}\left\{\xi_{\text {op }}(s) \otimes \xi_{\text {op }}^{*}(t)\right\} \subset \Lambda^{n}(V) \otimes \Lambda^{n}\left(V^{*}\right)$ is a copy of the trivial $\mathcal{E} \simeq U_{q}(\mathfrak{g l}(n))$ module for all $s, t \in \mathbb{R} \backslash\{0\}$. Furthermore, by Lemma 5.10 and the previous lemma,

$$
B_{n}^{\sigma}\left(\xi_{\mathrm{op}}\left( \pm q^{ \pm \sigma}\right) \otimes \xi_{\mathrm{op}}^{*}(t)\right)=\xi_{\mathrm{op}}\left( \pm q^{ \pm \sigma}\right) \otimes q^{-1} B_{n}^{\sigma \mp 1}\left(\xi_{\mathrm{op}}^{*}(t)\right)
$$

in $\Lambda^{n}(V) \otimes \Lambda^{n}\left(V^{*}\right)$ for all $t \in \mathbb{R} \backslash\{0\}$. The first statement now follows from Lemma 5.12 and the equalities

$$
q^{-1} \vartheta_{-1}\left(-q^{-\sigma+1}\right)=\vartheta_{0}\left(q^{\sigma-2}\right), \quad q^{-1} \vartheta_{-1}\left(q^{\sigma-1}\right)=\vartheta_{0}\left(q^{\sigma}\right), \quad q^{-1} \vartheta_{-1}\left(q^{\sigma+1}\right)=\vartheta_{0}\left(q^{\sigma+2}\right),
$$

which are the scalars by which $B_{n}^{\sigma}$ acts on $V(-1)_{\sigma}, V(0)_{\sigma}$ and $V(1)_{\sigma}$, respectively.

We have already observed that $\mathbb{C}\left\{\eta_{ \pm 1}\left(q^{\sigma}\right)\right\}$ automatically lies in the component $L_{\omega_{n}^{\natural}}^{\sigma}$ of the decomposition (5.17). In the decomposition of $\eta_{0}\left(q^{\sigma}\right)$ as the sum of basis elements $v_{I} \otimes v_{J}^{*}(\# I=$ $\# J=n)$, the coefficient of $v_{\{1, \ldots, n\}} \otimes v_{\{n+1, \ldots, 2 n\}}^{*}$ is nonvanishing, hence $\pi_{n}\left(\eta_{0}\left(q^{\sigma}\right)\right) \neq 0$. This completes the proof of the second statement of the proposition.

We define a $*$-unitary scalar product on $\Lambda^{n}(V) \otimes \Lambda^{n}\left(V^{*}\right)$ by

$$
\left\langle v \otimes v^{*}, w \otimes w^{*}\right\rangle_{1}=\langle v, w\rangle\left\langle v^{*}, w^{*}\right\rangle_{*} .
$$

The previous proposition implies that

$$
f_{k}^{\sigma, \tau}(\cdot)=\left\langle\cdot \pi_{n}\left(\eta_{k}\left(q^{\sigma}\right)\right), \eta_{1}\left(q^{\tau}\right)\right\rangle_{1}=\left\langle\cdot \eta_{k}\left(q^{\sigma}\right), \eta_{1}\left(q^{\tau}\right)\right\rangle_{1} \in F_{(1, k, 0)}^{\sigma, \tau}(0),
$$

for $k=-1,0,1$, are ground states. Their radial parts $\left.f_{k}^{\sigma, \tau}\right|_{T} \in \mathbb{C}\left[u^{ \pm 1}\right]$ can now be explicitly evaluated as follows.

Lemma 5.14. After suitable normalization, we have

$$
\begin{aligned}
& \left.f_{-1}^{\sigma, \tau}\right|_{T}=\prod_{i=1}^{n}\left(1-q^{1-\sigma+\tau} u_{i}^{-1}\right)\left(1-q^{-3+\sigma-\tau} u_{i}\right), \\
& \left.f_{0}^{\sigma, \tau}\right|_{T}=\prod_{i=1}^{n}\left(1-q^{1-\sigma+\tau} u_{i}^{-1}\right)\left(1+q^{-1-\sigma-\tau} u_{i}\right), \\
& \left.f_{1}^{\sigma, \tau}\right|_{T}=\prod_{i=1}^{n}\left(1+q^{1+\sigma+\tau} u_{i}^{-1}\right)\left(1+q^{-3-\sigma-\tau} u_{i}\right)
\end{aligned}
$$

in $\mathbb{C}\left[u^{ \pm 1}\right]$.

Proof. For $\lambda \in P_{2 n}$, we write $|\lambda|_{n}=\lambda_{1}+\lambda_{2}+\cdots+\lambda_{n}$. By (5.15) we have

$$
\begin{aligned}
& K^{\lambda-\delta} \xi_{\mathrm{op}}(s)=(-1)^{n(n-1) / 2} q^{\left.|\lambda|\right|_{n}-n^{2}} \xi\left(s q^{\lambda_{1}-\lambda_{2 n}-1}, s q^{\lambda_{2}-\lambda_{2 n-1}-1}, \ldots, s q^{\lambda_{n}-\lambda_{n+1}-1}\right), \\
& K^{\lambda-\delta} \xi_{\mathrm{op}}^{*}(s)=(-1)^{n(n-1) / 2} q^{-|\lambda|_{n}+n^{2}} \xi^{*}\left(s q^{1-\lambda_{1}+\lambda_{2 n}}, s q^{1-\lambda_{2}+\lambda_{2 n-1}}, \ldots, s q^{1-\lambda_{n}+\lambda_{n+1}}\right)
\end{aligned}
$$




\section{SPHERICAL FUnCTIONS AND MACDONALD-KOORNWINDER POLYNOMIALS}

for all $\lambda \in P_{2 n}$. By (5.15) and (5.16), we conclude that

$$
\begin{gathered}
\left\langle K^{\lambda-\delta} \xi_{\mathrm{op}}(s), \xi_{\mathrm{op}}(t)\right\rangle=q^{|\lambda|_{n}-n(n+1) / 2} \prod_{i=1}^{n}\left(1+s^{-1} t^{-1} q^{1+\lambda_{2 n+1-i}-\lambda_{i}}\right), \\
\left\langle K^{\lambda-\delta} \xi_{\mathrm{op}}^{*}(s), \xi_{\mathrm{op}}^{*}(t)\right\rangle_{*}=q^{-|\lambda|_{n}+3 n(n+1) / 2} \prod_{i=1}^{n}\left(1+s^{-1} t^{-1} q^{-1+\lambda_{i}-\lambda_{2 n+1-i}}\right)
\end{gathered}
$$

for all $\lambda \in P_{2 n}$. The lemma now follows easily from the definition of the $\eta_{k}$.

Lemma 5.15. Let $f \in F_{\left(\kappa_{1}, \kappa_{2}, 0\right)}^{\sigma, \tau}(0)$ be a ground state. After a suitable renormalization of $f$, we have

$$
\left.f\right|_{T}=u^{\delta\left(0, \kappa_{1}\right)} \prod_{i=1}^{n}\left(-q^{1+\sigma+\tau} u_{i}^{-1} ; q^{2}\right)_{\kappa_{1}+\kappa_{2}}\left(q^{1-\sigma+\tau} u_{i}^{-1} ; q^{2}\right)_{\kappa_{1}-\kappa_{2}}
$$

in $\mathbb{C}\left[u^{ \pm 1}\right]$.

Proof. We consider the $U_{q}(\mathfrak{g})$-module $W=\left(\Lambda^{n}(V) \otimes \Lambda^{n}\left(V^{*}\right)\right)^{\otimes \kappa_{1}}$, with $*$-unitary inner product

$$
\left\langle v_{1} \otimes \cdots \otimes v_{\kappa_{1}}, w_{1} \otimes \cdots \otimes w_{\kappa_{1}}\right\rangle_{\kappa_{1}}=\prod_{i=1}^{\kappa_{1}}\left\langle v_{i}, w_{i}\right\rangle_{1}
$$

for all $v_{i}, w_{j} \in \Lambda^{n}(V) \otimes \Lambda^{n}\left(V^{*}\right)$. Lemma 5.10 implies that the nonzero vector

$$
\chi_{\tau}=\eta_{1}\left(q^{\tau}\right) \otimes \eta_{1}\left(q^{\tau+2}\right) \otimes \cdots \otimes \eta_{1}\left(q^{\tau+2\left(\kappa_{1}-1\right)}\right) \in W
$$

spans a copy of the one-dimensional $\mathcal{A}_{\tau}$-module $V\left(\kappa_{1}\right)_{\tau}$ within $W^{\tau}$. By $(5.17)$ we have

$$
W \simeq L_{\delta\left(0, \kappa_{1}\right)^{\natural}} \oplus \bigoplus_{\lambda \in P_{2 n}^{+}: \lambda \prec \delta\left(0, \kappa_{1}\right)^{\natural}} L_{\lambda}^{\oplus d_{\lambda}}
$$

as $U_{q}(\mathfrak{g})$-modules for certain multiplicities $d_{\lambda} \in \mathbb{Z}_{\geqslant 0}$. By Proposition 1.15, $\mathbb{C}\left\{\chi_{\tau}\right\}$ lies in the component $L_{\delta\left(0, \kappa_{1}\right)^{\natural}}$ of the decomposition (5.18).

For the realization of $V\left(\kappa_{2}\right)_{\sigma}$ in $W^{\sigma}$, we need to consider the cases $0 \leqslant \kappa_{2} \leqslant \kappa_{1}$ and $-\kappa_{1} \leqslant \kappa_{2} \leqslant 0$ separately. For $0 \leqslant \kappa_{2} \leqslant \kappa_{1}$, Lemma 5.10 implies that the nonzero vector

$$
\chi_{\sigma}^{+}=\eta_{0}\left(q^{\sigma}\right)^{\otimes\left(\kappa_{1}-\kappa_{2}\right)} \otimes \eta_{1}\left(q^{\sigma}\right) \otimes \eta_{1}\left(q^{\sigma+2}\right) \otimes \cdots \otimes \eta_{1}\left(q^{\sigma+2\left(\kappa_{2}-1\right)}\right) \in W
$$

spans a copy of the one-dimensional $\mathcal{A}_{\sigma}$-module $V\left(\kappa_{2}\right)_{\sigma}$ in $W^{\sigma}$. If $p: W \rightarrow L_{\delta\left(0, \kappa_{1}\right)^{\natural}}$ denotes the projection on $L_{\delta\left(0, \kappa_{1}\right)^{\natural}}$ along the decomposition (5.18), then $p\left(\mathbb{C}\left\{\chi_{\sigma}^{+}\right\}\right)$is a copy of the one-dimensional $\mathcal{A}_{\sigma}$-module $V\left(\kappa_{2}\right)_{\sigma}$ in $L_{\delta\left(0, \kappa_{1}\right)^{\natural}}^{\sigma}$ by highest weight considerations, cf. Lemma 2.5. Thus

$$
f(\cdot)=\left\langle\cdot p\left(\chi_{\sigma}^{+}\right), \chi_{\tau}\right\rangle_{\kappa_{1}}=\left\langle\cdot \chi_{\sigma}^{+}, \chi_{\tau}\right\rangle_{\kappa_{1}}
$$

defines a ground state $f \in F_{\left(\kappa, \kappa_{2}, 0\right)}^{\sigma, \tau}(0)$. Since

$$
\left.f\right|_{T}=\left.\left.\prod_{i=1}^{\kappa_{1}-\kappa_{2}} f_{0}^{\sigma, \tau+2(i-1)}\right|_{T} \prod_{j=1}^{\kappa_{2}} f_{1}^{\sigma+2(j-1), \tau+2\left(\kappa_{1}-\kappa_{2}+j-1\right)}\right|_{T},
$$

the result now follows from the previous lemma by a straightforward manipulation of $q$-shifted factorials. For $-\kappa_{1} \leqslant \kappa_{2} \leqslant 0$ the vector $\chi_{\sigma}^{+}$should be replaced by

$$
\chi_{\sigma}^{-}=\eta_{0}\left(q^{\sigma}\right)^{\otimes\left(\kappa_{1}+\kappa_{2}\right)} \otimes \eta_{-1}\left(q^{\sigma}\right) \otimes \eta_{-1}\left(q^{\sigma-2}\right) \otimes \cdots \otimes \eta_{-1}\left(q^{\sigma-2\left(-\kappa_{2}-1\right)}\right) \in W .
$$

We leave the remaining straightforward computations to the reader. 


\section{A. A. Oblomkov and J. V. Stokman}

Theorem 1.21(ii) now follows immediately by combining Lemma 5.1, Corollary 5.9 and Lemma 5.15.

\section{Generalized quantum Schur orthogonality relations}

We freely use the notation of the previous sections. The normalized Haar functional $h: \mathbb{C}_{q}[G] \rightarrow \mathbb{C}$ is the unique linear map which intertwines the (left) regular $U_{q}(\mathfrak{g})$-action on $\mathbb{C}_{q}[G]$ with the trivial $U_{q}(\mathfrak{g})$-action on $\mathbb{C}$ and which maps $1 \in \mathbb{C}_{q}[G]$ to $1 \in \mathbb{C}$. In particular, $h$ is identically zero on the summands $W(\lambda)\left(\lambda \in P_{2 n}^{+} \backslash\{0\}\right)$ in the Peter-Weyl decomposition (1.18).

The Haar functional $h$ defines a pre-Hilbert space structure on $\mathbb{C}_{q}[G]$ by

$$
\langle a, b\rangle_{h}=h\left(b^{*} a\right), \quad \forall a, b \in \mathbb{C}_{q}[G] .
$$

The quantum Schur orthogonality relations imply that the Peter-Weyl decomposition (1.18) is an orthogonal decomposition with respect to $\langle\cdot, \cdot\rangle_{h}$.

We fix a scalar product $\langle\cdot, \cdot\rangle_{\vec{\kappa}}$ on the simple $U_{q}(\mathfrak{k})$-module $V\left(\kappa, \kappa_{1}\right)$ such that the representation map $\rho\left(\kappa, \kappa_{1}\right)$ is $*$-unitary (of course, $\langle\cdot, \cdot\rangle_{\vec{\kappa}}$ only depends on the parameters $\kappa, \kappa_{1}$ of $\vec{\kappa}$, and is unique up to scalar multiples). From Corollary 1.11 it follows that the simple $\mathcal{A}_{\tau}$-module $\left(\rho\left(\kappa, \kappa_{1}\right)_{\tau}, V\left(\kappa, \kappa_{1}\right)_{\tau}\right)$ is $*$-unitary with respect to $\langle\cdot, \cdot\rangle_{\vec{\kappa}}$.

Definition 6.1. Let $\pi=\pi_{\vec{\kappa}}^{\sigma, \tau}: F_{\vec{\kappa}}^{\sigma, \tau} \times F_{\vec{\kappa}}^{\sigma, \tau} \rightarrow \operatorname{Hom}\left(U_{q}(\mathfrak{g}), \mathbb{C}\right)$ be the sesquilinear form

$$
\pi(f, g)(X)=\sum\left\langle f\left(K^{-\delta} X_{1}\right), g\left(K^{-\delta} \omega\left(X_{2}\right)\right)\right\rangle_{\vec{\kappa}}, \quad f, g \in F_{\vec{\kappa}}^{\sigma, \tau}, X \in U_{q}(\mathfrak{g}) .
$$

Let $f, g \in F_{\vec{\kappa}}^{\sigma, \tau}$. We write $f \cdot K^{-\delta}$ for the right regular action of $K^{-\delta} \in U_{q}(\mathfrak{g})$ on the first tensor component of $f \in \mathbb{C}_{q}[G] \otimes V\left(\kappa, \kappa_{1}\right)_{\tau}$, and similarly for $g \cdot K^{-\delta}$. If

$$
f \cdot K^{-\delta}=\sum f_{i} \otimes v_{i}, \quad g \cdot K^{-\delta}=\sum g_{j} \otimes w_{j},
$$

with $f_{i}, g_{j} \in \mathbb{C}_{q}[G]$ and $v_{i}, w_{j} \in V\left(\kappa, \kappa_{1}\right)_{\tau}$, then the definition of the pairing $\pi$ and (3.2) imply that

$$
\pi(f, g)(X)=\sum f_{i}\left(X_{1}\right) g_{j}^{*}\left(X_{2}\right)\left\langle v_{i}, w_{j}\right\rangle_{\vec{\kappa}}=\sum\left(f_{i} g_{j}^{*}\right)(X)\left\langle v_{i}, w_{j}\right\rangle_{\vec{\kappa}}
$$

for all $X \in U_{q}(\mathfrak{g})$. In particular, the image of $\pi$ is contained in $\mathbb{C}_{q}[G]$. This observation can be strengthened as follows.

Proposition 6.2. The image of the pairing $\pi_{\vec{\kappa}}^{\sigma, \tau}$ is contained in $\mathcal{H}^{\sigma, \tau}$.

Proof. We use the characterization of the space $\mathcal{H}^{\sigma, \tau}$ as given in Remark 3.9(ii).

Fix $f, g \in F_{\vec{\kappa}}^{\sigma, \tau}$. Let $a \in \mathcal{A}_{\sigma}$ and write $\Delta(a)=\sum a_{1} \otimes a_{2}$ with the $a_{1}$ from $\mathcal{A}_{\sigma}$ (which can be done in view of Proposition 4.2). Using the fact that the character $\chi\left(\kappa_{2}\right)_{\sigma}: \mathcal{A}_{\sigma} \rightarrow \mathbb{C}$ of $V\left(\kappa_{2}\right)_{\sigma}$ is *-unitary, $\chi\left(\kappa_{2}\right)_{\sigma}\left(a^{*}\right)=\overline{\chi\left(\kappa_{2}\right)_{\sigma}(a)}$ for $a \in \mathcal{A}_{\sigma}$, we obtain for $X \in U_{q}(\mathfrak{g})$,

$$
\begin{aligned}
\pi(f, g)(X a) & =\sum \chi\left(\kappa_{2}\right)_{\sigma}\left(a_{1}\right)\left\langle f\left(K^{-\delta} X_{1}\right), g\left(K^{-\delta} \omega\left(X_{2}\right) \omega\left(a_{2}\right)\right)\right\rangle_{\vec{\kappa}} \\
& =\sum\left\langle f\left(K^{-\delta} X_{1}\right), g\left(K^{-\delta} \omega\left(X_{2}\right) \omega\left(a_{2}\right) a_{1}^{*}\right)\right\rangle_{\vec{\kappa}} \\
& =\epsilon(a) \pi(f, g)(X)
\end{aligned}
$$

since

$$
\sum \omega\left(a_{2}\right) a_{1}^{*}=\left(\sum a_{1} S\left(a_{2}\right)\right)^{*}=\overline{\epsilon(a)} 1
$$

by the antipode axiom for the Hopf algebra $U_{q}(\mathfrak{g})$. For the left invariance with respect to $\mathcal{A}_{\tau}$, let $b \in \mathcal{A}_{\tau}$, and again write $\Delta(b)=\sum b_{1} \otimes b_{2}$ with all $b_{1}$ from $\mathcal{A}_{\tau}$. Now $\Delta$ is a $*$-algebra homomorphism 


\section{SPHERICAL FUNCTIONS AND MACDONALD-KOORNWINDER POLYNOMIALS}

and $S$ is an anti-coalgebra homomorphism, so

$$
\Delta\left(\omega_{\delta}(b)\right)=\sum \omega_{\delta}\left(b_{2}\right) \otimes \omega_{\delta}\left(b_{1}\right) .
$$

Furthermore, note that $\left(\omega \circ \omega_{\delta}\right)(X)=K^{\delta} X K^{-\delta}$ for $X \in U_{q}(\mathfrak{g})$. Combined with (3.6), we thus obtain for $X \in U_{q}(\mathfrak{g})$,

$$
\begin{aligned}
\pi(f, g)\left(\omega_{\delta}(b) X\right) & =\sum\left\langle f\left(K^{-\delta} \omega_{\delta}\left(b_{2}\right) X_{1}\right), g\left(b_{1} K^{-\delta} \omega\left(X_{2}\right)\right)\right\rangle_{\vec{\kappa}} \\
& =\sum\left\langle f\left(K^{-2 \delta} \omega\left(b_{2}\right) K^{\delta} X_{1}\right), \rho\left(\kappa, \kappa_{1}\right)_{\tau}\left(b_{1}\right) g\left(K^{-\delta} \omega\left(X_{2}\right)\right)\right\rangle_{\vec{\kappa}} \\
& =\sum\left\langle f\left(b_{1}^{*} S^{2}\left(\omega\left(b_{2}\right)\right) K^{-\delta} X_{1}\right), g\left(K^{-\delta} \omega\left(X_{2}\right)\right)\right\rangle_{\vec{\kappa}} \\
& =\overline{\epsilon(b)} \pi(f, g)(X),
\end{aligned}
$$

since

$$
\sum b_{1}^{*} S^{2}\left(\omega\left(b_{2}\right)\right)=\sum b_{1}^{*} S\left(b_{2}^{*}\right)=\epsilon\left(b^{*}\right) 1=\overline{\epsilon(b)} 1 .
$$

Choose a vector $v \in \widetilde{V}\left(\kappa, \kappa_{1}\right)_{\tau}$ such that $\langle v, v\rangle_{\vec{\kappa}}=1$. Without loss of generality we may and will assume that the implicit identification $\widetilde{V}\left(\kappa, \kappa_{1}\right)_{\tau} \simeq \mathbb{C}$ in the definition of the restriction map $\left.\right|_{T}$ is realized by the explicit map $z v \mapsto z(z \in \mathbb{C})$.

Corollary 6.3. If $f, g \in F_{\vec{\kappa}}^{\sigma, \tau}$, then $\left.f\right|_{T}\left(\left.g\right|_{T}\right)^{*} \in \mathbb{C}\left[u^{ \pm 1}\right]^{W}$.

Proof. Let $f, g \in F_{\vec{\kappa}}^{\sigma, \tau}$. Since the Cartan elements $K^{\lambda}\left(\lambda \in P_{2 n}\right)$ are group-like and $*$-self-adjoint elements in $U_{q}(\mathfrak{g})$, it follows from Lemma 1.20 and from the definition of $\pi$ that

$$
\begin{aligned}
\operatorname{Res}_{T}(\pi(f, g))\left(q^{\lambda}\right) & =\pi(f, g)\left(K^{\lambda}\right) \\
& =\left\langle f\left(K^{\lambda-\delta}\right), g\left(K^{-\lambda-\delta}\right)\right\rangle_{\vec{\kappa}} \\
& =\left.f\right|_{T}\left(q^{\lambda}\right)\left(\left.g\right|_{T}\right)^{*}\left(q^{\lambda}\right)
\end{aligned}
$$

for $\lambda \in P_{2 n}$, and hence

$$
\operatorname{Res}_{T}(\pi(f, g))=\left.f\right|_{T}\left(\left.g\right|_{T}\right)^{*}
$$

in $\mathbb{C}\left[u^{ \pm 1}\right]$. On the other hand, $\operatorname{Res}_{T}(\pi(f, g)) \in \mathbb{C}\left[u^{ \pm 1}\right]^{W}$ by the previous proposition and by Proposition 3.10 .

We define a linear functional $h^{\sigma, \tau}: \mathbb{C}\left[u^{ \pm 1}\right] \rightarrow \mathbb{C}$ by integrating against the orthogonality measure of the Macdonald-Koornwinder polynomials $P_{\mu}^{\sigma, \tau}\left(\mu \in \Lambda_{n}^{+}\right)$, see (3.8). We renormalize $h^{\sigma, \tau}$ so that $h^{\sigma, \tau}(1)=1$. In particular, for real parameters $\sigma, \tau$ satisfying

$$
q^{\epsilon \sigma+\epsilon^{\prime} \tau}<q^{-1}, \quad \forall \epsilon, \epsilon^{\prime} \in\{ \pm 1\}
$$

the functional $h^{\sigma, \tau}$ is given by

$$
h^{\sigma, \tau}(p)=\frac{1}{N} \int_{T} p(u) \Delta_{\sigma, \tau}(u) \frac{d u}{u}, \quad p \in \mathbb{C}\left[u^{ \pm 1}\right],
$$

with $\Delta_{\sigma, \tau}(u)=\Delta\left(u ;-q^{\sigma+\tau+1},-q^{-\sigma-\tau+1}, q^{\sigma-\tau+1}, q^{-\sigma+\tau+1} ; q^{2}, q^{2}\right)$ the Macdonald-Koornwinder weight function and with positive normalization constant $N=\int_{T} \Delta_{\sigma, \tau}(u) d u$. Then $h^{\sigma, \tau}$ gives rise to a pre-Hilbert structure on $\mathbb{C}\left[u^{ \pm 1}\right]$,

$$
\langle p, r\rangle_{\sigma, \tau}=h^{\sigma, \tau}\left(r^{*} p\right), \quad p, r \in \mathbb{C}\left[u^{ \pm 1}\right]
$$

with $*$-structure on $\mathbb{C}\left[u^{ \pm 1}\right]$ defined by (3.7). Theorem 1.21 for $\vec{\kappa}=\overrightarrow{0}$ and the orthogonality relations for the Macdonald-Koornwinder polynomials $P_{\mu}^{\sigma, \tau}$ imply $h(f)=h^{\sigma, \tau}\left(\left.f\right|_{T}\right)$ for $f \in F_{\overrightarrow{0}}^{\sigma, \tau}$. By Proposition 3.8 this implies

$$
h(f)=h^{\sigma, \tau}\left(\operatorname{Res}_{T}(f)\right), \quad \forall f \in \mathcal{H}^{\sigma, \tau} .
$$




\section{A. A. Oblomkov and J. V. Stokman}

Since $\operatorname{Res}_{T}: \mathcal{H}^{\sigma, \tau} \rightarrow \mathbb{C}\left[u^{ \pm 1}\right]^{W}$ is a *-algebra isomorphism, we conclude from (6.3) that

$$
\operatorname{Res}_{T}:\left(\mathcal{H}^{\sigma, \tau},\langle\cdot, \cdot\rangle_{h}\right) \rightarrow\left(\mathbb{C}\left[u^{ \pm 1}\right]^{W},\langle\cdot, \cdot\rangle_{\sigma, \tau}\right)
$$

is an isomorphism of pre-Hilbert spaces.

Proposition 6.4 (Generalized quantum Schur orthogonality relations). Let $\mu, \nu \in \Lambda_{n}^{+}$and choose elementary vector valued spherical functions $f \in F_{\vec{\kappa}}^{\sigma, \tau}(\mu)$ and $g \in F_{\vec{\kappa}}^{\sigma, \tau}(\nu)$. If $\mu \neq \nu$, then $\left\langle\left. f\right|_{T},\left.g\right|_{T}\right\rangle_{\sigma, \tau}=0$.

Proof. Let $f, g \in F_{\vec{\kappa}}^{\sigma, \tau}$. By the proof of Corollary 6.3, by Proposition 6.2 and by (6.3) we have

$$
h(\pi(f, g))=\left\langle\left. f\right|_{T},\left.g\right|_{T}\right\rangle_{\sigma, \tau} .
$$

Let $f \in F_{\vec{\kappa}}^{\sigma, \tau}(\mu)$ and $g \in F_{\vec{\kappa}}^{\sigma, \tau}(\nu)$ with $\mu, \nu \in \Lambda_{n}^{+}$and $\mu \neq \nu$. We can show that the left-hand side of (6.4) vanishes. Since $f \cdot K^{-\delta} \in W\left(\mu^{\natural}\right) \otimes V\left(\kappa, \kappa_{1}\right)_{\tau}$ and $g \cdot K^{-\delta} \in W\left(\nu^{\natural}\right) \otimes V\left(\kappa, \kappa_{1}\right)_{\tau}$, we may write

$$
f \cdot K^{-\delta}=\sum f_{i} \otimes v_{i}, \quad g \cdot K^{-\delta}=\sum g_{j} \otimes w_{j},
$$

with $f_{i} \in W\left(\mu^{\natural}\right), g_{j} \in W\left(\nu^{\natural}\right)$ and $v_{i}, w_{j} \in V\left(\kappa, \kappa_{1}\right)_{\tau}$. By (6.1) and by the quantum Schur orthogonality relations, we conclude that

$$
h(\pi(f, g))=\sum h\left(f_{i} g_{j}^{*}\right)\left\langle v_{i}, w_{j}\right\rangle_{\vec{\kappa}}=0,
$$

as desired.

The main application of the generalized quantum Schur orthogonality relations is the identification of the vector valued spherical functions with Macdonald-Koornwinder polynomials, as stated in Theorem 1.21(iii).

Corollary 6.5. Let $\mu \in \Lambda_{n}^{+}$and choose elementary vector valued spherical functions $f_{\mu} \in F_{\vec{\kappa}}^{\sigma, \tau}(\mu)$ and $f_{0} \in F_{\vec{\kappa}}^{\sigma, \tau}(0)$. Then

$$
\frac{\left.f_{\mu}\right|_{T}}{\left.f_{0}\right|_{T}}=D P_{\mu}\left(u ;-q^{\sigma+\tau+1+\kappa_{1}+\kappa_{2}},-q^{-\sigma-\tau+1}, q^{\sigma-\tau+1}, q^{-\sigma+\tau+1+\kappa_{1}-\kappa_{2}} ; q^{2}, q^{2 \kappa+2}\right)
$$

for some nonzero constant $D$.

Proof. It suffices to prove the corollary for real parameters $\sigma, \tau$ satisfying (6.2). By Proposition 4.5 we can normalize the elementary vector valued spherical functions $f_{\mu} \in F_{\vec{\kappa}}^{\sigma, \tau}(\mu)\left(\mu \in \Lambda_{n}^{+}\right)$such that

$$
p_{\mu}:=\frac{\left.f_{\mu}\right|_{T}}{\left.f_{0}\right|_{T}}=m_{\mu}+\sum_{\substack{\mu^{\prime} \in \Lambda_{n}^{+} \\ \mu^{\prime}<\mu}} c_{\mu^{\prime}} m_{\mu^{\prime}} \in \mathbb{C}\left[u^{ \pm 1}\right]^{W}
$$

for some constants $c_{\mu^{\prime}} \in \mathbb{C}$. We now prove that $p_{\mu}$ is the monic Macdonald-Koornwinder polynomial of degree $\mu$ with suitable specialization of the parameters. By the generalized quantum Schur orthogonality relations we have

$$
\int_{T} p_{\mu}(u) p_{\nu}^{*}(u) W(u) \frac{d u}{u}=0, \quad \forall \nu \in \Lambda_{n}^{+}: \nu \neq \mu
$$

with weight function $W(u)=\left.f_{0}\right|_{T}(u)\left(\left.f_{0}\right|_{T}\right)^{*}(u) \Delta_{\sigma, \tau}(u)$. By Theorem 1.21(ii), the weight function $W(u)(u \in T)$ can be expressed in terms of the Macdonald-Koornwinder weight function $\Delta(u ; a, b, c, d ; q, t)$ as

$$
W(u)=C \Delta\left(u ;-q^{\sigma+\tau+1+\kappa_{1}+\kappa_{2}},-q^{-\sigma-\tau+1}, q^{\sigma-\tau+1}, q^{-\sigma+\tau+1+\kappa_{1}-\kappa_{2}} ; q^{2}, q^{2 \kappa+2}\right)
$$

for some nonzero constant $C$. Properties (6.5) and (6.6) thus imply

$$
\int_{T} p_{\mu}(u) m_{\nu}(u) W(u) \frac{d u}{u}=0, \quad \forall \nu \in \Lambda_{n}^{+}: \nu<\mu .
$$




\section{SPHERICAL FUnCTIONS AND MACDONALD-KOORNWINDER POLYNOMIALS}

The proof now follows from the fact that the Macdonald-Koornwinder polynomial

$$
P_{\mu}\left(u ;-q^{\sigma+\tau+1+\kappa_{1}+\kappa_{2}},-q^{-\sigma-\tau+1}, q^{\sigma-\tau+1}, q^{-\sigma+\tau+1+\kappa_{1}-\kappa_{2}} ; q^{2}, q^{2 \kappa+2}\right) \in \mathbb{C}\left[u^{ \pm 1}\right]^{W}
$$

has been defined as the unique $W$-invariant Laurent polynomial satisfying the properties (6.5) and (6.7).

\section{ACKNOWLEDGEMENTS}

A substantial part of the research was done when the second author visited Massachusetts Institute of Technology for a period of three months in the beginning of 2002 and when the first author visited the $\mathrm{KdV}$ institute in the summer of 2002. Both authors are grateful for the hospitality of the institutes. Both authors thank Pavel Etingof for stimulating discussions.

\section{REFERENCES}

Bab91 O. Babelon, Universal exchange algebra for Bloch waves and Liouville theory, Comm. Math. Phys. 139 (1991), 619-643.

DS99 M. S. Dijkhuizen and J. V. Stokman, Some limit transitions between BC type orthogonal polynomials interpreted on quantum complex Grassmannians, Publ. Res. Inst. Math. Sci. Kyoto Univ. 35 (1999), 451-500.

EK94 P. I. Etingof and A. A. Kirillov Jr., Macdonald's polynomials and representations of quantum groups, Math. Res. Lett. 1 (1994), 279-294.

EV00 P. I. Etingof and A. Varchenko, Traces of intertwiners for quantum groups and difference equations, I, Duke Math. J. 104 (2000), 391-432.

FR92 I. Frenkel and N. Reshetikhin, Quantum affine algebras and holonomic difference equations, Comm. Math. Phys. 146 (1992), 1-60.

Kas95 C. Kassel, Quantum groups, Graduate Texts in Mathematics, vol. 155 (Springer, Berlin, 1995).

Kir95 A. A. Kirillov Jr., Traces of intertwining operators and Macdonald polynomials, PhD thesis, Yale University (1995), q-alg/9503012.

Koe96 H. T. Koelink, Askey-Wilson polynomials and the quantum SU(2) group: survey and applications, Acta Appl. Math. 44 (1996), 295-352.

Koo93 T. H. Koornwinder, Askey-Wilson polynomials as zonal spherical functions on the SU(2) quantum group, SIAM J. Math. Anal. 24 (1993), 795-813.

Koo92 T. H. Koornwinder, Askey-Wilson polynomials for root systems of type BC, in Hypergeometric functions on domains of positivity, Jack polynomials, and applications, ed. D. S. P. Richards, Contemporary Mathematics, vol. 138 (American Mathematical Society, Providence, RI, 1992), 189-204.

Let97 G. Letzter, Subalgebras which appear in quantum Iwasawa decompositions, Canad. J. Math. 49 (1997), 1206-1223.

Let99 G. Letzter, Symmetric pairs for quantized enveloping algebras, J. Algebra 220 (1999), 729-767.

Let00 G. Letzter, Harish-Chandra modules for quantum symmetric pairs, Represent. Theory 4 (2000), 64-96.

Let02 G. Letzter, Coideal subalgebras and quantum symmetric pairs, in New directions in Hopf algebras, Math. Sci. Res. Inst. Publ., vol. 43 (Cambridge University Press, Cambridge, 2002), 117-165.

Let03 G. Letzter, Quantum symmetric pairs and their zonal spherical functions, Transform. Groups 8 (2003), 261-292.

Let04 G. Letzter, Quantum zonal spherical functions and Macdonald polynomials, Adv. Math. 189 (2004), 88-147.

Mac00 I. G. Macdonald, Orthogonal polynomials associated with root systems, Sém. Lothar. Combin. 45 (2000/01), Article B45a. 


\section{SPHERICAL FUnCTIONS AND MACDONALD-KOORNWINDER POLYNOMIALS}

Nou96 M. Noumi, Macdonald's symmetric polynomials as zonal spherical function on some quantum homogeneous spaces, Adv. Math. 123 (1996), 16-77.

NDS97 M. Noumi, M. S. Dijkhuizen and T. Sugitani, Multivariable Askey-Wilson polynomials and quantum complex Grassmanians, in Proc. workshop 'special functions, q-series and related topics', Toronto, June 19-23, 1995, Fields Inst. Comm., vol. 14 (American Mathematical Society, Providence, RI, 1997), 167-177.

NM90 M. Noumi and K. Mimachi, Askey-Wilson polynomials and the quantum group $S U_{q}(2)$, Proc. Japan Acad. Ser. A Math. Sci. 66 (1990), 146-149.

NYM93 M. Noumi, H. Yamada and K. Mimachi, Finite-dimensional representations of the quantum group $G L_{q}(n ; \mathbb{C})$ and the zonal spherical functions on $U_{q}(n-1) \backslash U_{q}(n)$, Japan. J. Math. (N.S.) 19 (1993), $31-80$.

Obl04 A. Oblomkov, Heckman-Opdam Jacobi polynomials for $B C_{n}$ root system and generalized spherical functions, Adv. Math. 186 (2004), 153-180.

Ros00 H. Rosengren, A new quantum algebraic interpretation of the Askey-Wilson polynomials, in q-series from a contemporary perspective, South Hadley, MA, 1998, Contemporary Mathematics, vol. 254 (American Mathematical Society, Providence, RI, 2000), 371-394.

Sto00 J. V. Stokman, On BC type basic hypergeometric orthogonal polynomials, Trans. Amer. Math. Soc. 352 (2000), 1527-1579.

Sto03 J. V. Stokman, Vertex-IRF transformations, dynamical quantum groups and harmonic analysis, Indag. Math. (N.S.) 14 (2003), 545-570.

Alexei A. Oblomkov oblomkov@math.mit.edu Department of Mathematics, Massachusetts Institute of Technology, 77 Massachusetts Avenue, Cambridge, MA 02139, USA

Jasper V. Stokman jstokman@science.uva.nl KdV Institute for Mathematics, Universiteit van Amsterdam, Plantage Muidergracht 24, 1018 TV Amsterdam, The Netherlands 